\title{
On the Kashaev invariant and the twisted Reidemeister torsion of two-bridge knots
}

\author{
TOMOTADA OHTSUKI \\ TOSHIE TAKATA
}

\begin{abstract}
It is conjectured that, in the asymptotic expansion of the Kashaev invariant of a hyperbolic knot, the first coefficient is represented by the complex volume of the knot complement, and the second coefficient is represented by a constant multiple of the square root of the twisted Reidemeister torsion associated with the holonomy representation of the hyperbolic structure of the knot complement. In particular, this conjecture has been rigorously proved for some simple hyperbolic knots, for which the second coefficient is presented by a modification of the square root of the Hessian of the potential function of the hyperbolic structure of the knot complement.

In this paper, we define an invariant of a parametrized knot diagram as a modification of the Hessian of the potential function obtained from the parametrized knot diagram. Further, we show that this invariant is equal (up to sign) to a constant multiple of the twisted Reidemeister torsion for any two-bridge knot.
\end{abstract}

$57 \mathrm{M} 27$

\section{Introduction}

In $[11 ; 12]$, Kashaev defined the Kashaev invariant $\langle L\rangle_{N} \in \mathbb{C}$ of a link $L$ for $N=2,3, \ldots$ by using the quantum dilogarithm at $q=e^{2 \pi \sqrt{-1} / N}$. In [13], he conjectured that, for any hyperbolic link $L, \frac{2 \pi}{N} \log \langle L\rangle_{N}$ goes to the hyperbolic volume of $S^{3}-L$ as $N \rightarrow \infty$ and verified the conjecture for some simple knots by formal calculations. In [15], H Murakami and J Murakami proved that the Kashaev invariant $\langle L\rangle_{N}$ of any link $L$ is equal to the $N$-colored Jones polynomial $J_{N}\left(L ; e^{2 \pi \sqrt{-1} / N}\right)$ of $L$ evaluated at $q=e^{2 \pi \sqrt{-1} / N}$. Further, as an extension of Kashaev's conjecture, they conjectured that for any knot $K, \frac{2 \pi}{N} \log \left|J_{N}\left(K ; e^{2 \pi \sqrt{-1} / N}\right)\right|$ goes to the (normalized) simplicial volume of $S^{3}-K$. This is called the volume conjecture. As a complexification of the volume conjecture, it is conjectured by H Murakami, J Murakami, Okamoto, Takata and Yokota [16] that for a hyperbolic link $L, J_{N}\left(L ; e^{2 \pi \sqrt{-1} / N}\right) \sim e^{N \varsigma(L)}$ as $N \rightarrow \infty$, where we set

$$
\varsigma(L)=\frac{1}{2 \pi \sqrt{-1}}\left(\operatorname{cs}\left(S^{3}-L\right)+\sqrt{-1} \operatorname{vol}\left(S^{3}-L\right)\right),
$$


and "cs" and "vol" denote the Chern-Simons invariant and the hyperbolic volume; we call it the complex hyperbolic volume (we use the $\mathrm{SL}_{2} \mathbb{C}$ Chern-Simons invariant). Furthermore, it was conjectured by Gukov [9] (see also Dimofte, Gukov, Lenells and Zagier [4], Gukov and H Murakami [10] and Zagier [33]) from the viewpoint of $\mathrm{SL}_{2} \mathbb{C}$ Chern-Simons theory that the asymptotic expansion of $J_{N}\left(K ; e^{2 \pi \sqrt{-1} / k}\right)$ of a hyperbolic knot $K$ is presented by the form

$$
J_{N}\left(K ; e^{2 \pi \sqrt{-1} / k}\right) \underset{\substack{N, k \rightarrow \infty \\ u=N / k \mid \text { fixed }}}{\sim} e^{N \varsigma} N^{3 / 2} \omega \cdot\left(1+\sum_{i=1}^{\infty} \kappa_{i} \cdot\left(\frac{2 \pi \sqrt{-1}}{N}\right)^{i}\right)
$$

as $N, k \rightarrow \infty$ for $u=N / k$ fixed and for some scalars $\varsigma, \omega, \kappa_{i}$ depending on $K$ and $u$. These authors do not discuss the Jones polynomial of Chern-Simons theory in the case of vanishing quantum dimension, which Witten does in [29]. We note that the colored Jones polynomial is defined at generic $q$, while the Kashaev invariant is defined only at $q=e^{2 \pi \sqrt{-1} / N}$. Andersen and Hansen [1] proved the semiclassical approximation (ie the " $e^{N \varsigma} N^{3 / 2} \omega$ " part) of the above expansion is proved for the figure-eight knot at $q=e^{2 \pi \sqrt{-1} / N}$ and $\mathrm{H}$ Murakami proved it in [14] at generic $q$ around $e^{2 \pi \sqrt{-1} / N}$. As for rigorous proofs for other hyperbolic knots, it is shown by the first author in [17; 18] and the first author and Yokota [20] that for any hyperbolic knot $K$ with up to 7 crossings, the asymptotic expansions of the Kashaev invariant of $K$ is represented by

$$
\langle K\rangle_{N}=e^{N \varsigma(K)} N^{3 / 2} \omega(K) \cdot\left(1+\sum_{i=1}^{d} \kappa_{i}(K) \cdot\left(\frac{2 \pi \sqrt{-1}}{N}\right)^{i}+O\left(\frac{1}{N^{d+1}}\right)\right),
$$

for any $d$, where the $\omega(K)$ and $\kappa_{i}(K)$ are some scalars. In another approach to this problem, Dimofte and Garoufalidis [3], motivated by the above mentioned conjectures, constructed a formal power series as an invariant of a hyperbolic knot by using the canonical simplicial decomposition of the hyperbolic knot complement; it is conjectured that this power series is equal to the expansion (2).

We consider the second coefficient of the semiclassical approximation (ie the " $\omega$ " part) of the above expansions. As explained in Witten [28], such a coefficient of the semiclassical approximation of the Chern-Simons path integral is calculated as the regularized determinant of the Laplacian, and it is represented by the square root of the Ray-Singer torsion at a flat connection, which is equal to the twisted Reidemeister torsion. Further, by similar arguments, it is conjectured in $[9 ; 10 ; 14]$ that the $\omega$ of (1) is a scalar multiple of the square root of (the Ray-Singer torsion at a flat connection or) the twisted Reidemeister torsion of the cochain complex of the knot complement with the $\mathfrak{s l}_{2} \mathbb{C}$ coefficient twisted by the adjoint action of the holonomy representation of the hyperbolic structure of the knot complement; this conjecture is confirmed for 
the figure-eight knot in [1;14], and numerically checked for some knots in Dubois [5]. Furthermore, the " $\omega$ " part of the power series of [3] is conjectured (and confirmed in many cases) to be a constant multiple of the square root of the twisted Reidemeister torsion. Hence we conjecture that $\omega(K)$ of (2) is equal to a constant multiple of the square root of the twisted Reidemeister torsion. In the proof of $(2)$ in $[17 ; 18 ; 20]$, we use the Poisson summation formula and the saddle point method (see Section 4.2 and $[17 ; 18 ; 20])$, and we must check many technical concrete inequalities to calculate such procedures. Because of such technical difficulties, it is difficult at the present stage to prove (2) rigorously for general knots. However, by assuming the inequalities from the saddle point method hold, we can guess the resulting form of (2). In particular, by a formal calculation based on such assumptions, $\omega(K)^{-2}$ is represented by a modification of the Hessian of the potential function obtained from a knot diagram parameterized by hyperbolicity parameters.

In this paper, we formulate $\omega_{2}(D)$ of a parameterized diagram $D$ of a knot $K$ such that $\omega_{2}(D)= \pm \omega(K)^{2}$, ie we define $\omega_{2}(D)^{-1}$ to be a modification of the Hessian of the potential function obtained from $D$ (Definition 4.4). Further, from a parameterized knot diagram, we construct a monodromy representation of a knot group into $\mathrm{PGL}_{2} \mathbb{C}$ (Section 3.1), and we can consider the twisted Reidemeister torsion associated with such a monodromy representation. The following theorem is the main theorem of this paper, which confirm the above mentioned conjecture of $\omega(K)$ for any two-bridge knot assuming the above mentioned technical assumptions of the Poisson summation formula and the saddle point method.

Theorem 1.1 Let $K$ be any two-bridge knot, and let $D$ be an appropriate parameterized diagram of $K$. Then

$$
\omega_{2}(D)= \pm \frac{\tau(K)}{2 \sqrt{-1}}
$$

where $\tau(K)$ is the twisted Reidemeister torsion associated with the monodromy representation obtained from the parameterization of $D$.

We remark (see Remark D.14) that for any hyperbolic two-bridge knot, ${ }^{1}$ the holonomy representation of the complete hyperbolic structure of the knot complement can always be constructed from a parameterized knot diagram, and we can apply the theorem to such a case.

${ }^{1}$ In general, for many hyperbolic two-bridge knots, all parabolic representations can be constructed from parameterized knot diagrams, but there are some exceptional cases; see Remark D.12 for details. For the case of nonhyperbolic two-bridge knots, we can also apply Theorem 1.1 to many parabolic representations; see Appendix E for details. 
We apply the theorem in Examples 3.4, 3.5, 4.10 and 4.11 for the $\overline{5_{2}}$ knot and the $\overline{6_{1}}$ knot with the holonomy representations of the hyperbolic structures, and obtain that the values of $\omega(K)$ and $\tau(K)$ are numerically given by

$$
\begin{aligned}
& \omega\left(\overline{5_{2}}\right)=0.09019057740 \ldots+\sqrt{-1} \cdot 0.6499757866 \ldots, \\
& \tau\left(\overline{5_{2}}\right)=-0.2344867659 \ldots-\sqrt{-1} \cdot 0.8286683659 \ldots, \\
& \omega\left(\overline{6_{1}}\right)=-0.5213883634 \ldots+\sqrt{-1} \cdot 0.07173228265 \ldots, \\
& \tau\left(\overline{6_{1}}\right)=0.1496015098 \ldots+\sqrt{-1} \cdot 0.5334006103 \ldots,
\end{aligned}
$$

where we can confirm that the values of $\omega\left(\overline{5_{2}}\right)$ and $\omega\left(\overline{6_{1}}\right)$ are equal to the values given in $[17 ; 20]$, and the values of $\tau\left(\overline{5_{2}}\right)$ and $\tau\left(\overline{6_{1}}\right)$ are equal to the values obtained from Tran [26] (see Examples 3.4 and 3.5). Hence, we can numerically verify the theorem by

$$
\begin{aligned}
& \omega\left(\overline{5_{2}}\right)^{2}=-0.4143341829 \ldots+\sqrt{-1} \cdot 0.1172433829 \ldots=\frac{\tau\left(\overline{5_{2}}\right)}{2 \sqrt{-1}}, \\
& \omega\left(\overline{6_{1}}\right)^{2}=0.2667003051 \ldots-\sqrt{-1} \cdot 0.07480075491 \ldots=\frac{\tau\left(\overline{6_{1}}\right)}{2 \sqrt{-1}} .
\end{aligned}
$$

Further, by results in $[17 ; 18 ; 20]$, the theorem means that the above mentioned conjecture on $\omega(K)$ is confirmed, as

$$
\omega(K)^{2}= \pm \frac{\tau(K)}{2 \sqrt{-1}}
$$

for any hyperbolic knot with up to 7 crossings, since they are two-bridge knots.

The theorem means that the Hessian of the potential function is related to the twisted Reidemeister torsion. We explain how they are related, roughly speaking, as follows. As mentioned above, the twisted Reidemeister torsion of the problem is the Reidemeister torsion of the cochain complex of the knot complement with the $\mathfrak{s l}_{2} \mathbb{C}$ coefficient twisted by the adjoint action of the holonomy representation of the hyperbolic structure of the knot complement. This Reidemeister torsion is determined by the alternating product of the determinants of the coboundary maps of this cochain complex; in particular, its essential factor is the determinant of the coboundary map $d_{1}: C^{1} \rightarrow C^{2}$ with respect to an appropriate basis. Further, it is well known that $H^{1}$ of this cochain complex is naturally isomorphic to the tangent space of the space of conjugacy classes of $\mathrm{PGL}_{2} \mathbb{C}$ representations of the knot group. Hence, roughly speaking, the twisted Reidemeister torsion is given by the determinant of the matrix whose entries are the coefficients of the defining equations of the tangent space of the representation space. On the other hand, we can reconstruct the representation space by using an ideal tetrahedral decomposition 
of the knot complement. The shape of an ideal tetrahedron is parameterized by the crossratio of the coordinates of its four vertices, and the representation space is parameterized by solutions of hyperbolicity equations of such parameters. Further, the hyperbolicity equations are given by differentials of the potential function. Hence, the tangent space of the representation space is presented by the Hesse matrix of the potential function, and its determinant (ie the Hessian of the potential function) is expected to be related to the twisted Reidemeister torsion, as mentioned above.

We explain an outline of the proof of the theorem. We consider a parameterized knot diagram of an open two-bridge knot, where an open knot is a 1-tangle whose closure is a knot. We decompose such a knot diagram into elementary tangle diagrams. Further, we reformulate $\tau(K)$ and $\omega_{2}(D)$ as compositions of operator invariants of such elementary diagrams. In other words, regarding an open two-bridge knot as a plat closure of a 3-braid, we reformulate $\tau(K)$ and $\omega_{2}(D)$ in terms of "representations" of parameterized 3-braids. Finally, we prove the theorem by comparing recursive formulas of both sides of the required formula of the theorem.

The paper is organized as follows. In Section 2, we review some basic facts used in this paper, such as the definition of the Kashaev invariant and a parameterization of a knot diagram by hyperbolicity parameters. In Section 3, we explain how we calculate the twisted Reidemeister torsion for two-bridge knots. We construct a monodromy representation of a knot group into $\mathrm{PGL}_{2} \mathbb{C}$ from a parameterized knot diagram, and calculate the twisted Reidemeister torsion associated with this monodromy representation by decomposing a two-bridge knot diagram into elementary tangle diagrams. In Section 4 , we define $\omega_{2}(D)$ for an oriented parameterized open knot diagram $D$, and show a relation of it to the Kashaev invariant, and calculate it for two-bridge knots. In Section 5, we show a proof of Theorem 1.1, by comparing recursive formulas of both sides of the required formula of the theorem. In the appendices, we explain some supplementary topics.

The authors would like to thank Stavros Garoufalidis, Sergei Gukov, Kazuo Habiro, Rinat Kashaev and Hitoshi Murakami for helpful comments. The authors would also like to thank the referees for helpful suggestions and careful reading of the manuscript.

\section{Preliminaries}

In this section, we review some basic facts used in this paper. In Section 2.1, we review the definition of the Kashaev invariant. In Section 2.2, we review a parameterization of a knot diagram by hyperbolicity parameters. 


\subsection{Kashaev invariant}

In this section, we review the definition of the Kashaev invariant following Yokota [32], and review some related formulas.

Let $N$ be an integer $\geq 2$. We set $q=\exp (2 \pi \sqrt{-1} / N)$, and let

$$
(x)_{n}=(1-x)\left(1-x^{2}\right) \cdots\left(1-x^{n}\right)
$$

for $n \geq 0$. It is known [15] that for any $n, m$ with $n \leq m$,

$$
\begin{aligned}
(q)_{n}(\bar{q})_{N-n-1} & =N \\
\sum_{n \leq k \leq m} \frac{1}{(q)_{m-k}(\bar{q})_{k-n}} & =1
\end{aligned}
$$

Following Faddeev [7], we define a holomorphic function $\varphi(t)$ on $\{t \in \mathbb{C} \mid 0<\operatorname{Re} t<1\}$ by

$$
\varphi(t)=\int_{-\infty}^{\infty} \frac{e^{(2 t-1) x} d x}{4 x \sinh x \sinh (x / N)}
$$

noting that this integrand has poles at $n \pi \sqrt{-1}(n \in \mathbb{Z})$, where, to avoid the pole at 0 , we choose the following contour for the integral:

$$
(-\infty,-1] \cup\{z \in \mathbb{C}|| z \mid=1, \operatorname{Im} z \geq 0\} \cup[1, \infty) .
$$

It is known (see Faddeev, Kashaev and Volkov [8] and Woronowicz [30]) that

$$
\begin{aligned}
& (q)_{n}=\exp \left(\varphi\left(\frac{1}{2 N}\right)-\varphi\left(\frac{2 n+1}{2 N}\right)\right) \\
& (\bar{q})_{n}=\exp \left(\varphi\left(1-\frac{2 n+1}{2 N}\right)-\varphi\left(1-\frac{1}{2 N}\right)\right) .
\end{aligned}
$$

Further, it is also known [8; 30] (see also [17]) that

$$
\begin{aligned}
& \frac{1}{N} \varphi(t)=\frac{1}{2 \pi \sqrt{-1}} \operatorname{Li}_{2}\left(e^{2 \pi \sqrt{-1} t}\right)+O\left(\frac{1}{N^{2}}\right), \\
& \frac{1}{N} \varphi^{\prime}(t)=-\log \left(1-e^{2 \pi \sqrt{-1} t}\right)+O\left(\frac{1}{N^{2}}\right) .
\end{aligned}
$$

Furthermore, it is known (due to Kashaev; see [17]) that

$$
\begin{aligned}
\varphi\left(\frac{1}{2 N}\right) & =\frac{N}{2 \pi \sqrt{-1}} \frac{\pi^{2}}{6}+\frac{1}{2} \log N+\frac{\pi \sqrt{-1}}{4}-\frac{\pi \sqrt{-1}}{12 N}, \\
\varphi\left(1-\frac{1}{2 N}\right) & =\frac{N}{2 \pi \sqrt{-1}} \frac{\pi^{2}}{6}-\frac{1}{2} \log N+\frac{\pi \sqrt{-1}}{4}-\frac{\pi \sqrt{-1}}{12 N} .
\end{aligned}
$$


Following Yokota $[32]^{2}$, we review the definition of the Kashaev invariant. We set

$$
\mathcal{N}=\{0,1, \ldots, N-1\} .
$$

For $i, j, k, l \in \mathcal{N}$, we set

$$
\begin{aligned}
R_{k l}^{i j} & =\frac{N q^{-1 / 2+i-k} \theta_{k l}^{i j}}{(q)_{[i-j]}(\bar{q})_{[j-l]}(q)_{[l-k-1]}(\bar{q})_{[k-i]}}, \\
\bar{R}_{k l}^{i j} & =\frac{N q^{1 / 2+j-l} \theta_{k l}^{i j}}{(\bar{q})_{[i-j]}(q)_{[j-l]}(\bar{q})_{[l-k-1]}(q)_{[k-i]}},
\end{aligned}
$$

where $[m] \in \mathcal{N}$ denotes the residue of $m$ modulo $N$, and we set

$$
\theta_{k l}^{i j}= \begin{cases}1 & \text { if }[i-j]+[j-l]+[l-k-1]+[k-i]=N-1, \\ 0 & \text { otherwise }\end{cases}
$$

Let $K$ be an oriented knot. We consider a 1 -tangle whose closure is isotopic to $K$ such that its string is oriented downward at its endpoints; abusing the notation, we also denote this 1 -tangle by $K$, and call such a 1-tangle an open knot. Let $D$ be a diagram of this 1 -tangle. We present $D$ by a union of elementary tangle diagrams shown in (8). We decompose the string of $D$ into edges by cutting it at crossings and critical points with respect to the height function of $\mathbb{R}^{2}$. A labeling is an assignment of an element of $\mathcal{N}$ to each edge. Here, we assign 0 to the two edges adjacent to the endpoints of $D$. We define the weights of labeled elementary tangle diagrams by

$$
W\left(\searrow_{k}^{i} \searrow_{l}^{j}\right)=R_{k l}^{i j}, \quad W\left(\searrow_{k}^{i}\right)=R_{k l}^{i j},
$$$$
W\left(\bigcap_{k}\right)=q^{-1 / 2} \delta_{k, l-1}, \quad W\left(\bigcap_{l}\right)=\delta_{k, l},
$$$$
W\left((\lambda)=q^{1 / 2} \delta_{i, j+1}, W\left(\jmath^{i}\right)=\delta_{i, j} .\right.
$$

${ }^{2}$ We make a minor modification of the definition of weights of critical points from the definition in [32], in order to make $\langle K\rangle_{N}$ invariant under Reidemeister moves. 
Then the Kashaev invariant $\langle K\rangle_{N}$ of $K$ is defined by

$$
\langle K\rangle_{N}=\sum_{\text {labelings crossings }} \prod_{\text {of } D} W(\text { crossings }) \prod_{\substack{\text { critical } \\ \text { points of } D}} W(\text { critical points }) \in \mathbb{C} .
$$

\subsection{Knot diagrams parameterized by hyperbolicity parameters}

In this section, we review a parameterization of an open knot diagram by hyperbolicity parameters, following Yokota [31]. Further, we review a potential function of a parameterized open knot diagram.

We parameterize edges of an open knot diagram by parameters in $\mathbb{C} \cup\{\infty\}$, for example, as follows.

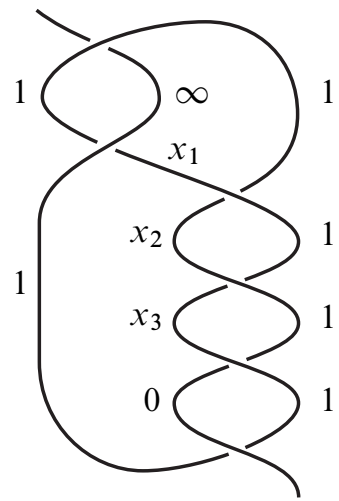

We parameterize edges adjacent to unbounded regions by 1 . We parameterize edges next to the terminal edges by 0 or $\infty$ as shown above; we parameterize such an edge by $\infty$ (resp. 0 ) if it is connected to the terminal edge by an underpath (resp. an overpath). We parameterize the other edges in such a way that the parameters belong to $\mathbb{C}-\{0\}$, and satisfy the hyperbolicity equations, which are given by

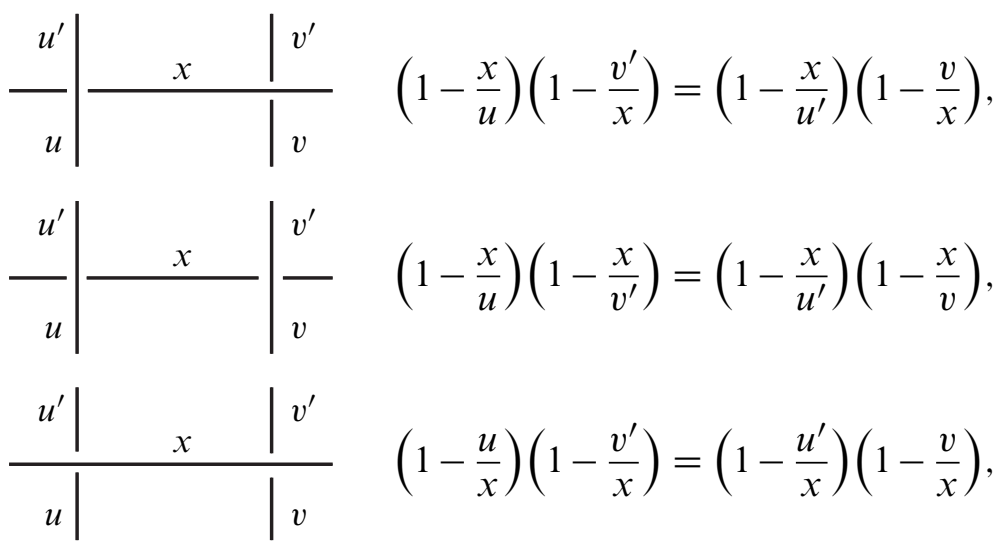


where we assume that the values of both sides of the equations are nonzero. We call such parameters hyperbolicity parameters. For example, for the knot diagram (9), the hyperbolicity equations are given by

$$
\begin{aligned}
1-\frac{x_{2}}{x_{1}} & =\left(1-x_{1}\right)\left(1-\frac{1}{x_{1}}\right), \\
\left(1-\frac{x_{2}}{x_{1}}\right)\left(1-\frac{1}{x_{2}}\right) & =\left(1-x_{2}\right)\left(1-\frac{x_{3}}{x_{2}}\right), \\
\left(1-\frac{x_{3}}{x_{2}}\right)\left(1-\frac{1}{x_{3}}\right) & =1-x_{3} .
\end{aligned}
$$

We can verify by concrete calculation that each solution of the above equations is isolated (ie 0-dimensional). ${ }^{3}$ Further, as we explain in Section 3.1, such a parameterization gives a monodromy representation of the knot group into $\mathrm{PGL}_{2} \mathbb{C}$.

We consider an open knot diagram parameterized by hyperbolicity parameters. We consider an angle consisting of two adjacent edges at a crossing. We associate such an angle with the value
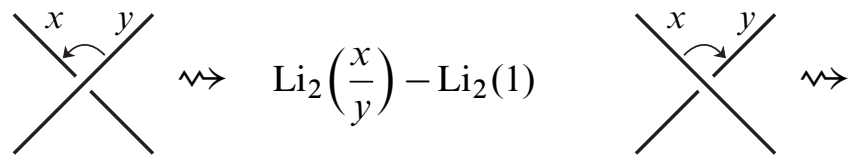

$$
\mathrm{Li}_{2}(1)-\mathrm{Li}_{2}\left(\frac{y}{x}\right)
$$

where we consider the orientation of an angle from the overpath to the underpath, the left case is when this orientation is counterclockwise, and the right case is when this orientation is clockwise. We recall that $\operatorname{Li}_{2}(1)=\pi^{2} / 6$. For a parameterized open knot diagram, we define the potential function $V$ to be the sum of such values for all angles except for the constant terms, regarding $V$ as a function of hyperbolicity parameters:

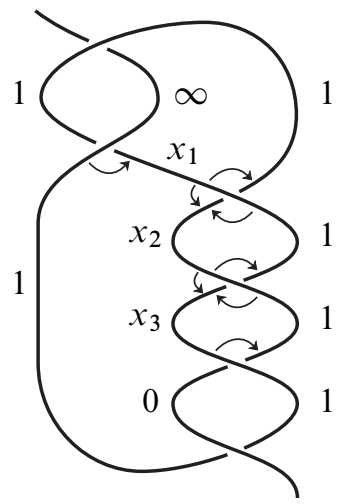

${ }^{3}$ For any two-bridge knot, the system of hyperbolicity equations can be rewritten as a single polynomial equation of $x_{1}$ as we explain in Section 5. Hence each solution of it is isolated, for any two-bridge knot. On the other hand, for a general knot, the space of solutions of hyperbolicity equations might not necessarily be 0 -dimensional; see Remark 4.3. 
For example, for the above knot diagram, the potential function $V$ is given by

$$
\begin{aligned}
V\left(x_{1}, x_{2}, x_{3}\right)=\mathrm{Li}_{2}( & \left.x_{1}\right)-\mathrm{Li}_{2}\left(\frac{1}{x_{1}}\right)+\mathrm{Li}_{2}\left(\frac{x_{2}}{x_{1}}\right)-\mathrm{Li}_{2}\left(x_{2}\right) \\
& -\mathrm{Li}_{2}\left(\frac{1}{x_{2}}\right)+\mathrm{Li}_{2}\left(\frac{x_{3}}{x_{2}}\right)-\mathrm{Li}_{2}\left(x_{3}\right)-\mathrm{Li}_{2}\left(\frac{1}{x_{3}}\right)+2 \mathrm{Li}_{2}(1) .
\end{aligned}
$$

We note that

$$
x \frac{\partial}{\partial x} \operatorname{Li}_{2}\left(\frac{x}{y}\right)=-\log \left(1-\frac{x}{y}\right), \quad y \frac{\partial}{\partial y} \operatorname{Li}_{2}\left(\frac{x}{y}\right)=\log \left(1-\frac{x}{y}\right) .
$$

We also note that the hyperbolicity equations are given by

$$
\frac{\partial}{\partial x_{i}} V=0 \text { for all } i
$$

and, hence, a solution of the hyperbolicity equations gives a critical point of $V$.

\section{Calculation of the twisted Reidemeister torsion}

In this section, we explain how we calculate the twisted Reidemeister torsion for twobridge knots. In Section 3.1, we explain how we calculate the monodromy representation of a knot group into $\mathrm{PGL}_{2} \mathbb{C}$ when a knot diagram is parameterized by hyperbolicity parameters. In Section 3.2, we explain how we calculate the twisted Reidemeister torsion for the $\overline{5_{2}} \mathrm{knot}$, as the simplest example among two-bridge knots; the calculation is reduced to the calculations of

$$
\operatorname{det}\left(\hat{E}_{2} \hat{D}_{1} \widehat{E}_{1}\right) \text { and } \operatorname{det}\left(\check{D}_{1} \check{E}_{1}\right) \text {, }
$$

where we introduce this notation in Section 3.2. In Section 3.3, we decompose open twobridge knot diagrams into elementary tangle diagrams, to formulate such calculations for any two-bridge knot. In Sections 3.4 and 3.5, we calculate

$$
\operatorname{det}\left(\widehat{E}_{2} \widehat{D}_{1} \widehat{E}_{1}\right) \text { and } \operatorname{det}\left(\check{D}_{1} \check{E}_{1}\right)
$$

respectively for any two-bridge knot. By using the results, we calculate the twisted Reidemeister torsion for any two-bridge knot in Section 3.6. See also Dubois, Huynh and Yamaguchi [6] and [26] for the calculation of the Reidemeister torsion for twist knots. 


\subsection{The monodromy representation}

In this section, we explain how we calculate the monodromy representation of a knot group into $\mathrm{PGL}_{2} \mathbb{C}$ from a parameterized knot diagram.

We review how to make an ideal tetrahedral decomposition of $S^{3}-K$ from a knot diagram, following Thurston $[25 ; 31]$. There are four tetrahedra at each crossing of the knot diagram, and, by making an octahedron as the union of such four tetrahedra at each crossing, we obtain an octahedral decomposition of $S^{3}-K$. As in [31], we associate a complex parameter to each edge of the knot diagram, and consider the hyperbolicity equations with respect to the parameters. Then the shape of an ideal octahedron at each crossing is determined as follows:
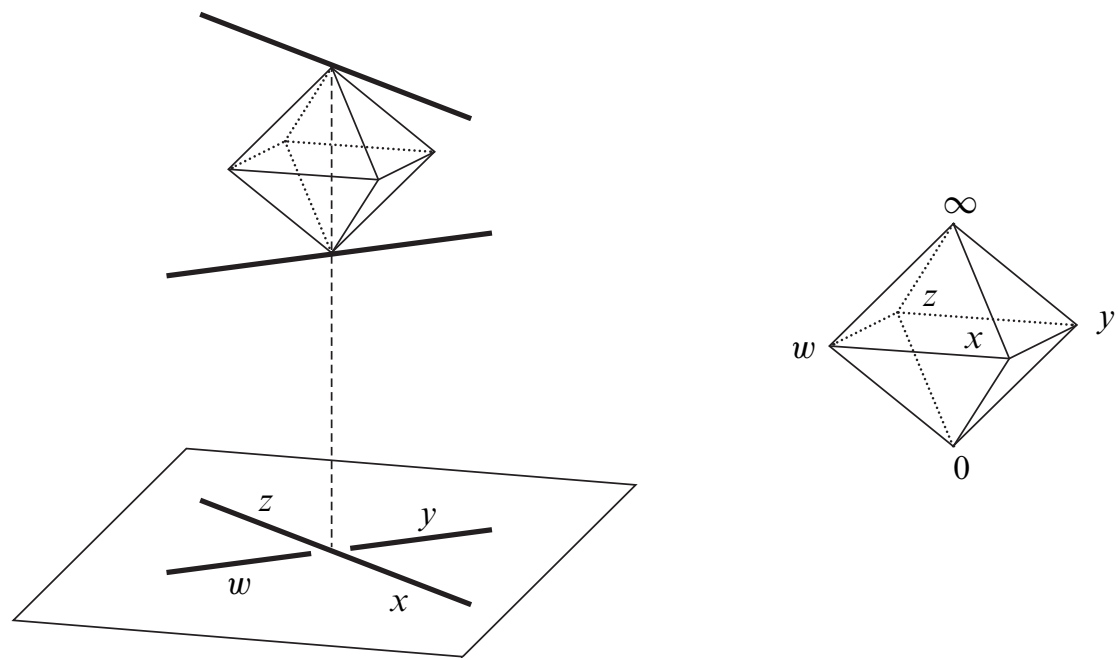

We can glue ideal tetrahedra at each face of a knot diagram. For example, we can make the polyhedron of the following right picture by gluing 5 tetrahedra at the face of the left picture:
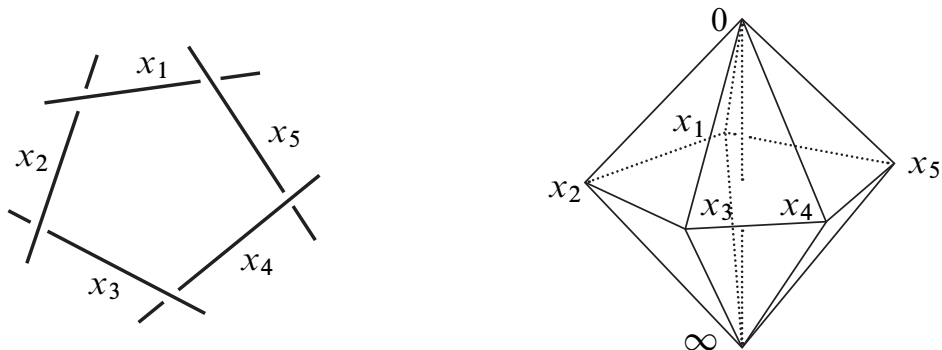
Here, we note that the edge $\overline{x_{1} x_{2}}$ of the tetrahedron " $\infty 0 x_{1} x_{2}$ " at the crossing of the edges of $x_{1}$ and $x_{2}$ in the left picture corresponds to the edge $\overline{\infty 0}$ of the tetrahedron " $0 \infty x_{1} x_{2}$ " of the right picture.

We consider the following left picture as a part of a knot diagram:
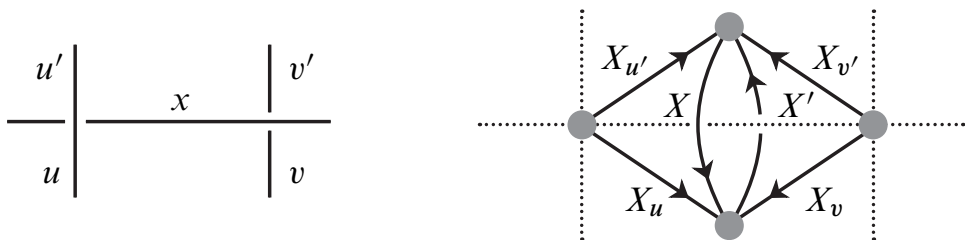

In the right picture, we consider tetrahedra at each crossing as in (12), and move them to tetrahedra at each face as in (13) by the maps $X_{u}, X_{u^{\prime}}, X_{v}, X_{v^{\prime}} \in \mathrm{PGL}_{2} \mathbb{C}$, and calculate $X$ and $X^{\prime}$ by using these maps ${ }^{4}$ as follows. As mentioned in Section 2.2, the hyperbolicity equation of these parameters is

$$
\left(1-\frac{x}{u}\right)\left(1-\frac{v^{\prime}}{x}\right)=\left(1-\frac{x}{u^{\prime}}\right)\left(1-\frac{v}{x}\right)
$$

We consider tetrahedra at each crossing as in (12), and consider tetrahedra at each face as in (13). Further, we consider maps taking such tetrahedra to each other as in the right picture; for example, the map $X_{u}$ in the right picture takes a tetrahedron at the left crossing placed as in (12) to a tetrahedron at the lower face placed as in (13). Such maps take vertices of the tetrahedra as follows:
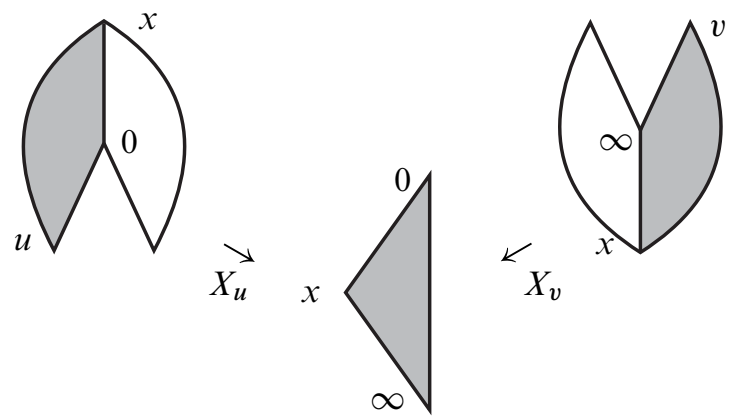

Hence

$$
\begin{aligned}
& X_{u}(x)=0, \quad X_{u}(0)=x, \quad X_{u}(u)=\infty, \\
& X_{v}(v)=0, \quad X_{v}(\infty)=x, \quad X_{v}(x)=\infty,
\end{aligned}
$$

${ }^{4}$ This means that $X_{u}, X_{u^{\prime}}, X_{v}$ and $X_{v^{\prime}}$ transform coordinates of (12) to coordinates of (13), and $X$ and $X^{\prime-1}$ transform the coordinates of (13) in the upper face to the coordinates of (13) in the lower face. 
where $\mathrm{PGL}_{2} \mathbb{C}$ acts on $\mathbb{C} \cup\{\infty\}$ by the Möbius transformation. It follows that

$$
\begin{aligned}
X_{u} & \sim\left(\begin{array}{cc}
1 & -x \\
1 / u & -1
\end{array}\right), \\
X_{v} & \sim\left(\begin{array}{cc}
1 & -v \\
1 / x & -1
\end{array}\right),
\end{aligned}
$$

where " " means the equality in $\mathrm{PGL}_{2} \mathbb{C}$. Similarly, we have that

$$
\begin{aligned}
X_{u^{\prime}} & \sim\left(\begin{array}{cc}
1 & -x \\
1 / u^{\prime} & -1
\end{array}\right), \\
X_{v^{\prime}} & \sim\left(\begin{array}{cc}
1 & -v^{\prime} \\
1 / x & -1
\end{array}\right) .
\end{aligned}
$$

Therefore,

$$
\begin{gathered}
X=X_{u} X_{u^{\prime}}^{-1} \sim\left(\begin{array}{cc}
\frac{x}{u^{\prime}}-1 & 0 \\
\frac{1}{u^{\prime}}-\frac{1}{u} & \frac{x}{u}-1
\end{array}\right), \\
X^{\prime}=X_{v^{\prime}} X_{v}^{-1} \sim\left(\begin{array}{cc}
\frac{v^{\prime}}{x}-1 & v-v^{\prime} \\
0 & \frac{v}{x}-1
\end{array}\right) .
\end{gathered}
$$

We note that, from the construction, $X$ fixes 0 and $x$, and $X^{\prime}$ fixes $\infty$ and $x$ by the Möbius transformation.

By using such matrices, we can calculate the monodromy representation $\pi_{1}\left(S^{3}-K\right) \rightarrow$ $\mathrm{PGL}_{2} \mathbb{C}$ from a knot diagram with parameters.

\subsection{Calculation of the twisted Reidemeister torsion for the $\overline{5_{2}}$ knot}

In this section, we explain how we calculate the twisted Reidemeister torsion for the $\overline{5_{2}}$ knot, before we explain the calculation for any two-bridge knot later. We calculate it in the following 6 steps. In Step 1, we calculate the monodromy representation of the knot group of the $\overline{5_{2}}$ knot. In Step 2, we give the cochain complex $C^{*}$ of the knot complement with the $\mathfrak{s l}_{2} \mathbb{C}$ coefficient twisted by the monodromy representation of Step 1. In Step 3, we give a subcomplex $\widehat{C}^{*}$ of $C^{*}$, and we set $\check{C}^{*}=C^{*} / \widehat{C}^{*}$. In Steps 4 and 5, we calculate the Reidemeister torsions of $\widehat{C}^{*}$ and $\check{C}^{*}$ respectively. In Step 6, we present the Reidemeister torsion of the knot as the product of the Reidemeister torsions of $\widehat{C}^{*}$ and $\check{C}^{*}$.

Step 1 In this step, we calculate the monodromy representation of the knot group of the $\overline{5_{2}}$ knot. 
The $\overline{5_{2}}$ knot is the knot presented by the following picture; it is the mirror image of the $5_{2}$ knot:
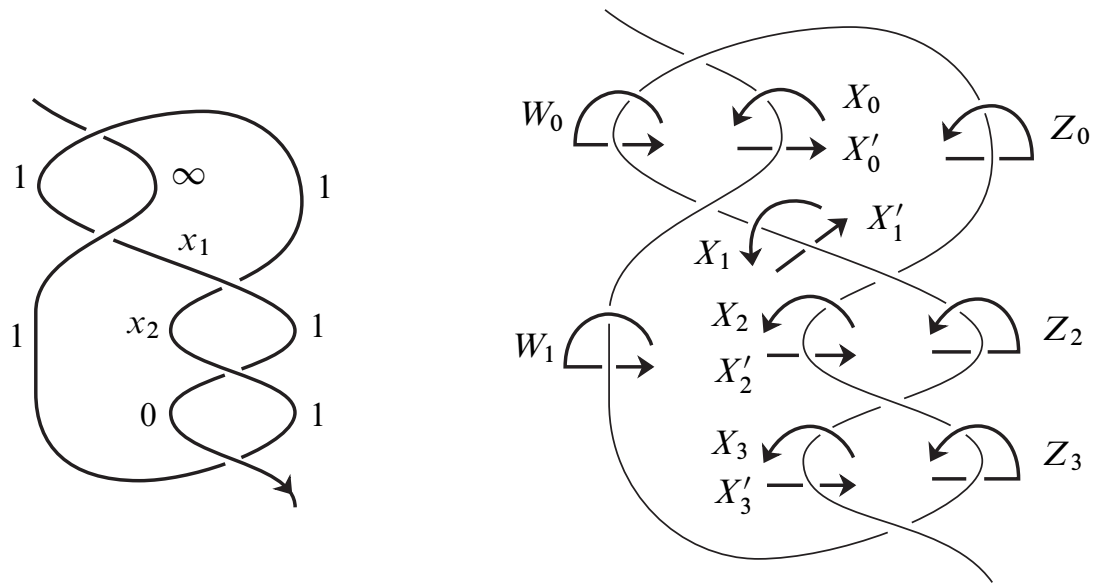

We calculate $X_{i}, X_{i}^{\prime}, W_{i}, Z_{i} \in \mathrm{PGL}_{2} \mathbb{C}$ as follows; we note that we do not use the notation $W_{2}, W_{3}$ and $Z_{1}$ here, while we also use them in the general case later. As in [31], the parameters of the knot diagram is given as in the left picture. The hyperbolicity equations are

$$
\left(1-x_{1}\right)\left(1-\frac{1}{x_{1}}\right)=1-\frac{x_{2}}{x_{1}}, \quad\left(1-\frac{x_{2}}{x_{1}}\right)\left(1-\frac{1}{x_{2}}\right)=1-x_{2} .
$$

Hence,

$$
x_{2}=x_{1}^{2}-x_{1}+1, \quad x_{2}+1-\frac{x_{2}}{x_{1}}=0 .
$$

We calculate $X_{i}$ and $X_{i}^{\prime}$ by the way of Section 3.1; for example,

$$
\begin{aligned}
& X_{0}^{\prime} \sim\left(\begin{array}{cc}
1 & x_{1}-1 \\
0 & 1
\end{array}\right), \quad X_{1} \sim\left(\begin{array}{cc}
1 & 0 \\
1 & 1-x_{1}
\end{array}\right), \quad X_{1}^{\prime} \sim\left(\begin{array}{cc}
\frac{1}{x_{1}}-1 & x_{2}-1 \\
0 & \frac{x_{2}}{x_{1}}-1
\end{array}\right), \\
& X_{2} \sim\left(\begin{array}{cc}
x_{2}-1 & 0 \\
1-\frac{1}{x_{1}} & \frac{x_{2}}{x_{1}}-1
\end{array}\right), \quad X_{2}^{\prime} \sim\left(\begin{array}{cc}
1-\frac{1}{x_{2}} & 1 \\
0 & 1
\end{array}\right), \quad X_{3} \sim\left(\begin{array}{cc}
1 & 0 \\
\frac{1}{x_{2}}-1 & 1
\end{array}\right) .
\end{aligned}
$$

By using them, we can calculate the other matrices; for example,

$$
\begin{array}{ll}
Z_{2} \sim X_{2}^{-1} X_{1} X_{1}^{\prime} X_{2} \sim\left(\begin{array}{cc}
0 & 1 \\
-1 & 2
\end{array}\right), & X_{3}^{\prime} \sim X_{3}^{-1} X_{2}^{\prime-1} Z_{2} X_{2}^{\prime} \sim\left(\begin{array}{ll}
1 & 0 \\
0 & 1
\end{array}\right), \\
W_{1} \sim X_{3}^{\prime-1} Z_{3}^{-1} X_{3}^{\prime} \sim\left(\begin{array}{cc}
2 & -1 \\
1 & 0
\end{array}\right), & X_{0} \sim X_{0}^{\prime-1} X_{1}^{-1} W_{1} X_{1} \sim\left(\begin{array}{ll}
1 & 0 \\
0 & 1
\end{array}\right) .
\end{array}
$$


In similar ways, we can show that

$$
W_{i} \sim\left(\begin{array}{cc}
2 & -1 \\
1 & 0
\end{array}\right), \quad Z_{i} \sim\left(\begin{array}{cc}
0 & 1 \\
-1 & 2
\end{array}\right)
$$

for each $i$.

Step 2 In this step, we give the cochain complex $C^{*}$ of the knot complement with the $\mathfrak{s l}_{2} \mathbb{C}$ coefficient twisted by the monodromy representation of Step 1.

We consider a cellular decomposition of the knot complement as follows. The (large) 0 -cell is a shaded region of the following left picture. The 1-cells are the arrows of the following left picture. The 2-cells are given as in the right two pictures:
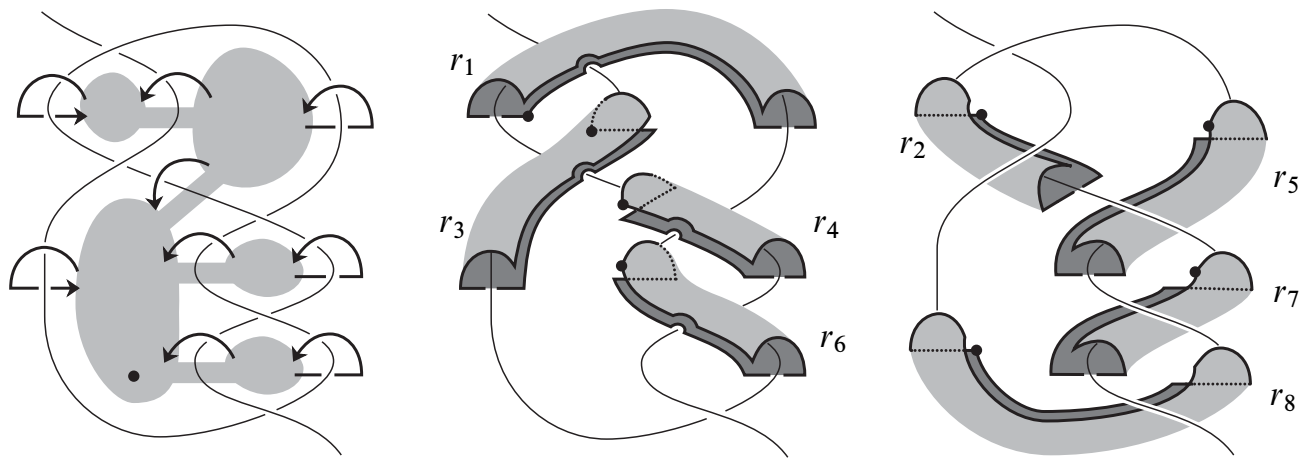

Here the base points of the 0 -cell and the 2-cells are depicted by dots in the pictures, and the base points of the 1-cells are the tops of the arrows. This cellular decomposition is a modification of the dual of the ideal tetrahedral decomposition mentioned in Section 3.1; see Appendix A for details.

We consider the cochain complex $C^{*}$ of this cellular decomposition with the $\mathfrak{s l}_{2} \mathbb{C}$ coefficient twisted by the monodromy representation of Step 1 . The relator given by the $2-$ cell $r_{1}$ is presented by

$$
W_{0} X_{0} Z_{0} X_{0}^{-1}
$$

Its perturbation is given by

$$
\left(1+\varepsilon e_{W_{0}}\right) W_{0} \cdot\left(1+\varepsilon e_{X_{0}}\right) X_{0} \cdot\left(1+\varepsilon e_{Z_{0}}\right) Z_{0} \cdot X_{0}^{-1}\left(1-\varepsilon e_{X_{0}}\right)+O\left(\varepsilon^{2}\right)
$$

for $e_{W_{0}}, e_{X_{0}}, e_{Z_{0}} \in \mathfrak{s l}_{2} \mathbb{C}$. Its coefficient of $\varepsilon$ is presented by

$$
e_{r_{1}}=e_{W_{0}}+\left(\mathcal{W}_{0}-1\right) e_{X_{0}}+\mathcal{W}_{0} e_{Z_{0}}
$$


where we set $\mathcal{W}_{i}=\operatorname{ad}\left(W_{i}\right), \mathcal{X}_{i}=\operatorname{ad}\left(X_{i}\right), \mathcal{Z}_{i}=\operatorname{ad}\left(Z_{i}\right), \ldots$ Similarly, from the relator $W_{0} X_{0}^{\prime-1} X_{1}^{-1} X_{1}^{\prime-1} X_{0}^{\prime}$ of the $2-$ cell $r_{2}$, we obtain

$$
e_{r_{2}}=e_{W_{0}}-\mathcal{X}_{0}^{\prime-1} \mathcal{X}_{1}^{\prime} e_{X_{1}}
$$

Further, from the relator $X_{0} X_{1}^{-1} W_{1}^{-1} X_{1} X_{0}^{\prime}$ of the $2-$ cell $r_{3}$, we obtain

$$
e_{r_{3}}=e_{X_{0}}-\mathcal{X}_{0}^{\prime-1} \mathcal{X}_{1}^{-1} e_{W_{1}}+\mathcal{X}_{1}^{-1}\left(\mathcal{W}_{1}^{-1}-1\right) e_{X_{1}} .
$$

By calculating similarly, the coboundary map $D_{1}: C^{1} \rightarrow C^{2}$ is presented by

$$
D_{1}=\left(\begin{array}{ccccccccc}
1 & \mathcal{W}_{0}-1 & \mathcal{W}_{0} & 0 & 0 & 0 & 0 & 0 & 0 \\
1 & 0 & 0 & 0 & -\mathcal{X}_{0}^{\prime-1} \mathcal{X}_{1}^{\prime} & 0 & 0 & 0 & 0 \\
0 & 1 & 0 & -\mathcal{X}_{0}^{\prime-1} \mathcal{X}_{1}^{-1} & \mathcal{X}_{1}^{-1}\left(\mathcal{W}_{1}^{-1}-1\right) & 0 & 0 & 0 & 0 \\
0 & 0 & 0 & 0 & 1 & \mathcal{X}_{1} \mathcal{X}_{1}^{\prime}-1 & -\mathcal{X}_{2} & 0 & 0 \\
0 & 0 & 1 & 0 & 0 & -\mathcal{X}_{1}^{\prime} & 0 & 0 & 0 \\
0 & 0 & 0 & 0 & 0 & 1 & 0 & \mathcal{X}_{2} \mathcal{X}_{2}^{\prime}-1 & -\mathcal{X}_{3} \\
0 & 0 & 0 & 0 & 0 & 0 & 1 & -\mathcal{X}_{2}^{\prime} & 0 \\
0 & 0 & 0 & 1 & 0 & 0 & 0 & 0 & \mathcal{Z}_{3}^{-1}
\end{array}\right),
$$

with respect to the basis $\left(e_{W_{0}}, e_{X_{0}}, e_{Z_{0}}, e_{W_{1}}, e_{X_{1}}, e_{X_{2}}, e_{Z_{2}}, e_{X_{3}}, e_{Z_{3}}\right)$ of $C^{1}$ and the basis $\left(e_{r_{1}}, e_{r_{2}}, e_{r_{3}}, \ldots, e_{r_{8}}\right)$ of $C^{2}$. Further, the coboundary map $D_{0}: C^{0} \rightarrow C^{1}$ is presented by a matrix of the form

$$
D_{0}=\left(\begin{array}{c}
\left(\mathcal{W}_{0}-1\right) \mathcal{X}_{0}^{\prime-1} \mathcal{X}_{1}^{\prime} \\
\left(\mathcal{X}_{0}-\mathcal{X}_{0}^{\prime-1}\right) \mathcal{X}_{1}^{\prime} \\
\left(\mathcal{Z}_{0}-1\right) \mathcal{X}_{1}^{\prime} \\
\mathcal{W}_{1}-1 \\
\mathcal{X}_{1} \mathcal{X}_{1}^{\prime}-1 \\
\mathcal{X}_{2} \mathcal{X}_{2}^{\prime}-1 \\
\left(\mathcal{Z}_{2}-1\right) \mathcal{X}_{2}^{\prime} \\
\mathcal{X}_{3} \mathcal{X}_{3}^{\prime}-1 \\
\left(\mathcal{Z}_{3}-1\right) \mathcal{X}_{3}^{\prime}
\end{array}\right)=\left(\begin{array}{c} 
\\
\vdots \\
\vdots \\
\mathcal{X}_{3}-1 \\
\mathcal{Z}_{3}-1
\end{array}\right)
$$

with respect to the basis $\left(e_{W_{0}}, e_{X_{0}}, e_{Z_{0}}, e_{W_{1}}, e_{X_{1}}, e_{X_{2}}, e_{Z_{2}}, e_{X_{3}}, e_{Z_{3}}\right)$ of $C^{1}$.

Step 3 In this step, we give a subcomplex $\widehat{C}^{*}$ of $C^{*}$.

Since $X_{i}$ and $X_{i}^{\prime}$ have fixed points (Section 3.1), modify $D_{1}$ by multiplying by

$$
\begin{array}{r}
\operatorname{diag}\left(\operatorname{ad}\left(\begin{array}{ll}
1 & 1 \\
1 & 0
\end{array}\right)^{-1} \operatorname{ad}\left(\begin{array}{ll}
1 & 1 \\
1 & 0
\end{array}\right)^{-1} 1 \operatorname{ad}\left(\begin{array}{cc}
x_{1} & 1 \\
1 & 0
\end{array}\right)^{-1} \operatorname{ad}\left(\begin{array}{ll}
1 & 1 \\
1 & 0
\end{array}\right)^{-1}\right. \\
\left.\operatorname{ad}\left(\begin{array}{cc}
x_{2} & 1 \\
1 & 0
\end{array}\right)^{-1} \operatorname{ad}\left(\begin{array}{ll}
1 & 1 \\
1 & 0
\end{array}\right)^{-1} \operatorname{ad}\left(\begin{array}{ll}
1 & 1 \\
1 & 0
\end{array}\right)^{-1}\right)
\end{array}
$$


from the left, and multiplying by

$$
\begin{gathered}
\operatorname{diag}\left(\operatorname{ad}\left(\begin{array}{ll}
1 & 1 \\
1 & 0
\end{array}\right) 1 \text { ad }\left(\begin{array}{ll}
1 & 1 \\
1 & 0
\end{array}\right) \operatorname{ad}\left(\begin{array}{ll}
1 & 1 \\
1 & 0
\end{array}\right) \operatorname{ad}\left(\begin{array}{cc}
x_{1} & 1 \\
1 & 0
\end{array}\right)\right. \\
\left.\operatorname{ad}\left(\begin{array}{cc}
x_{2} & 1 \\
1 & 0
\end{array}\right) \text { ad }\left(\begin{array}{ll}
1 & 1 \\
1 & 0
\end{array}\right) \text { ad }\left(\begin{array}{ll}
0 & 1 \\
1 & 0
\end{array}\right) \text { ad }\left(\begin{array}{ll}
1 & 1 \\
1 & 0
\end{array}\right)\right)
\end{gathered}
$$

from the right, where $\operatorname{diag}(\cdot)$ denotes the diagonal matrix whose diagonal entries are these entries. See Appendix B for a motivation of this modification. By the above modification, the modified $D_{1}$ has entries of the form

$$
\begin{aligned}
\operatorname{ad}\left(\begin{array}{ll}
1 & 1 \\
1 & 0
\end{array}\right)^{-1} \cdot\left(\mathcal{W}_{0}-1\right) & =\left(\begin{array}{cc|c}
-1 & 0 & -1 \\
1 & 2 & -1 \\
\hline 0 & 0 & 0
\end{array}\right), \\
\operatorname{ad}\left(\begin{array}{ll}
1 & 1 \\
1 & 0
\end{array}\right)^{-1} \cdot \mathcal{W}_{0} \cdot \operatorname{ad}\left(\begin{array}{ll}
1 & 1 \\
1 & 0
\end{array}\right) & =\left(\begin{array}{cc|c}
1 & -2 & -1 \\
0 & 1 & 1 \\
\hline 0 & 0 & 1
\end{array}\right), \\
\operatorname{ad}\left(\begin{array}{ll}
1 & 1 \\
1 & 0
\end{array}\right)^{-1} \cdot\left(-\mathcal{X}_{0}^{\prime-1} \mathcal{X}_{1}^{\prime}\right) \cdot \operatorname{ad}\left(\begin{array}{cc}
x_{1} & 1 \\
1 & 0
\end{array}\right) & =\left(\begin{array}{cc|c}
x_{1}-1 & 0 & 0 \\
0 & -1 & 0 \\
\hline 0 & 0 & \frac{1}{x_{1}-1}
\end{array}\right),
\end{aligned}
$$

with respect to the basis

$$
\left(\left(\begin{array}{ll}
0 & 1 \\
0 & 0
\end{array}\right),\left(\begin{array}{rr}
1 & 0 \\
0 & -1
\end{array}\right),\left(\begin{array}{ll}
0 & 0 \\
1 & 0
\end{array}\right)\right)
$$

of $\mathfrak{s l}_{2} \mathbb{C}$, and we can verify that any entry of the modified $D_{1}$ is of the form

$$
\left(\begin{array}{cc|c}
* & * & * \\
* & * & * \\
\hline 0 & 0 & *
\end{array}\right) .
$$

Further, we modify $D_{0}$ by multiplying by

$$
\begin{gathered}
\operatorname{diag}\left(\operatorname{ad}\left(\begin{array}{ll}
1 & 1 \\
1 & 0
\end{array}\right)^{-1} \quad 1 \quad \operatorname{ad}\left(\begin{array}{ll}
1 & 1 \\
1 & 0
\end{array}\right)^{-1} \operatorname{ad}\left(\begin{array}{ll}
1 & 1 \\
1 & 0
\end{array}\right)^{-1} \operatorname{ad}\left(\begin{array}{cc}
x_{1} & 1 \\
1 & 0
\end{array}\right)^{-1}\right. \\
\left.\operatorname{ad}\left(\begin{array}{cc}
x_{2} & 1 \\
1 & 0
\end{array}\right)^{-1} \operatorname{ad}\left(\begin{array}{ll}
1 & 1 \\
1 & 0
\end{array}\right)^{-1} \operatorname{ad}\left(\begin{array}{ll}
0 & 1 \\
1 & 0
\end{array}\right)^{-1} \operatorname{ad}\left(\begin{array}{ll}
1 & 1 \\
1 & 0
\end{array}\right)^{-1}\right)
\end{gathered}
$$


from the left. Then the modified $D_{0}$ has entries of the form

$$
\begin{aligned}
& \operatorname{ad}\left(\begin{array}{ll}
0 & 1 \\
1 & 0
\end{array}\right)^{-1} \cdot\left(\mathcal{X}_{3}-1\right)=\left(\begin{array}{crr}
* & * & 0 \\
1 / x_{2}-1 & 0 & 0 \\
\hline 0 & 0 & 0
\end{array}\right), \\
& \operatorname{ad}\left(\begin{array}{ll}
1 & 1 \\
1 & 0
\end{array}\right)^{-1} \cdot\left(\mathcal{Z}_{3}-1\right)=\left(\begin{array}{rrr}
-1 & -4 & 3 \\
-1 & -2 & 1 \\
\hline 0 & 0 & 0
\end{array}\right),
\end{aligned}
$$

and we can verify that any entry of the modified $D_{0}$ is of the form

$$
\left(\begin{array}{ccc}
* & * & * \\
* & * & * \\
\hline 0 & 0 & 0
\end{array}\right)
$$

We define $\widehat{C}^{1}$ to be the vector subspace of $C^{1}$ consisting of vectors of the form

$$
(* * 0|* * 0| \cdots|\cdots| * * 0)^{T} .
$$

We define $\widehat{C}^{2}$ to be the vector subspace of $C^{2}$ consisting of vectors of the form

$$
(* * 0|* * 0| \cdots \mid * * 0)^{T} .
$$

We set $\widehat{C}^{0}=C^{0}$. Since the modified $D_{0}$ and $D_{1}$ preserve these subspaces, $\widehat{C}^{*}$ forms a subcomplex of $C^{*}$ by these modified $D_{0}$ and $D_{1}$. We let $\widehat{D}_{0}$ and $\widehat{D}_{1}$ be the restrictions of these modified $D_{0}$ and $D_{1}$ to $\widehat{C}^{*}$.

We let $\check{C}^{*}=C^{*} / \widehat{C}^{*}$. By definition, $\check{C}^{0}=0$. We define $\check{D}_{1}$ to be the map on $\check{C}^{1}$ induced by the modified $D_{1}$ :

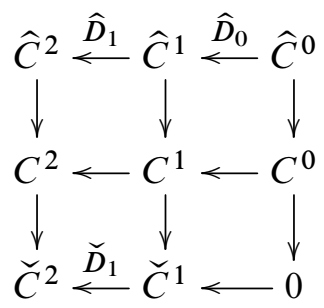

It is known that the calculation of the Reidemeister torsion of $C^{*}$ is reduced to the calculations of the Reidemeister torsions of $\widehat{C}^{*}$ and $\check{C}^{*}$,

$$
\tau\left(C^{*}\right)=\tau\left(\widehat{C}^{*}\right) \tau\left(\check{C}^{*}\right) .
$$


We can verify ${ }^{5}$ that $H^{2}\left(C^{*}\right) \cong H^{2}\left(\widehat{C}^{*}\right) \cong \mathbb{C}$ and $H^{1}\left(C^{*}\right) \cong H^{1}\left(\check{C}^{*}\right) \cong \mathbb{C}$ and the other cohomology groups of these cochain complexes vanish.

Step 4 In this step, we calculate the Reidemeister torsion of $\widehat{C}^{*}$.

We define the map $\widehat{D}_{2}: \widehat{C}^{2} \rightarrow \mathbb{C}$ to be the map evaluating 2-cochains by the cohomology class $\left[\partial E_{K}\right]$ of the boundary of the knot exterior $E_{K}$, where we choose the base point of $\partial E_{K}$ to be the base point of the 2-cell $r_{3}$. Then the following complex forms an acyclic complex,

$$
0 \longleftarrow \mathbb{C} \stackrel{\hat{D}_{2}}{\longleftarrow} \widehat{C}^{2} \stackrel{\hat{D}_{1}}{\longleftarrow} \widehat{C}^{1} \stackrel{\hat{D}_{0}}{\longleftarrow} \hat{C}^{0} \longleftarrow 0 .
$$

The Reidemeister torsion of $\widehat{C}^{*}$ is presented by

$$
\tau\left(\widehat{C}^{*}\right)=\left(\operatorname{det}\left(\widehat{E}_{1} \hat{D}_{0}\right) \operatorname{det}\left(\widehat{D}_{2} \widehat{E}_{2}\right) / \operatorname{det}\left(\hat{E}_{2} \widehat{D}_{1} \widehat{E}_{1}\right]\right),
$$

where we set

$$
\widehat{E}_{2}=\left(\begin{array}{c}
0 \\
0 \\
0 \\
0 \\
1 \\
0 \\
\vdots \\
0
\end{array}\right), \quad \widehat{E}_{1}=\left(\begin{array}{ccccc}
1 & & & & \\
& 1 & & & \\
& & \ddots & & \\
& & & 1 & \\
& & & 1 \\
& & & 0 \\
& & & 0 \\
& & & 0
\end{array}\right)
$$

We note that we can choose other $\widehat{E}_{2}$ and $\widehat{E}_{1}$ in such a way that the determinants of the defining formula of $\tau\left(\widehat{C}^{*}\right)$ are nonzero. It is known (see Turaev [27]) that the value of $\tau\left(\widehat{C}^{*}\right)$ does not depend on the choice of such $\widehat{E}_{2}$ and $\widehat{E}_{1}$.

${ }^{5}$ For any two-bridge knot, it is known (see Riley [22]) that the space of conjugacy classes of $\mathrm{PGL}_{2} \mathbb{C}$ representations of the knot group is 1-dimensional, and we can describe this space by a concrete polynomial (3). Since $H^{1}\left(C^{*}\right)$ is naturally isomorphic to the Zariski tangent space of this space, we can show (see Lemma D.1) that it is 1 -dimensional. It follows by counting dimensions of $C^{*}$ that $H^{2}\left(C^{*}\right)$ is also 1 -dimensional. Further, as mentioned above, we consider a subcomplex $\widehat{C}^{*}$ of $C^{*}$, and we set $\breve{C}^{*}=C^{*} / \widehat{C}^{*}$. By counting dimensions of these cochain groups, we can verify that $H^{2}\left(C^{*}\right) \cong H^{2}\left(\widehat{C}^{*}\right) \cong \mathbb{C}$ and $H^{1}\left(C^{*}\right) \cong H^{1}\left(\check{C}^{*}\right) \cong \mathbb{C}$ and the other cohomology groups of these cochain complexes vanish for any two-bridge knot. 
By definition, we have that

$$
\left.\operatorname{det}\left(\hat{E}_{1} \hat{D}_{0}\right]\right) \doteq \operatorname{det}\left(\begin{array}{c}
\text { the lowest } \\
\text { three rows } \\
\text { of } \hat{D}_{0}
\end{array}\right)=\operatorname{det}\left(\begin{array}{ccc}
\frac{1}{x_{2}}-1 & 0 & 0 \\
-1 & -4 & 3 \\
-1 & -2 & 1
\end{array}\right) \doteq 2\left(1-\frac{1}{x_{2}}\right),
$$

where " $\doteq "$ means that the left-hand side is equal to the right-hand side up to \pm 1 . For a general two-bridge knot, this value becomes $2\left(1-1 /\left(x_{m-1}\right)\right)$.

Further, we calculate $\operatorname{det}\left(\widehat{D}_{2} \widehat{E}_{2}\right)$, as follows. As mentioned above, $\widehat{D}_{2}$ is the map evaluating 2-cochains by $\left[\partial E_{K}\right]$ of the boundary $\partial E_{K}$ of the knot exterior $E_{K}$. We regard $K$ as a 1 -tangle in a 3-ball $B^{3}$. Then $\partial E_{K}$ consists of the boundary $\partial N(K)$ of a tubular neighborhood of $K$ and a $2-$ holed $\partial B^{3}$. Since $\partial N(K)$ is obtained by connecting 2-cells $r_{3}, r_{8}, r_{6}, \ldots$ in the form of a tube along the monodromy, the contribution of $\partial N(K)$ to $\hat{D}_{2}$ is given by

$$
\begin{aligned}
e_{r_{3}} & +\mathcal{X}_{0}^{\prime-1} \mathcal{X}_{1}^{-1} e_{r_{8}}+\mathcal{X}_{0}^{\prime-1} \mathcal{X}_{1}^{-1} \mathcal{Z}_{3}^{-1} \mathcal{X}_{3}^{-1} e_{r_{6}}+\mathcal{X}_{0}^{\prime-1} \mathcal{X}_{1}^{-1} \mathcal{Z}_{3}^{-1} \mathcal{X}_{3}^{-1} \mathcal{X}_{1}^{\prime-1} e_{r_{5}} \\
& -\mathcal{X}_{0}^{\prime-1} \mathcal{X}_{1}^{-1} \mathcal{Z}_{3}^{-1} \mathcal{X}_{3}^{-1} \mathcal{X}_{1}^{\prime-1} \mathcal{W}_{0}^{-1} e_{r_{1}}+\mathcal{X}_{0}^{\prime-1} \mathcal{X}_{1}^{-1} \mathcal{Z}_{3}^{-1} \mathcal{X}_{3}^{-1} \mathcal{X}_{1}^{\prime-1} \mathcal{W}_{0}{ }^{-1} e_{r_{2}} \\
& +\mathcal{X}_{0}^{\prime-1} \mathcal{X}_{1}^{-1} \mathcal{Z}_{3}^{-1} \mathcal{X}_{3}^{-1} \mathcal{X}_{1}^{\prime-1} \mathcal{W}_{0}^{-1} \mathcal{X}_{0}^{\prime-1} \mathcal{X}_{1}^{\prime} e_{r_{4}} \\
& +\mathcal{X}_{0}^{\prime-1} \mathcal{X}_{1}^{-1} \mathcal{Z}_{3}^{-1} \mathcal{X}_{3}^{-1} \mathcal{X}_{1}^{\prime-1} \mathcal{W}_{0}^{-1} \mathcal{X}_{0}^{\prime-1} \mathcal{X}_{1}^{\prime} \mathcal{X}_{2} e_{r_{7}} .
\end{aligned}
$$

Further, the contribution of a $2-$ holed $\partial B^{3}$ to $\widehat{D}_{2}$ is given by $-e_{r_{1}}+\left(e_{r_{2}}+\mathcal{W}_{0} e_{r_{3}}\right)+\mathcal{W}_{0}\left(\mathcal{X}_{1}^{-1} e_{r_{4}}+e_{r_{5}}\right)+\mathcal{W}_{0} \mathcal{X}_{1}^{-1}\left(e_{r_{6}}+\mathcal{X}_{2} e_{r_{7}}\right)+\mathcal{X}_{0}^{\prime-1} \mathcal{X}_{1}^{\prime} e_{r_{8}}$.

How to obtain this formula We consider the following 2-chains $r_{23}, r_{45}, r_{67}$ :
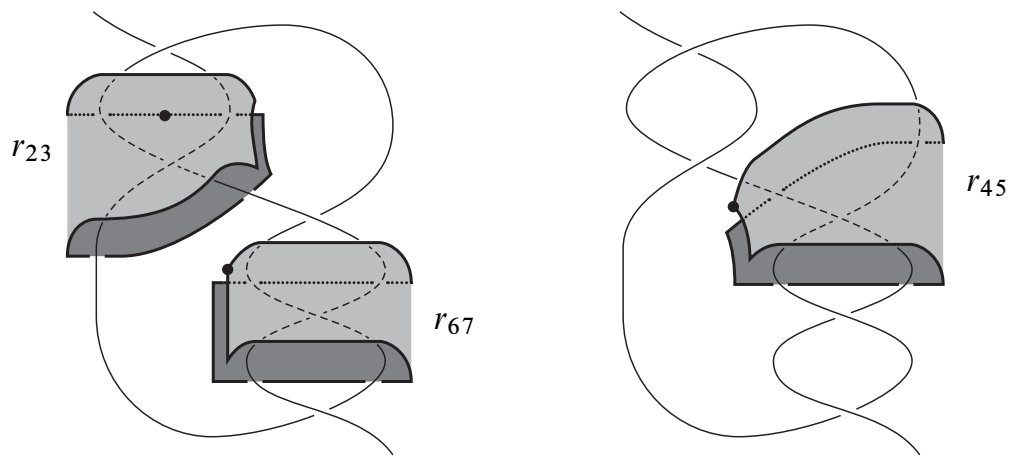

The relator around $r_{23}$ is $\mathcal{W}_{0} \mathcal{X}_{0} \mathcal{X}_{1}^{-1} \mathcal{W}_{1}^{-1} \mathcal{X}_{1}^{\prime-1} \mathcal{X}_{0}^{\prime}$, and its differential is given by

$$
e_{r_{23}}=e_{W_{0}}+\mathcal{W}_{0} e_{X_{0}}-\mathcal{X}_{0}^{\prime-1} \mathcal{X}_{1}^{\prime} e_{W_{1}}-\mathcal{X}_{0}^{\prime-1} \mathcal{X}_{1}^{\prime} \mathcal{W}_{1} e_{X_{1}}=e_{r_{2}}+\mathcal{W}_{0} e_{r_{3}} .
$$


Similarly, we can show that $e_{r_{45}}=e_{r_{4}}+\mathcal{X}_{1} e_{r_{5}}$ and $e_{r_{67}}=e_{r_{6}}+\mathcal{X}_{2} e_{r_{7}}$. The contribution of the 2 -holed $\partial B^{3}$ is obtained by connecting them along the monodromy,

$$
-e_{r_{1}}+e_{r_{23}}+\mathcal{W}_{0} \mathcal{X}_{1}^{-1} e_{r_{45}}+\mathcal{W}_{0} \mathcal{X}_{1}^{-1} e_{r_{67}}+\mathcal{X}_{0}^{\prime-1} \mathcal{X}_{1}^{\prime} e_{r_{8}}
$$

and this gives the aforementioned formula. Furthermore, recalling that the base point of $\partial E_{K}$ is the base point of $r_{3}$, the adjoint action of the meridian at this base point is given by

$$
\mathcal{X}_{0} \mathcal{X}_{0}^{\prime}=\operatorname{ad}\left(X_{0} X_{0}^{\prime}\right)=\operatorname{ad}\left(\begin{array}{cc}
1 & x_{1}-1 \\
0 & 1
\end{array}\right)=\left(\begin{array}{lll}
1 & * & * \\
0 & 1 & * \\
0 & 0 & 1
\end{array}\right)
$$

with respect to the basis (14) of $\mathfrak{s l}_{2} \mathbb{C}$, noting that we do not modify the basis vectors $e_{X_{0}}$ and $e_{r_{3}}$ in Step 3 unlike the other basis vectors. We choose $(001)$ as an invariant vector of this action, noting that we consider the action of multiplying the above matrix to a row vector from the right. Hence $D_{2}: C^{2} \rightarrow \mathbb{C}$ is represented by

$$
\begin{aligned}
D_{2}=\left(\begin{array}{lll}
0 & 0 & 1
\end{array}\right) \times\left(\left(\mathcal{X}_{0}^{\prime-1} \mathcal{X}_{1}^{-1} \mathcal{Z}_{3}^{-1} \mathcal{X}_{3}^{-1} \mathcal{X}_{1}^{\prime-1} \mathcal{W}_{0}{ }^{-1}\right.\right. \\
\left.\left.\mathcal{X}_{0}^{\prime-1} \mathcal{X}_{1}^{-1} \mathcal{Z}_{3}^{-1} \mathcal{X}_{3}^{-1} \mathcal{X}_{1}^{\prime-1} \mathcal{W}_{0}^{-1}, 1, \ldots\right)-\left(-1,1, \mathcal{W}_{0}, \ldots\right)\right)
\end{aligned}
$$

with respect to the basis $e_{r_{1}}, e_{r_{2}}, e_{r_{3}}, \ldots$ Further, $\widehat{D}_{2}$ is the restriction of the modified $D_{2}$ to $\widehat{C}^{2}$. Moreover, by definition, $\widehat{E}_{2}$ is given by

$$
\left(\begin{array}{l}
1 \\
0 \\
0
\end{array}\right)
$$

in the part of $e_{r_{3}} \in \mathfrak{s l}_{2} \mathbb{C}$ with respect to the basis (14) of $\mathfrak{s l}_{2} \mathbb{C}$. Hence only the $e_{r_{3}}$ part contributes to $\widehat{D}_{2} \widehat{E}_{2}$, and

$$
\widehat{D}_{2} \widehat{E}_{2}=\left(\begin{array}{lll}
0 & 0 & 1
\end{array}\right)\left(1-\mathcal{W}_{0}\right)\left(\begin{array}{l}
1 \\
0 \\
0
\end{array}\right)=\left(\begin{array}{lll}
0 & 0 & 1
\end{array}\right)\left(\begin{array}{ccc}
-3 & -4 & 1 \\
2 & 2 & 0 \\
1 & 0 & 1
\end{array}\right)\left(\begin{array}{l}
1 \\
0 \\
0
\end{array}\right)=1
$$

Therefore,

$$
\operatorname{det}\left(\widehat{D}_{2} \widehat{E}_{2}\right)=1 \text {. }
$$

We note that this holds for any two-bridge knot, since only the top 2-cell $r_{3}$ contributes to the resulting value, independently of the other part of the knot, as shown above. 
Hence we have that

$$
\tau\left(\hat{C}^{*}\right)=2\left(1-\frac{1}{x_{m-1}}\right) / \operatorname{det}\left(\hat{E}_{2} \hat{D}_{1} \widehat{E}_{1}\right) .
$$

Step 5 In this step, we calculate the Reidemeister torsion of $\check{C}^{*}$.

The Reidemeister torsion of $\check{C}^{*}$ is presented by

$$
\tau\left(\check{C}^{*}\right)=\operatorname{det}\left(h_{1} \check{E}_{1}\right] / \operatorname{det}\left(\check{D}_{1} \check{E}_{1}\right),
$$

where we set

$$
h_{1}=\left(\begin{array}{c}
* \\
1 \\
* \\
* \\
\vdots \\
*
\end{array}\right), \quad \check{E}_{1}=\left(\begin{array}{ccccc}
1 & & & & \\
0 & 0 & & & \\
& 1 & & & \\
& & 1 & & \\
& & & \ddots & \\
& & & & 1
\end{array}\right) \text {. }
$$

Here $h_{1}$ presents a cohomology class whose $e_{X_{0}}$ part in $C^{*}$ is

$$
\left(\begin{array}{l}
* \\
* \\
1
\end{array}\right) \text {. }
$$

We note that this class is invariant under the action of multiplying $\mathcal{X}_{0} \mathcal{X}_{0}^{\prime}$ from the left, and it is dual to the vector (001) which we used in the definition of $D_{2}$ in Step 4. By definition, we have that

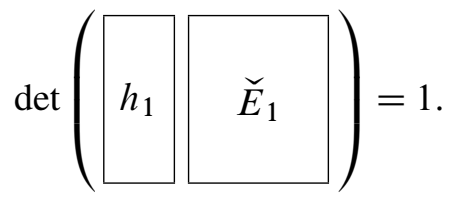

Hence

$$
\tau\left(\check{C}^{*}\right)=\frac{1}{\operatorname{det}\left(\check{D}_{1} \check{E}_{1}\right)}
$$


Step 6 By (15), (16) and (17), the Reidemeister torsion of $K$ is presented by

$$
\left.\frac{2}{\tau(K)}=\frac{1}{1-\frac{1}{x_{m-1}}} \operatorname{det}\left(\check{D}_{1} \check{E}_{1}\right) \operatorname{det}\left(\widehat{E}_{2} \widehat{D}_{1} \widehat{E}_{1}\right]\right)
$$

It is a problem to calculate the latter two factors in the right-hand side. We calculate them for any two-bridge knot in Sections 3.4 and 3.5.

\subsection{Decomposing two-bridge knot diagrams into elementary diagrams}

In this section, we decompose open two-bridge knot diagrams into elementary diagrams, and describe the hyperbolicity equations among parameters of such knot diagrams.

Any open two-bridge knots can be presented by a plat closure of a 3-braid of a product of copies of $\sigma_{1}$ and $\sigma_{2}^{-1}$, ie any open two-bridge knot diagram (or its mirror image) can be obtained by gluing copies of the following tangle diagrams, which we call elementary diagrams:
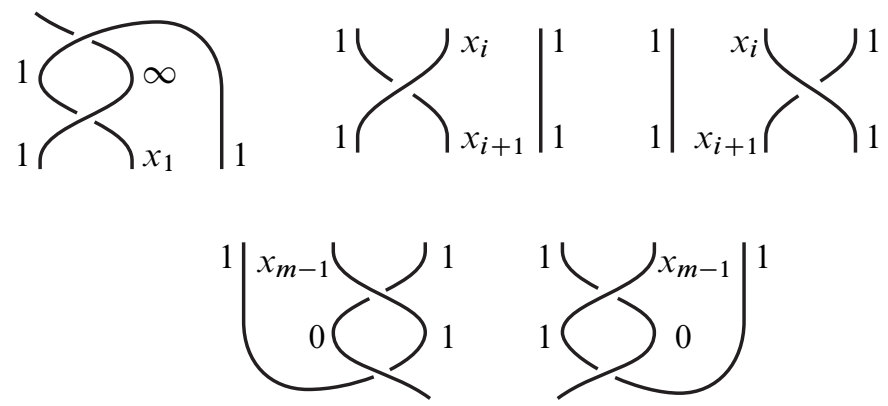

To describe the hyperbolicity equations among these parameters, we consider the parameters $\alpha_{i}$ and $\alpha_{i+1}$ at the ends of middle strands of $\sigma_{1}$ and $\sigma_{2}^{-1}$, as follows:

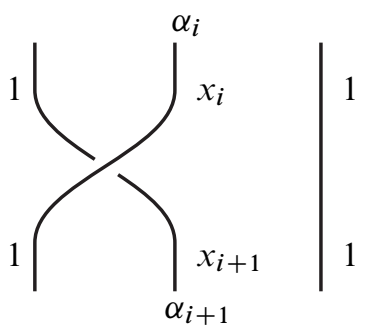

$$
\alpha_{i}=\frac{1-\frac{1}{x_{i}}}{1-\frac{x_{i+1}}{x_{i}}}, \quad \alpha_{i+1}=\frac{1-x_{i+1}}{1-\frac{x_{i+1}}{x_{i}}},
$$




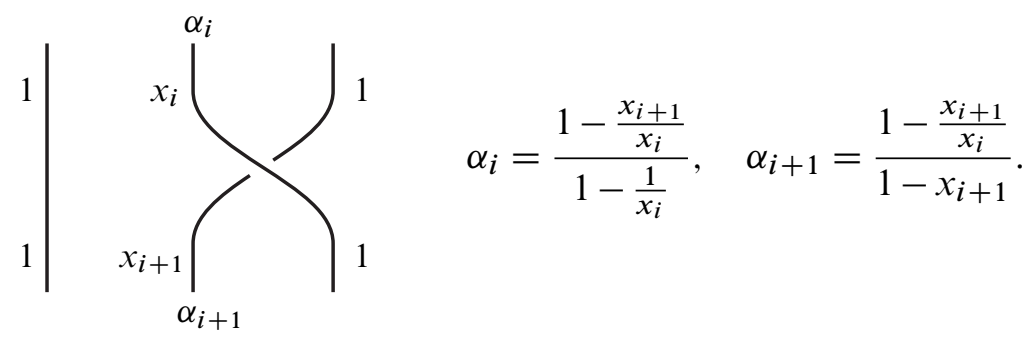

In general, for a parameterized tangle, we consider the parameter $\alpha$ at the end of a strand of the tangle diagram, as follows:

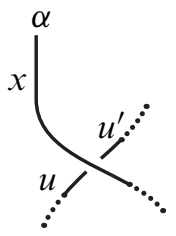

$$
\alpha=\frac{1-\frac{u}{x}}{1-\frac{u^{\prime}}{x}},
$$$$
\alpha=\frac{1-\frac{u}{x}}{1-\frac{u^{\prime}}{x}},
$$

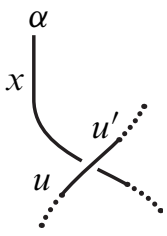

$$
\alpha=\frac{1-\frac{x}{u}}{1-\frac{x}{u^{\prime}}},
$$

$$
\alpha=\frac{1-\frac{x}{u}}{1-\frac{x}{u^{\prime}}} .
$$

When we glue two tangle diagrams, we require that these parameters coincide at each connecting point, which implies the hyperbolicity equation among parameters of the resulting tangle diagram.

\subsection{Calculation of $\operatorname{det}\left(\widehat{E}_{2} \mid \widehat{D}_{1} \widehat{E}_{1}\right)$}

In this section, we calculate

$$
\operatorname{det}\left(\hat{E}_{2} \widehat{D}_{1} \widehat{E}_{1}\right)
$$

for any two-bridge knot. We calculate it using a "representation" of parameterized 3-braids as we show in Lemma 3.1 below. The aim of this section is to prove this lemma.

As mentioned in Section 3.3, any open two-bridge knot can be obtained by gluing copies of elementary diagrams. 
Lemma 3.1 Let $D$ be a diagram of any open two-bridge knot, obtained by gluing copies of elementary diagrams. Then

$$
\operatorname{det}\left(\hat{E}_{2} \hat{D}_{1} \widehat{E}_{1}\right)=\Phi_{2}(D),
$$

where we define $\Phi_{2}(D)$ to be the composition of $\Phi_{2}$ of elementary diagrams whose values are given as follows:

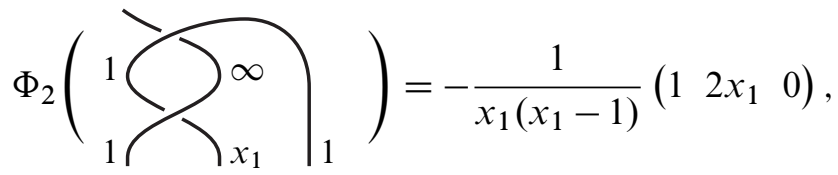

$$
\Phi_{2}\left(\left.\sum_{1}^{1} \int_{x_{i+1}}^{x_{i}}\right|_{1} ^{1}\right)=-\frac{1}{x_{i+1}}\left(\begin{array}{ccc}
1 & 2 x_{i+1} & 1 \\
0 & -x_{i+1} & -1 \\
0 & 0 & 1
\end{array}\right),
$$

$$
\Phi_{2}\left(\begin{array}{c|c}
1 & x_{i} \\
1 & x_{i+1}^{1}
\end{array}\right)=\frac{1}{1-\alpha_{i+1}}\left(\begin{array}{ccc}
1 & 0 & 0 \\
-1 & -x_{i+1} & 0 \\
1 & 2 x_{i+1} & 1
\end{array}\right),
$$
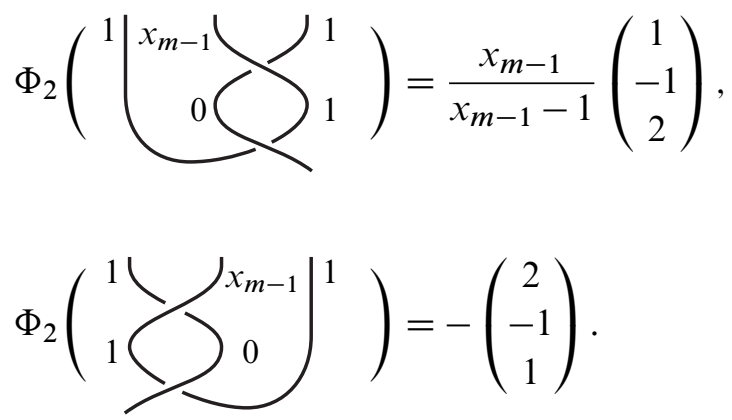

To prove Lemma 3.1, we consider the contribution of $\sigma_{1}$ to

$$
\operatorname{det}\left(\widehat{E}_{2} \widehat{D}_{1} \widehat{E}_{1}\right)
$$


given by:

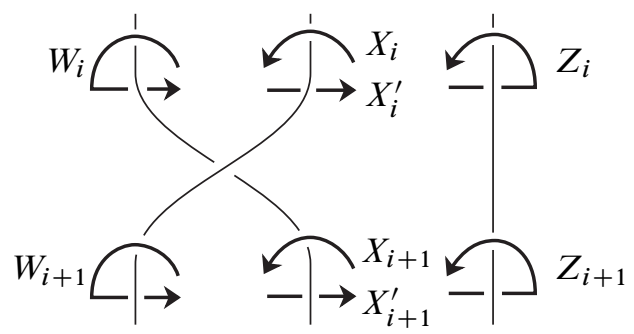

As explained in Section 3.1, we have that

$$
X_{i}^{\prime} \sim\left(\begin{array}{cc}
\frac{x_{i+1}}{x_{i}}-1 & 1-x_{i+1} \\
0 & \frac{1}{x_{i}}-1
\end{array}\right), \quad X_{i+1} \sim\left(\begin{array}{cc}
\frac{x_{i+1}}{x_{i}}-1 & 0 \\
\frac{1}{x_{i}}-1 & x_{i+1}-1
\end{array}\right)
$$

The relators among these matrices are given by

$$
W_{i} X_{i}^{\prime-1} X_{i+1}^{-1} X_{i+1}^{\prime}{ }^{-1} X_{i}^{\prime}, \quad X_{i} X_{i+1}^{-1} W_{i+1}^{-1} X_{i+1} X_{i}^{\prime}, \quad Z_{i} Z_{i+1}^{-1},
$$

and, as explained in Section 3.2, their differentials are given by

$$
\begin{aligned}
& e_{W_{i}}-\mathcal{X}_{i}^{\prime-1} \mathcal{X}_{i+1}^{\prime} e_{X_{i+1}} \\
& e_{X_{i}}-\mathcal{X}_{i}^{\prime-1} \mathcal{X}_{i+1}^{-1} e_{W_{i+1}}+\mathcal{X}_{i}^{\prime-1} \mathcal{X}_{i+1}^{-1}\left(1-\mathcal{W}_{i+1}\right) e_{X_{i+1}} \text {, } \\
& e_{Z_{i}}-e_{Z_{i+1}} \text {. }
\end{aligned}
$$

Hence, the corresponding part of $D_{1}$ is presented by

$$
D_{1}=\left(\begin{array}{ccc|ccc}
\ddots & \ddots & \ddots & 0 & 0 & 0 \\
\hline 1 & 0 & 0 & 0 & -\mathcal{X}_{i}^{\prime-1} \mathcal{X}_{i+1}^{\prime} & 0 \\
0 & 1 & 0 & -\mathcal{X}_{i}^{\prime-1} \mathcal{X}_{i+1}^{-1} & \mathcal{X}_{i}^{\prime-1} \mathcal{X}_{i+1}^{-1}\left(1-\mathcal{W}_{i+1}\right) & 0 \\
0 & 0 & 1 & 0 & 0 & -1 \\
\hline 0 & 0 & 0 & \ddots & \ddots & \ddots
\end{array}\right)
$$

with respect to the basis $\left(e_{W_{i}}, e_{X_{i}}, e_{Z_{i}}, e_{W_{i+1}}, e_{X_{i+1}}, e_{Z_{i+1}}\right)$. We consider to calculate the determinant of a matrix of the following form for a given value of $\left(A_{i} B_{i} C_{i}\right)$,

$$
\left(\begin{array}{ccc|ccc}
A_{i} & B_{i} & C_{i} & 0 & 0 & 0 \\
\hline 1 & 0 & 0 & 0 & -\mathcal{X}_{i}^{\prime-1} \mathcal{X}_{i+1}^{\prime} & 0 \\
0 & 1 & 0 & -\mathcal{X}_{i}^{\prime-1} \mathcal{X}_{i+1}^{-1} & \mathcal{X}_{i}^{\prime-1} \mathcal{X}_{i+1}^{-1}\left(1-\mathcal{W}_{i+1}\right) & 0 \\
0 & 0 & 1 & 0 & 0 & -1 \\
\hline 0 & 0 & 0 & \ddots & \ddots & \ddots
\end{array}\right),
$$


by modifying it by elementary transformations (where "elementary transformations" include suppression of rows and columns), noting that elementary transformations do not change the determinant. That is, the strategy is to show that (26) is related to the form below by elementary transformations, and, when the value of $\left(A_{i} B_{i} C_{i}\right)$ is given, the value of $\left(A_{i+1} B_{i+1} C_{i+1}\right)$ of the following formula can be obtained from the value of $\left(A_{i} B_{i} C_{i}\right)$, which enables us to calculate the required determinant recursively. The matrix (26) can be modified by elementary transformations as follows:

$$
\begin{aligned}
& \text { (26) } \approx\left(\begin{array}{ccc|cccc}
1 & 0 & 0 & 0 & -\mathcal{X}_{i}^{\prime-1} \mathcal{X}_{i+1}^{\prime} & 0 \\
0 & 1 & 0 & -\mathcal{X}_{i}^{\prime-1} \mathcal{X}_{i+1}^{-1} & \mathcal{X}_{i}^{\prime-1} \mathcal{X}_{i+1}^{-1}\left(1-\mathcal{W}_{i+1}\right) & 0 \\
0 & 0 & 1 & 0 & 0 & -1 \\
\hline A_{i} & B_{i} & C_{i} & 0 & 0 & 0 \\
\hline 0 & 0 & 0 & \ddots & \ddots & \ddots
\end{array}\right) \\
& \approx\left(\begin{array}{ccc|ccc}
1 & 0 & 0 & 0 & -\mathcal{X}_{i}^{\prime-1} \mathcal{X}_{i+1}^{\prime} & 0 \\
0 & 1 & 0 & -\mathcal{X}_{i}^{\prime-1} \mathcal{X}_{i+1}^{-1} & \mathcal{X}_{i}^{\prime-1} \mathcal{X}_{i+1}^{-1}\left(1-\mathcal{W}_{i+1}\right) & 0 \\
0 & 0 & 1 & 0 & 0 & -1 \\
\hline 0 & 0 & 0 & A_{i+1} & B_{i+1} & C_{i+1} \\
\hline 0 & 0 & 0 & \ddots & \ddots & \ddots
\end{array}\right) \\
& \approx\left(\begin{array}{ccc}
A_{i+1} & B_{i+1} & C_{i+1} \\
\hline \ddots & \ddots & \ddots
\end{array}\right),
\end{aligned}
$$

where " $\approx$ " means that the matrices of both sides are related by elementary transformations (hence they have equal determinants), and we set

$$
\left(\begin{array}{lll}
A_{i+1} & B_{i+1} & C_{i+1}
\end{array}\right)=\left(\begin{array}{lll}
A_{i} & B_{i} & C_{i}
\end{array}\right)\left(\begin{array}{ccc}
0 & \mathcal{X}_{i}^{\prime-1} \mathcal{X}_{i+1}^{\prime} & 0 \\
\mathcal{X}_{i}^{\prime-1} \mathcal{X}_{i+1}^{-1} & \mathcal{X}_{i}^{\prime-1} \mathcal{X}_{i+1}^{-1}\left(\mathcal{W}_{i+1}-1\right) & 0 \\
0 & 0 & 1
\end{array}\right)
$$

Hence, $\sigma_{1}$ is taken by the "representation" by

$$
\left.{ }_{1}^{1} \int_{x_{i+1}}^{x_{i}}\right|_{1} ^{1} \longmapsto\left(\begin{array}{ccc}
0 & \mathcal{X}_{i}^{\prime-1} \mathcal{X}_{i+1}^{\prime} & 0 \\
\mathcal{X}_{i}^{\prime-1} \mathcal{X}_{i+1}^{-1} & \mathcal{X}_{i}^{\prime-1} \mathcal{X}_{i+1}^{-1}\left(\mathcal{W}_{i+1}-1\right) & 0 \\
0 & 0 & 1
\end{array}\right) .
$$


We consider the subcomplex $\widehat{C}^{*}$ as in Section 3.2, and consider the matrix that corresponds to the one of (27). We multiply the matrix of (27) by

$$
\operatorname{diag}\left(\operatorname{ad}\left(\begin{array}{ll}
1 & 1 \\
1 & 0
\end{array}\right)^{-1} \operatorname{ad}\left(\begin{array}{cc}
x_{i} & 1 \\
1 & 0
\end{array}\right)^{-1} \operatorname{ad}\left(\begin{array}{ll}
1 & 1 \\
1 & 0
\end{array}\right)^{-1}\right)
$$

from the left, and by

$$
\operatorname{diag}\left(\operatorname{ad}\left(\begin{array}{ll}
1 & 1 \\
1 & 0
\end{array}\right) \operatorname{ad}\left(\begin{array}{cc}
x_{i+1} & 1 \\
1 & 0
\end{array}\right) \operatorname{ad}\left(\begin{array}{ll}
1 & 1 \\
1 & 0
\end{array}\right)\right)
$$

from the right. Then the entries of the resulting matrix are presented by

$$
\begin{gathered}
\operatorname{ad}\left(\begin{array}{ll}
1 & 1 \\
1 & 0
\end{array}\right)^{-1} \cdot \mathcal{X}_{i}^{\prime-1} \mathcal{X}_{i+1}^{\prime} \cdot \operatorname{ad}\left(\begin{array}{cc}
x_{i+1} & 1 \\
1 & 0
\end{array}\right)=\left(\begin{array}{cc|c}
-\frac{x_{i}\left(x_{i+1}-1\right)}{x_{i}-1} & 0 & 0 \\
0 & 1 & 0 \\
\hline 0 & 0 & -\frac{x_{i}-1}{x_{i}\left(x_{i+1}-1\right)}
\end{array}\right) \\
\operatorname{ad}\left(\begin{array}{cc}
x_{i} & 1 \\
1 & 0
\end{array}\right)^{-1} \cdot \mathcal{X}_{i}^{\prime-1} \mathcal{X}_{i+1}^{-1} \cdot \operatorname{ad}\left(\begin{array}{ll}
1 & 1 \\
1 & 0
\end{array}\right)=\left(\begin{array}{cc|c}
-\frac{x_{i+1}-1}{\left(x_{i}-1\right) x_{i}} & \frac{2}{x_{i}} & * \\
0 & 1 & * \\
\hline 0 & 0 & -\frac{\left(x_{i}-1\right) x_{i}}{x_{i+1}-1}
\end{array}\right) \\
\operatorname{ad}\left(\begin{array}{cc}
x_{i} & 1 \\
1 & 0
\end{array}\right)^{-1} \cdot \mathcal{X}_{i}^{\prime-1} \mathcal{X}_{i+1}^{-1}\left(\mathcal{W}_{i+1}-1\right) \cdot \operatorname{ad}\left(\begin{array}{cc}
x_{i+1} & 1 \\
1 & 0
\end{array}\right) \\
=\left(\begin{array}{ccc}
-\frac{\left(x_{i+1}-1\right)^{2}\left(2 x_{i}+x_{i+1}-1\right)}{\left(x_{i}-1\right) x_{i}} & \frac{2\left(x_{i+1}-1\right)\left(2 x_{i}+x_{i+1}-2\right)}{\left(x_{i}-1\right) x_{i}} \\
-\left(x_{i+1}-1\right)^{2} & 2\left(x_{i+1}-1\right) & * \\
0 & 0 & 0
\end{array}\right)
\end{gathered}
$$

Hence the restriction of the matrix of (27) to the subcomplex $\widehat{C}^{*}$ is presented by

$$
\left(\begin{array}{cc|cc|cc}
0 & 0 & -\frac{x_{i}\left(x_{i+1}-1\right)}{x_{i}-1} & 0 & 0 & 0 \\
0 & 0 & 0 & 1 & 0 & 0 \\
\hline-\frac{x_{i+1}-1}{\left(x_{i}-1\right) x_{i}} & \frac{2}{x_{i}} & -\frac{\left(x_{i+1}-1\right)^{2}\left(2 x_{i}+x_{i+1}-1\right)}{\left(x_{i}-1\right) x_{i}} & \frac{2\left(x_{i+1}-1\right)\left(2 x_{i}+x_{i+1}-2\right)}{\left(x_{i}-1\right) x_{i}} & 0 & 0 \\
0 & 1 & -\left(x_{i+1}-1\right)^{2} & 2\left(x_{i+1}-1\right) & 0 & 0 \\
\hline 0 & 0 & 0 & 0 & 1 & 0 \\
0 & 0 & 0 & 0 & 0 & 1
\end{array}\right)
$$


We consider the contribution of $\sigma_{2}^{-1}$ to

$$
\operatorname{det}\left(\hat{E}_{2} \hat{D}_{1} \widehat{E}_{1}\right) \text {. }
$$

given by:

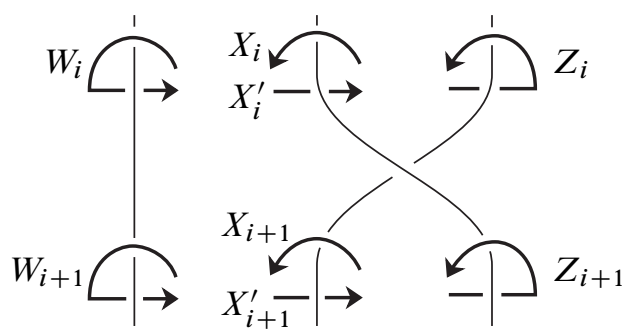

As explained in Section 3.1, we have that

$$
X_{i}^{\prime} \sim\left(\begin{array}{cc}
\frac{1}{x_{i}}-1 & x_{i+1}-1 \\
0 & \frac{x_{i+1}}{x_{i}}-1
\end{array}\right), \quad X_{i+1} \sim\left(\begin{array}{cc}
x_{i+1}-1 & 0 \\
1-\frac{1}{x_{i}} & \frac{x_{i+1}}{x_{i}}-1
\end{array}\right) .
$$

The relators among these matrices are given by

$$
W_{i} W_{i+1}^{-1}, \quad X_{i} X_{i}^{\prime} X_{i+1} Z_{i+1}^{-1} X_{i+1}^{-1}, \quad Z_{i} X_{i}^{\prime} X_{i+1}^{\prime}{ }^{-1} X_{i+1}^{-1} X_{i}^{\prime-1} .
$$

Hence, similarly as the case of $\sigma_{1}$, the corresponding part of $D_{1}$ is presented by

$$
D_{1}=\left(\begin{array}{ccc|ccc}
\ddots & \ddots & \ddots & 0 & 0 & 0 \\
\hline 1 & 0 & 0 & -1 & 0 & 0 \\
0 & 1 & 0 & 0 & \mathcal{X}_{i} \mathcal{X}_{i}^{\prime}-1 & -\mathcal{X}_{i+1} \\
0 & 0 & 1 & 0 & -\mathcal{X}_{i}^{\prime} & 0 \\
\hline 0 & 0 & 0 & \ddots & \ddots & \ddots
\end{array}\right)
$$

with respect to the basis $\left(e_{W_{i}}, e_{X_{i}}, e_{Z_{i}}, e_{W_{i+1}}, e_{X_{i+1}}, e_{Z_{i+1}}\right)$. Further, $\sigma_{2}^{-1}$ is taken by the "representation" by

$$
\left.{ }_{1}^{1}\right|_{x_{i+1}}{ }_{1}^{x_{i}} \longmapsto\left(\begin{array}{ccc}
1 & 0 & 0 \\
0 & 1-\mathcal{X}_{i} \mathcal{X}_{i}^{\prime} & \mathcal{X}_{i+1} \\
0 & \mathcal{X}_{i}^{\prime} & 0
\end{array}\right) \text {. }
$$

We consider its restriction to the subcomplex $\widehat{C}^{*}$, similarly as the case of $\sigma_{1}$. We multiply the matrix of (29) by

$$
\operatorname{diag}\left(\operatorname{ad}\left(\begin{array}{ll}
1 & 1 \\
1 & 0
\end{array}\right)^{-1} \operatorname{ad}\left(\begin{array}{cc}
x_{i} & 1 \\
1 & 0
\end{array}\right)^{-1} \operatorname{ad}\left(\begin{array}{ll}
1 & 1 \\
1 & 0
\end{array}\right)^{-1}\right)
$$


from the left, and multiply by

$$
\operatorname{diag}\left(\operatorname{ad}\left(\begin{array}{ll}
1 & 1 \\
1 & 0
\end{array}\right) \text { ad }\left(\begin{array}{cc}
x_{i+1} & 1 \\
1 & 0
\end{array}\right) \operatorname{ad}\left(\begin{array}{ll}
1 & 1 \\
1 & 0
\end{array}\right)\right)
$$

from the right. Then the entries of the resulting matrix are presented by $\operatorname{ad}\left(\begin{array}{cc}x_{i} & 1 \\ 1 & 0\end{array}\right)^{-1} \cdot\left(1-\mathcal{X}_{i} \mathcal{X}_{i}^{\prime}\right) \cdot \operatorname{ad}\left(\begin{array}{cc}x_{i+1} & 1 \\ 1 & 0\end{array}\right)$

$$
\begin{array}{r}
=\left(\begin{array}{cc|c}
\frac{\left(2 x_{i}-x_{i+1}+1\right)\left(x_{i+1}-1\right)}{x_{i}^{2}} & \frac{2\left(x_{i}-x_{i+1}+1\right)\left(x_{i+1}-1\right)}{x_{i}^{2}\left(x_{i}-x_{i+1}\right)} \\
\frac{\left(x_{i}-x_{i+1}\right)\left(x_{i+1}-1\right)}{x_{i}} & \frac{2\left(x_{i+1}-1\right)}{x_{i}} & * \\
0 & 0 & *
\end{array}\right), \\
\operatorname{ad}\left(\begin{array}{cc}
x_{i} & 1 \\
1 & 0
\end{array}\right)^{-1} \cdot \mathcal{X}_{i+1} \cdot \operatorname{ad}\left(\begin{array}{ll}
1 & 1 \\
1 & 0
\end{array}\right)=\left(\begin{array}{cc|c}
-\frac{x_{i+1}-1}{x_{i}\left(x_{i}-x_{i+1}\right)} & \frac{2\left(x_{i}-1\right)}{x_{i}\left(x_{i}-x_{i+1}\right)} & * \\
0 & 1 & * \\
\hline 0 & 0 & \frac{x_{i}\left(x_{i+1}-x_{i}\right)}{x_{i+1}-1}
\end{array}\right), \\
\operatorname{ad}\left(\begin{array}{ll}
1 & 1 \\
1 & 0
\end{array}\right)^{-1} \cdot \mathcal{X}_{i}^{\prime} \cdot \operatorname{ad}\left(\begin{array}{cc}
x_{i+1} & 1 \\
1 & 0
\end{array}\right)=\left(\begin{array}{cc|c}
\frac{x_{i}-x_{i+1}}{x_{i}-1} & 0 & 0 \\
0 & 1 & 0 \\
\hline 0 & 0 & \frac{x_{i}-1}{x_{i}-x_{i+1}}
\end{array}\right) .
\end{array}
$$

Hence the restriction of the matrix of (29) to the subcomplex $\widehat{C}^{*}$ is presented by:

(30)

$$
\left(\begin{array}{cc|cc|cc}
1 & 0 & 0 & 0 & 0 & 0 \\
0 & 1 & 0 & 0 & 0 & 0 \\
\hline 0 & 0 & \frac{\left(2 x_{i}-x_{i+1}+1\right)\left(x_{i+1}-1\right)}{x_{i}^{2}} & \frac{2\left(x_{i}-x_{i+1}+1\right)\left(x_{i+1}-1\right)}{x_{i}^{2}\left(x_{i}-x_{i+1}\right)} & -\frac{x_{i+1}-1}{x_{i}\left(x_{i}-x_{i}+1\right)} & \frac{2\left(x_{i}-1\right)}{x_{i}\left(x_{i}-x_{i+1}\right)} \\
0 & 0 & \frac{\left(x_{i}-x_{i+1}\right)\left(x_{i+1}-1\right)}{x_{i}} & \frac{2\left(x_{i+1}-1\right)}{x_{i}} & 0 & 1 \\
\hline 0 & 0 & \frac{x_{i}-x_{i+1}}{x_{i}-1} & 0 & 0 & 0 \\
0 & 0 & 0 & 1 & 0 & 0
\end{array}\right)
$$

The matrices (28) and (30) give a 6-dimensional "representation" of parameterized 3braids. In fact, as we show in the proof of Lemma 3.1 below, only a 3-dimensional subspace contributes to the calculation of the required value. A basis of this 3-dimensional subspace is given by

$$
\begin{aligned}
& e_{1}=\left(\begin{array}{llll}
0 & 0 & -\alpha_{i}\left(x_{i}-1\right) x_{i} & 1-\alpha_{i}+x_{i}+\alpha_{i} x_{i}-\left(\alpha_{i}-1\right)\left(x_{i}-1\right)\left(\alpha_{i}-1\right)\left(x_{i}-3\right)
\end{array}\right),
\end{aligned}
$$

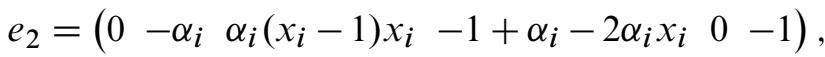

$$
\begin{aligned}
& e_{3}=\left(\left(\alpha_{i}-1\right)\left(x_{i}-1\right)\left(\alpha_{i}-1\right)\left(x_{i}+1\right)-\alpha_{i}\left(x_{i}-1\right) x_{i} 1-\alpha_{i}-x_{i}+3 \alpha_{i} x_{i} \quad 0 \quad 0\right) \text {. }
\end{aligned}
$$


Remark 3.2 We explain how to obtain this basis concretely, though we give a proof of Lemma 3.1 below without using this remark: $\left(\sigma_{1} \sigma_{2}\right)^{3}$ is a central element of the 3 -braid group. For any open two-bridge knot, when we insert $\left(\sigma_{1} \sigma_{2}\right)^{3}$ at any place of a 3-braid, the knot type of the open two-bridge knot does not change. Further, the values of $x_{i}$ and $\alpha_{i}$ are invariant under the action of $\left(\sigma_{1} \sigma_{2}\right)^{3}$. Hence, only the 1 -eigenspace with respect to the action of $\left(\sigma_{1} \sigma_{2}\right)^{3}$ contributes to the required value. Let $M$ be the matrix of the action of $\left(\sigma_{1} \sigma_{2}\right)^{3}$. A concrete calculation shows $M$ has eigenvalue 1 of multiplicity 6 . Let $M^{\prime}$ be $M$ - (identity matrix). The 1-eigenspace is the kernel of the multiplication of $M^{\prime}$ from the right, noting that we consider an "eigenspace" as an eigenspace with respect to the action of multiplying matrices to row vectors from the right. We consider the vector space spanned by the column vectors of $M^{\prime}$. We can show by concrete calculation that this vector space is the 3-dimensional vector space spanned by

$$
\begin{aligned}
& \left(\begin{array}{lllll}
1 & 0 & -\frac{1-\alpha_{i}+2 \alpha_{i} x_{i}}{\alpha_{i} x_{i}^{2}}-\frac{x_{i}-1}{x_{i}} & 1 & 0
\end{array}\right)^{T},
\end{aligned}
$$

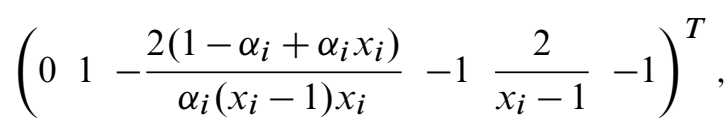

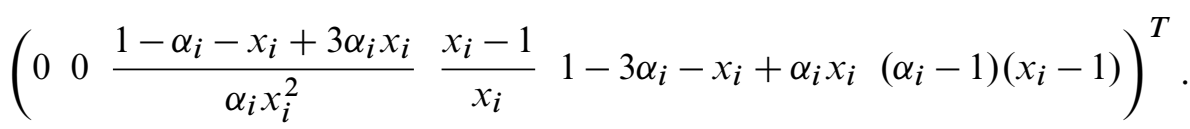

Hence, we can show by concrete calculation that the 1-eigenspace is the 3-dimensional vector space spanned by $e_{1}, e_{2}$ and $e_{3}$. In this 1 -eigenspace, we obtain $e_{3}$ as an eigenvector of the action of $\sigma_{1}$, we obtain $e_{1}$ as an eigenvector of the action of $\sigma_{2}$, and we obtain $e_{2}$ as an eigenvector of the action of $\sigma_{1} \sigma_{2} \sigma_{1}$.

Proof of Lemma 3.1 We can show by concrete calculation that the matrices (28) and (30) are rewritten as the matrices (21) and (22) with respect to the basis $\left(e_{1}, e_{2}, e_{3}\right)$. By these matrices, we define a "representation" $\Phi_{2}$ of parameterized 3-braids, as we mention in the statement of the lemma. In particular, this 3-dimensional space is preserved by the actions of $\sigma_{1}$ and $\sigma_{2}$. Further, we will show below that the vector corresponding to the elementary diagram of the top part belongs to this 3-dimensional space. Hence, only this 3 -dimensional space contributes to the required value of the lemma.

In the following, we calculate the vectors of (20), (23) and (24), which are contributions from elementary diagrams of the top and bottom parts to the required value. 
Calculation of (20) We calculate the contribution from the top part of an open twobridge knot to the required value, as follows:
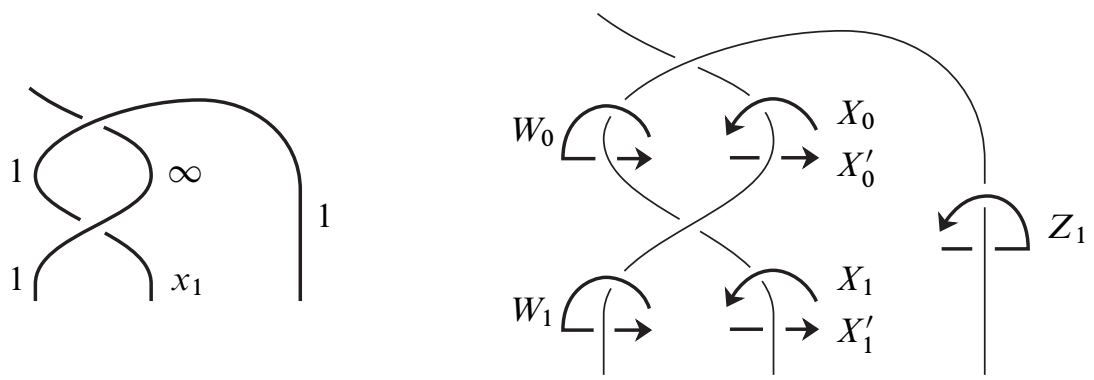

As explained in Section 3.1, we have that

$$
X_{0} \sim\left(\begin{array}{ll}
1 & 0 \\
0 & 1
\end{array}\right), \quad X_{0}^{\prime} \sim\left(\begin{array}{cc}
1 & x_{1}-1 \\
0 & 1
\end{array}\right), \quad X_{1} \sim\left(\begin{array}{cc}
1 & 0 \\
1 & 1-x_{1}
\end{array}\right), \quad X_{1}^{\prime} \sim\left(\begin{array}{cc}
\frac{1}{x_{1}} & -x_{1} \\
0 & \frac{1}{x_{1}}-1
\end{array}\right) .
$$

As explained in Section 3.2, the corresponding part of $D_{1}$ is presented by

$$
\left(\begin{array}{cc|ccc}
1 & \mathcal{W}_{0}-1 & 0 & 0 & \mathcal{Z}_{1}^{-1} \\
\hline 1 & 0 & 0 & -\mathcal{X}_{0}^{\prime-1} \mathcal{X}_{1}^{\prime} & 0 \\
0 & 1 & -\mathcal{X}_{0}^{\prime-1} \mathcal{X}_{1}^{-1} & \mathcal{X}_{0}^{\prime-1} \mathcal{X}_{1}^{-1}\left(1-\mathcal{W}_{1}\right) & 0 \\
\hline & & \ddots & \ddots & \ddots
\end{array}\right)
$$

with respect to the basis $\left(e_{W_{0}}, e_{X_{0}}, e_{W_{1}}, e_{X_{1}}, e_{Z_{1}}\right)$. We multiply the above matrix by

$$
\operatorname{diag}\left(\operatorname{ad}\left(\begin{array}{ll}
1 & 1 \\
1 & 0
\end{array}\right)^{-1} \operatorname{ad}\left(\begin{array}{ll}
1 & 1 \\
1 & 0
\end{array}\right)^{-1} \quad 1\right)
$$

from the left, and by

$$
\operatorname{diag}\left(\operatorname{ad}\left(\begin{array}{ll}
1 & 1 \\
1 & 0
\end{array}\right) \quad 1 \quad \operatorname{ad}\left(\begin{array}{ll}
1 & 1 \\
1 & 0
\end{array}\right) \text { ad }\left(\begin{array}{cc}
x_{1} & 1 \\
1 & 0
\end{array}\right) \text { ad }\left(\begin{array}{ll}
1 & 1 \\
1 & 0
\end{array}\right)\right)
$$

from the right. Then the entries of the resulting matrix are presented by

$$
\begin{aligned}
\operatorname{ad}\left(\begin{array}{ll}
1 & 1 \\
1 & 0
\end{array}\right)^{-1} \cdot\left(\mathcal{W}_{0}-1\right) & =\left(\begin{array}{cc|c}
-1 & 0 & -1 \\
1 & 2 & -1 \\
\hline 0 & 0 & 0
\end{array}\right), \\
\operatorname{ad}\left(\begin{array}{ll}
1 & 1 \\
1 & 0
\end{array}\right)^{-1} \cdot \mathcal{Z}_{1}^{-1} \cdot \operatorname{ad}\left(\begin{array}{ll}
1 & 1 \\
1 & 0
\end{array}\right) & =\left(\begin{array}{cc|c}
1 & -2 & -1 \\
0 & 1 & 1 \\
\hline 0 & 0 & 1
\end{array}\right),
\end{aligned}
$$




$$
\begin{aligned}
\operatorname{ad}\left(\begin{array}{ll}
1 & 1 \\
1 & 0
\end{array}\right)^{-1} \cdot\left(-\mathcal{X}_{0}^{\prime-1} \mathcal{X}_{1}^{\prime}\right) \cdot \operatorname{ad}\left(\begin{array}{cc}
x_{1} & 1 \\
1 & 0
\end{array}\right) & =\left(\begin{array}{cc|c}
x_{1}-1 & 0 & 0 \\
0 & -1 & 0 \\
\hline 0 & 0 & \frac{1}{x_{1}-1}
\end{array}\right) \\
\left(-\mathcal{X}_{0}^{\prime-1} \mathcal{X}_{1}^{-1}\right) \cdot \operatorname{ad}\left(\begin{array}{ll}
1 & 1 \\
1 & 0
\end{array}\right) & =\left(\begin{array}{cc|c}
1-x_{1} & 0 & 0 \\
0 & -1 & 0 \\
\hline 0 & 0 & \frac{1}{1-x_{1}}
\end{array}\right) \\
\mathcal{X}_{0}^{\prime-1} \mathcal{X}_{1}^{-1}\left(1-\mathcal{W}_{1}\right) \cdot \operatorname{ad}\left(\begin{array}{cc}
x_{1} & 1 \\
1 & 0
\end{array}\right) & =\left(\begin{array}{cc|c}
* & * & x_{1}-1 \\
\left.\hline x_{1}-1\right)^{2} & 2\left(1-x_{1}\right) & -1 \\
\hline 0 & 0 & 0
\end{array}\right) .
\end{aligned}
$$

Hence, the restriction of the matrix (31) to the subcomplex $\widehat{C}^{*}$ is presented by

$$
\left(\begin{array}{cc|cc|cc|cc|cc}
1 & 0 & -1 & 0 & 0 & 0 & 0 & 0 & 1 & -2 \\
0 & 1 & 1 & 2 & 0 & 0 & 0 & 0 & 0 & 1 \\
\hline 1 & 0 & 0 & 0 & 0 & 0 & x_{1}-1 & 0 & 0 & 0 \\
0 & 1 & 0 & 0 & 0 & 0 & 0 & -1 & 0 & 0 \\
\hline 0 & 0 & 1 & 0 & 1-x_{1} & 0 & * & * & 0 & 0 \\
0 & 0 & 0 & 1 & 0 & -1 & \left(x_{1}-1\right)^{2} & 2\left(1-x_{1}\right) & 0 & 0 \\
\hline & & & \ddots & \ddots & \ddots & \ddots & \ddots & \ddots
\end{array}\right) .
$$

When we calculate

$$
\operatorname{det}\left(\widehat{E}_{2} \widehat{D}_{1} \widehat{E}_{1}\right),
$$

we remove the fifth row of $\widehat{D}_{1}$ from the definition of $\widehat{E}_{2}$. The matrix obtained from the above matrix by removing the fifth row is equivalent to the following matrix by elementary transformations:

$$
\left(\begin{array}{cc|cc|cc}
0 & -2 & 2 x_{1}^{2}-3 x_{1}+1 & -4 x_{1}+3 & -1 & 1 \\
\hline \ddots & \ddots & \ddots & \ddots & \ddots & \ddots
\end{array}\right) .
$$

Further, the vector

$$
\left(0-22 x_{1}^{2}-3 x_{1}+1-4 x_{1}+3-1 \quad 1\right)
$$

belongs to the vector space spanned by $e_{1}, e_{2}, e_{3}$, and it is rewritten as the vector (20) with respect to the basis $\left(e_{1}, e_{2}, e_{3}\right)$. Hence, we define $\Phi_{2}$ of the top part of an open two-bridge knot by (20). 
Calculation of (23) We calculate the contribution from a bottom part of an open two-bridge knot to the required value as follows:
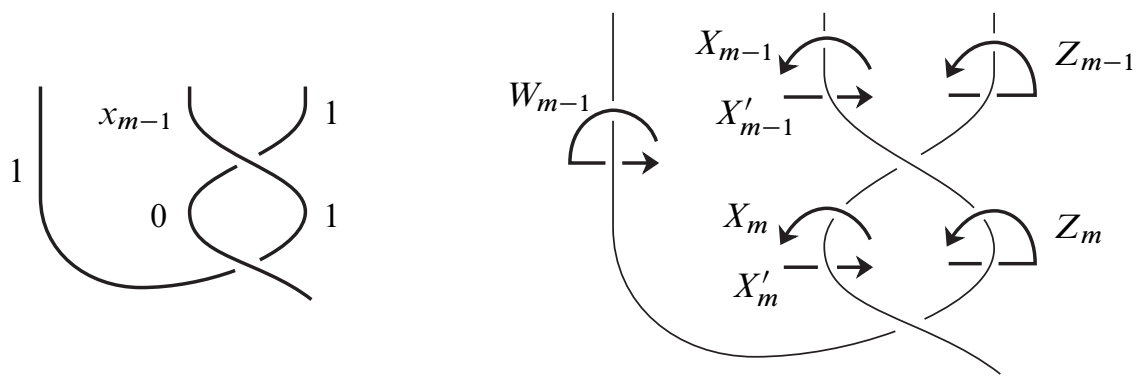

As explained in Section 3.1, we have that

$$
\begin{gathered}
X_{m-1} \sim\left(\begin{array}{cc}
x_{m-1}-1 & 0 \\
-\frac{1}{x_{m-1}} & x_{m-1}
\end{array}\right), \quad X_{m-1}^{\prime} \sim\left(\begin{array}{cc}
1-\frac{1}{x_{m-1}} & 1 \\
0 & 1
\end{array}\right), \\
X_{m} \sim\left(\begin{array}{cc}
1 & 0 \\
\frac{1}{x_{m-1}}-1 & 1
\end{array}\right), \quad X_{m}^{\prime} \sim\left(\begin{array}{ll}
1 & 0 \\
0 & 1
\end{array}\right),
\end{gathered}
$$

noting that $x_{m}=0$. By calculating $D_{1}$ at the bottom of an open two-bridge knot similarly as above, the corresponding part of $D_{1}$ is presented by

$$
\left(\begin{array}{ccc|cc}
\ddots & \ddots & \ddots & & \\
\hline 1 & 0 & 0 & 0 & \mathcal{Z}_{m}^{-1} \\
0 & 1 & 0 & \mathcal{X}_{m-1} \mathcal{X}_{m-1}^{\prime}-1 & -\mathcal{X}_{m} \\
0 & 0 & 1 & -\mathcal{X}_{m-1}^{\prime} & 0
\end{array}\right)
$$

with respect to the basis $\left(e_{W_{m-1}}, e_{X_{m-1}}, e_{Z_{m-1}}, e_{X_{m}}, e_{Z_{m}}\right)$. We multiply the matrix (32) by

$$
\operatorname{diag}\left(\operatorname{ad}\left(\begin{array}{ll}
1 & 1 \\
1 & 0
\end{array}\right)^{-1} \operatorname{ad}\left(\begin{array}{cc}
x_{m-1} & 1 \\
1 & 0
\end{array}\right)^{-1} \operatorname{ad}\left(\begin{array}{ll}
1 & 1 \\
1 & 0
\end{array}\right)^{-1}\right)
$$

from the left, and by

$$
\operatorname{diag}\left(\operatorname{ad}\left(\begin{array}{ll}
1 & 1 \\
1 & 0
\end{array}\right) \text { ad }\left(\begin{array}{cc}
x_{m-1} & 1 \\
1 & 0
\end{array}\right) \text { ad }\left(\begin{array}{ll}
1 & 1 \\
1 & 0
\end{array}\right) \text { ad }\left(\begin{array}{ll}
0 & 1 \\
1 & 0
\end{array}\right) \text { ad }\left(\begin{array}{ll}
1 & 1 \\
1 & 0
\end{array}\right)\right)
$$

from the right. Then the entries of the resulting matrix are presented by

$$
\operatorname{ad}\left(\begin{array}{ll}
1 & 1 \\
1 & 0
\end{array}\right)^{-1} \cdot \mathcal{Z}_{m}^{-1} \cdot \operatorname{ad}\left(\begin{array}{ll}
1 & 1 \\
1 & 0
\end{array}\right)=\left(\begin{array}{cc|c}
1 & -2 & -1 \\
0 & 1 & 1 \\
\hline 0 & 0 & 1
\end{array}\right),
$$




$$
\begin{aligned}
\operatorname{ad}\left(\begin{array}{cc}
x_{m-1} & 1 \\
1 & 0
\end{array}\right)^{-1} \cdot\left(\mathcal{X}_{m-1} \mathcal{X}_{m-1}^{\prime}-1\right) \cdot \operatorname{ad}\left(\begin{array}{ll}
0 & 1 \\
1 & 0
\end{array}\right) & =\left(\begin{array}{cc|c}
\frac{2 x_{m-1}+1}{x_{m-1}^{2}} & * & * \\
1 & * & * \\
0 & 0 & 0
\end{array}\right) \\
\operatorname{ad}\left(\begin{array}{cc|c|c}
x_{m-1} & 1 \\
1 & 0
\end{array}\right)^{-1} \cdot\left(-\mathcal{X}_{m}\right) \cdot \operatorname{ad}\left(\begin{array}{ll}
1 & 1 \\
1 & 0
\end{array}\right) & =\left(\begin{array}{ccc}
-\frac{1}{x_{m-1}^{2}} & * & * \\
0 & -1 & * \\
\hline 0 & 0 & -x_{m-1}^{2}
\end{array}\right), \\
\operatorname{ad}\left(\begin{array}{ll}
1 & 1 \\
1 & 0
\end{array}\right)^{-1} \cdot\left(-\mathcal{X}_{m-1}^{\prime}\right) \cdot \operatorname{ad}\left(\begin{array}{ll}
0 & 1 \\
1 & 0
\end{array}\right) & =\left(\begin{array}{cc|c}
\frac{x_{m-1}}{1-x_{m-1}} & 0 & 0 \\
0 & -1 & 0 \\
\hline 0 & 0 & \frac{1-x_{m-1}}{x_{m-1}}
\end{array}\right) .
\end{aligned}
$$

Hence, the restriction of the matrix (32) to the subcomplex $\widehat{C}^{*}$ is presented by

$$
\left(\begin{array}{cc|cc|cc|cc|cc}
\ddots & \ddots & \ddots & \ddots & \ddots & \ddots & & & & \\
\hline 1 & 0 & 0 & 0 & 0 & 0 & 0 & 0 & 1 & -2 \\
0 & 1 & 0 & 0 & 0 & 0 & 0 & 0 & 0 & 1 \\
\hline 0 & 0 & 1 & 0 & 0 & 0 & \frac{2 x_{m-1}+1}{x_{m-1}^{2}} & * & * & * \\
0 & 0 & 0 & 1 & 0 & 0 & 1 & * & * & * \\
\hline 0 & 0 & 0 & 0 & 1 & 0 & \frac{x_{m-1}}{1-x_{m-1}} & 0 & 0 & 0 \\
0 & 0 & 0 & 0 & 0 & 1 & 0 & -1 & 0 & 0
\end{array}\right) .
$$

When we calculate

$$
\operatorname{det}\left(\widehat{E}_{2} \widehat{D}_{1} \widehat{E}_{1}\right)
$$

we remove the rightmost three columns of $\widehat{D}_{1}$ from the definition of $\widehat{E}_{1}$. We remove the rightmost three columns from the above matrix, and insert each of $e_{1}, e_{2}, e_{3}$ into the first row. Then putting

$$
\alpha_{m-1}=\frac{x_{m-1}}{x_{m-1}-1}
$$

their determinants are equal to

$$
\frac{x_{m-1}}{x_{m-1}-1}, \quad-\frac{x_{m-1}}{x_{m-1}-1}, \quad 2 \cdot \frac{x_{m-1}}{x_{m-1}-1}
$$

respectively. Hence, we define $\Phi_{2}$ of the bottom part of an open two-bridge knot by (23). 
Calculation of (24) We calculate the contribution from the other bottom part of an open two-bridge knot to the required value, as follows.
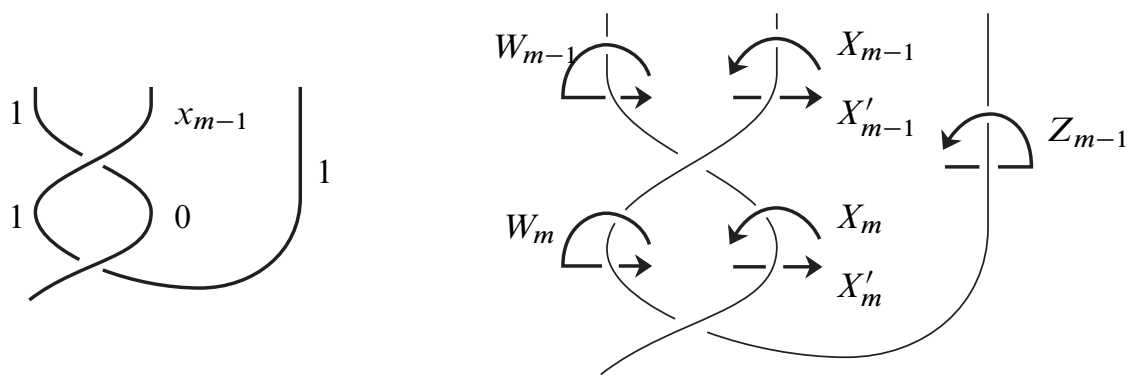

As explained in Section 3.1, we have that

$$
\begin{gathered}
X_{m-1} \sim\left(\begin{array}{cc}
x_{m-1} & 0 \\
\frac{1}{x_{m-1}} & x_{m-1}-1
\end{array}\right), \quad X_{m-1}^{\prime} \sim\left(\begin{array}{cc}
-1 & 1 \\
0 & \frac{1}{x_{m-1}}-1
\end{array}\right), \\
X_{m} \sim\left(\begin{array}{cc}
1 & 0 \\
1-\frac{1}{x_{m-1}} & 1
\end{array}\right), \quad X_{m}^{\prime} \sim\left(\begin{array}{ll}
1 & 0 \\
0 & 1
\end{array}\right),
\end{gathered}
$$

noting that $x_{m}=0$. By calculating $D_{1}$ at the bottom of an open two-bridge knot similarly as above, the corresponding part of $D_{1}$ is presented by

$$
\left(\begin{array}{ccc|cc}
\ddots & \ddots & \ddots & & \\
\hline 1 & 0 & 0 & -\mathcal{X}_{m-1}^{\prime-1} & 0 \\
0 & 1 & 0 & \mathcal{X}_{m-1}^{\prime-1} \mathcal{X}_{m}^{-1}\left(1-\mathcal{W}_{m}\right) & -\mathcal{X}_{m-1}^{\prime-1} \mathcal{X}_{m}^{-1} \\
0 & 0 & 1 & 0 & \mathcal{Z}_{m-1}
\end{array}\right)
$$

with respect to the basis $\left(e_{W_{m-1}}, e_{X_{m-1}}, e_{Z_{m-1}}, e_{X_{m}}, e_{W_{m}}\right)$. We multiply the matrix (33) by

$$
\operatorname{diag}\left(\operatorname{ad}\left(\begin{array}{ll}
1 & 1 \\
1 & 0
\end{array}\right)^{-1} \operatorname{ad}\left(\begin{array}{cc}
x_{m-1} & 1 \\
1 & 0
\end{array}\right)^{-1} \operatorname{ad}\left(\begin{array}{ll}
1 & 1 \\
1 & 0
\end{array}\right)^{-1}\right)
$$

from the left, and by

$$
\operatorname{diag}\left(\operatorname{ad}\left(\begin{array}{ll}
1 & 1 \\
1 & 0
\end{array}\right) \operatorname{ad}\left(\begin{array}{cc}
x_{m-1} & 1 \\
1 & 0
\end{array}\right) \text { ad }\left(\begin{array}{ll}
1 & 1 \\
1 & 0
\end{array}\right) \text { ad }\left(\begin{array}{ll}
0 & 1 \\
1 & 0
\end{array}\right) \text { ad }\left(\begin{array}{ll}
1 & 1 \\
1 & 0
\end{array}\right)\right)
$$

from the right. Then the entries of the resulting matrix are presented by

$$
\operatorname{ad}\left(\begin{array}{ll}
1 & 1 \\
1 & 0
\end{array}\right)^{-1} \cdot\left(-\mathcal{X}_{m-1}^{\prime}{ }^{-1}\right) \cdot \operatorname{ad}\left(\begin{array}{ll}
0 & 1 \\
1 & 0
\end{array}\right)=\left(\begin{array}{cc|c}
\frac{x_{m-1}}{1-x_{m-1}} & 0 & 0 \\
0 & -1 & 0 \\
\hline 0 & 0 & \frac{1-x_{m-1}}{x_{m-1}}
\end{array}\right),
$$




$$
\begin{aligned}
& \operatorname{ad}\left(\begin{array}{cc}
x_{m-1} & 1 \\
1 & 0
\end{array}\right)^{-1} \cdot\left(-\mathcal{X}_{m-1}^{\prime}{ }^{-1} \mathcal{X}_{m}^{-1}\right) \cdot \operatorname{ad}\left(\begin{array}{ll}
1 & 1 \\
1 & 0
\end{array}\right)=\left(\begin{array}{cc|c}
*-\frac{2}{x_{m-1}} & * \\
0 & -1 & * \\
\hline 0 & 0 & x_{m-1}\left(1-x_{m-1}\right)
\end{array}\right), \\
& \operatorname{ad}\left(\begin{array}{cc}
x_{m-1} & 1 \\
1 & 0
\end{array}\right)^{-1} \cdot \mathcal{X}_{m-1}^{\prime}{ }^{-1} \mathcal{X}_{m}^{-1}\left(1-\mathcal{W}_{m}\right) \cdot \operatorname{ad}\left(\begin{array}{ll}
0 & 1 \\
1 & 0
\end{array}\right) \\
& =\left(\begin{array}{cc|c}
\frac{2 x_{m-1}-1}{x_{m-1}\left(x_{m-1}-1\right)} & \frac{4}{x_{m-1}} & * \\
1 & 2 & -1 \\
\hline 0 & 0 & 0
\end{array}\right), \\
& \operatorname{ad}\left(\begin{array}{ll}
1 & 1 \\
1 & 0
\end{array}\right)^{-1} \cdot \mathcal{Z}_{m-1} \cdot \operatorname{ad}\left(\begin{array}{ll}
1 & 1 \\
1 & 0
\end{array}\right)=\left(\begin{array}{cc|c}
1 & 2 & -1 \\
0 & 1 & -1 \\
\hline 0 & 0 & 1
\end{array}\right)
\end{aligned}
$$

Hence, the restriction of the matrix (33) to the subcomplex $\hat{C}^{*}$ is presented by

$$
\left(\begin{array}{cc|cc|cc|cc|cc}
\ddots & \ddots & \ddots & \ddots & \ddots & \ddots & & & & \\
\hline 1 & 0 & 0 & 0 & 0 & 0 & \frac{x_{m-1}}{1-x_{m-1}} & 0 & 0 & 0 \\
0 & 1 & 0 & 0 & 0 & 0 & 0 & -1 & 0 & 0 \\
\hline 0 & 0 & 1 & 0 & 0 & 0 & \frac{2 x_{m-1}-1}{x_{m-1}\left(x_{m-1}-1\right)} & * & * & * \\
0 & 0 & 0 & 1 & 0 & 0 & 1 & * & * & * \\
\hline 0 & 0 & 0 & 0 & 1 & 0 & 0 & 0 & 1 & 2 \\
0 & 0 & 0 & 0 & 0 & 1 & 0 & 0 & 0 & 1
\end{array}\right) .
$$

Similarly as the above case, we remove the rightmost three columns from the above matrix, and insert each of $e_{1}, e_{2}, e_{3}$ into the first row. Then setting $\alpha_{m-1}=1-1 / x_{m-1}$, their determinants are equal to $-2,1,-1$, respectively. Hence, we define $\Phi_{2}$ of this bottom part of an open two-bridge knot by (24).

Therefore, by defining $\Phi_{2}$ as in the statement of the lemma, the required value is calculated as mentioned in the lemma. This completes the proof of the lemma.

\subsection{Calculation of $\operatorname{det}\left(\check{D}_{1} \check{E}_{1}\right)$}

In this section, we calculate $\operatorname{det}\left(\breve{D}_{1} \breve{E}_{1}\right)$ for any two-bridge knot. We calculate it by using a "representation" of parameterized 3-braids as we show in Lemma 3.3 below. The aim of this section is to prove this lemma.

As mentioned in Section 3.3, any open two-bridge knot can be obtained by gluing copies of elementary diagrams. 
Lemma 3.3 Let $D$ be a diagram of any open two-bridge knot, obtained by gluing

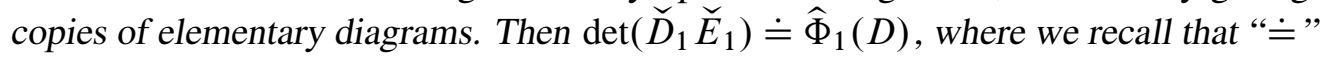
means that the left-hand side is equal to the right-hand side up to \pm 1 , and we define $\widehat{\Phi}_{1}(D)$ to be the product of $\widehat{\Phi}_{1}$ of elementary diagrams whose values are:

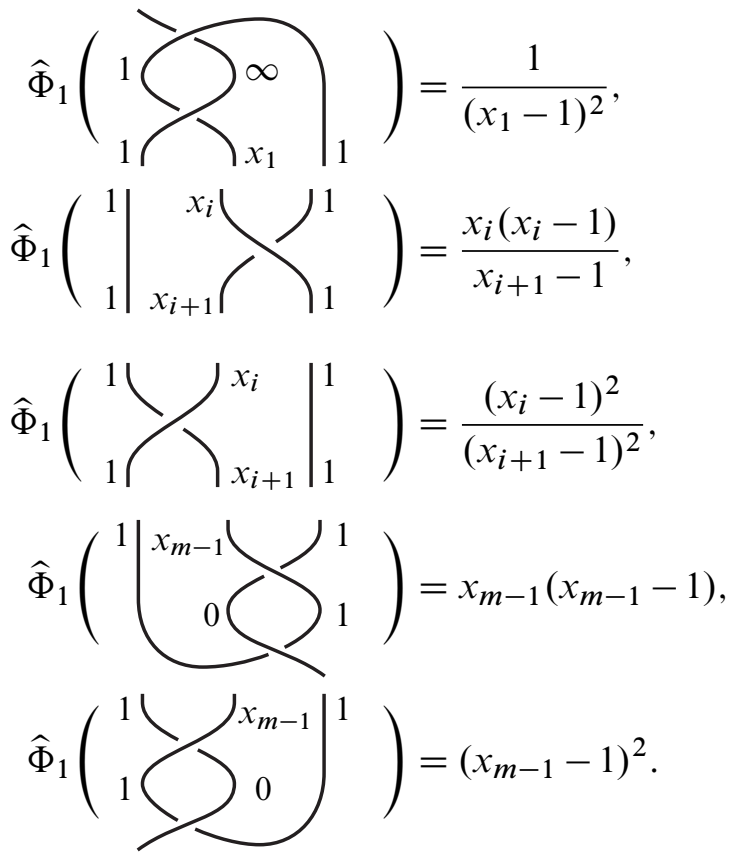

Proof Similarly as in Section 3.4, we can see that at the top part of an open two-bridge knot diagram, $\breve{D}_{1}$ is presented by

$$
\left(\begin{array}{cc|ccc}
1 & 0 & 0 & 0 & 1 \\
1 & 0 & 0 & \frac{1}{x_{1}-1} & 0 \\
0 & 1 & \frac{1}{1-x_{1}} & 0 & 0 \\
\hline 0 & 0 & \ddots & \ddots & \ddots
\end{array}\right)
$$

with respect to the basis $e_{W_{0}}, e_{X_{0}}, e_{W_{1}}, e_{X_{1}}, e_{Z_{1}}$. Further, at the part of $\sigma_{1}, \check{D}_{1}$ is presented by

$$
\left(\begin{array}{ccc|ccc}
\ddots & \ddots & \ddots & & & \\
\hline 1 & 0 & 0 & 0 & \frac{x_{i}-1}{x_{i}\left(x_{i}+1\right)} & 0 \\
0 & 1 & 0 & \frac{x_{i}\left(x_{i}-1\right)}{x_{i+1}-1} & 0 & 0 \\
0 & 0 & 1 & 0 & 0 & -1 \\
\hline & & \ddots & \ddots & \ddots
\end{array}\right)
$$


with respect to the basis $e_{W_{i}}, e_{X_{i}}, e_{Z_{i}}, e_{W_{i+1}}, e_{X_{i+1}}, e_{Z_{i+1}}$. At the part of $\sigma_{2}^{-1}, \check{D}_{1}$ is presented by

$$
\left(\begin{array}{ccc|ccc}
\ddots & \ddots & \ddots & & & \\
\hline 1 & 0 & 0 & -1 & 0 & 0 \\
0 & 1 & 0 & 0 & 0 & \frac{x_{i}\left(x_{i}-x_{i+1}\right)}{x_{i+1}-1} \\
0 & 0 & 1 & 0 & \frac{x_{i}-1}{x_{i+1}-x_{i}} & 0 \\
\hline & & & \ddots & \ddots & \ddots
\end{array}\right)
$$

with respect to the basis $e_{W_{i}}, e_{X_{i}}, e_{Z_{i}}, e_{W_{i+1}}, e_{X_{i+1}}, e_{Z_{i+1}}$. At the bottom parts of an open two-bridge knot diagram, $\bar{D}_{1}$ are presented by

$$
\begin{gathered}
\left(\begin{array}{ccc|cc}
\ddots & \ddots & \ddots & & \\
\hline 1 & 0 & 0 & 0 & 1 \\
0 & 1 & 0 & 0 & -x_{m-1}^{2} \\
0 & 0 & 1 & \frac{1-x_{m-1}}{x_{m-1}} & 0
\end{array}\right), \\
\left(\begin{array}{ccc|cc}
\ddots & \ddots & \ddots & & 0 \\
\hline 1 & 0 & 0 & \frac{1-x_{m-1}}{x_{m-1}} & 0 \\
0 & 1 & 0 & 0 & x_{m-1}\left(1-x_{m-1}\right) \\
0 & 0 & 1 & 0 & 1
\end{array}\right),
\end{gathered}
$$

respectively, with respect to the bases $e_{W_{m-1}}, e_{X_{m-1}}, e_{Z_{m-1}}, e_{X_{m}}, e_{Z_{m}}$ and $e_{W_{m-1}}$, $e_{X_{m-1}}, e_{Z_{m-1}}, e_{X_{m}}, e_{W_{m}}$. The matrix of $\check{D}_{1}$ is a union of copies of the above mentioned matrices.

From the definition of $\check{E}_{1}$, the matrix of $\check{D}_{1} \check{E}_{1}$ is the matrix obtained from $\check{D}_{1}$ by removing the second column. Its determinant is equal to the product of some entries of $\check{D}_{1}$, since most of the entries of $\check{D}_{1}$ are equal to 0 . The choice of entries which contribute to the determinant depends on the orientations of strands; more concretely, we choose the following values depending on the orientations of strands, whose product presents the value of the required determinant:
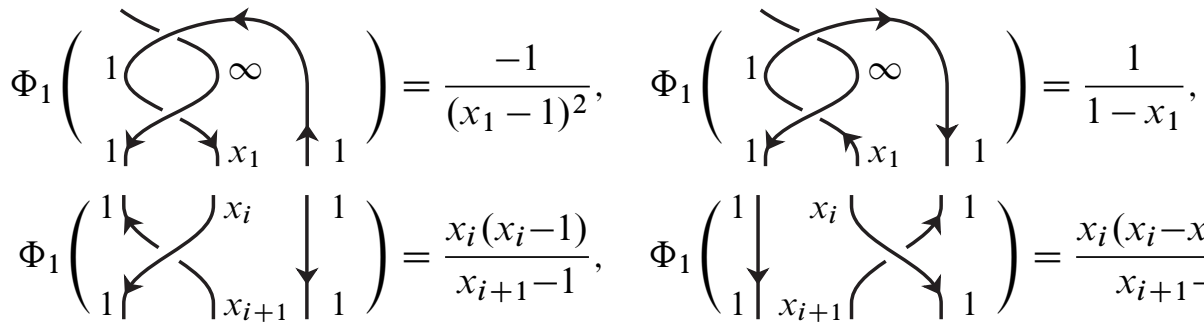

$$
\Phi_{1}\left(\Psi_{1}^{1} \begin{array}{c}
x_{i} \\
x_{i+1}
\end{array} \searrow_{1}^{1} \lambda_{1}\right)=\frac{x_{i}\left(x_{i}-x_{i+1}\right)}{x_{i+1}-1} \text {, }
$$




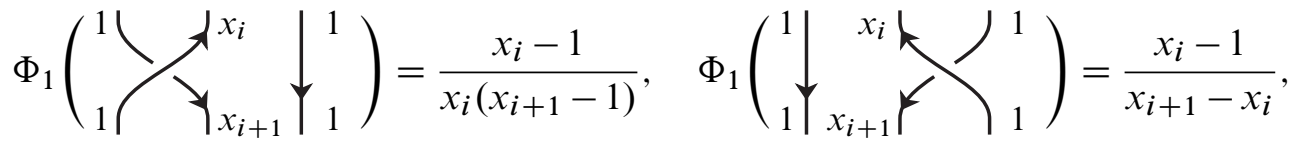

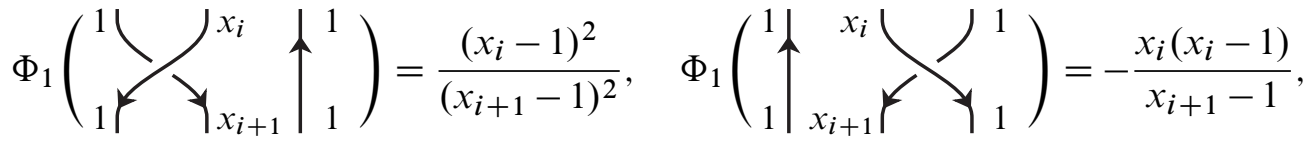

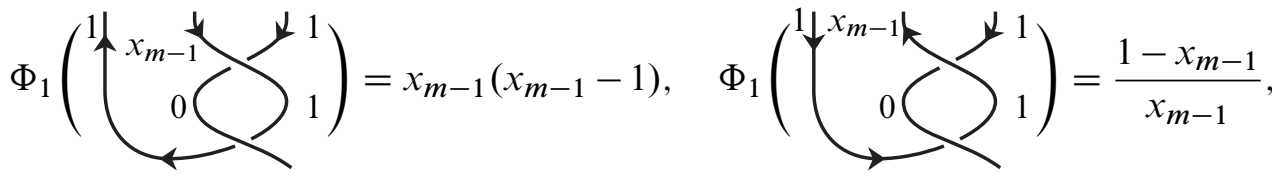
$\left.\Phi_{1}(\underbrace{1}_{1} \underbrace{x_{m-1} \uparrow^{1}}_{0})=\left(x_{m-1}-1\right)^{2}, \quad \Phi_{1}(\sum_{0}^{1} \underbrace{x_{m-1}}\}^{1}\right)=\frac{1-x_{m-1}}{x_{m-1}}$.

For a diagram $D$ of any open two-bridge knot, by decomposing $D$ into a union of elementary diagrams, we define $\Phi_{1}(D)$ to be the composition of $\Phi_{1}$ of such elementary diagrams, whose values are given above. Then by the above arguments, we have that

$$
\operatorname{det}\left(\check{D}_{1} \check{E}_{1}\right) \doteq \Phi_{1}(D) .
$$

For an elementary tangle diagram $T$, we can obtain $\widehat{\Phi}_{1}(T)$ of the lemma from $\Phi_{1}(T)$ (ignoring the difference of sign) by multiplying by

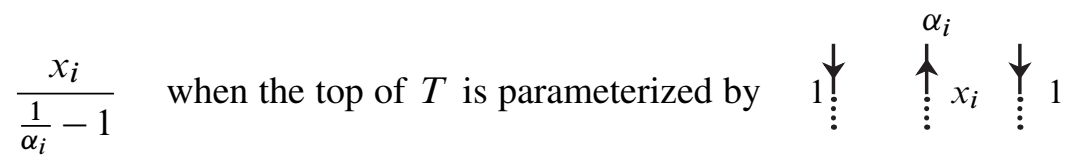

and multiplying by

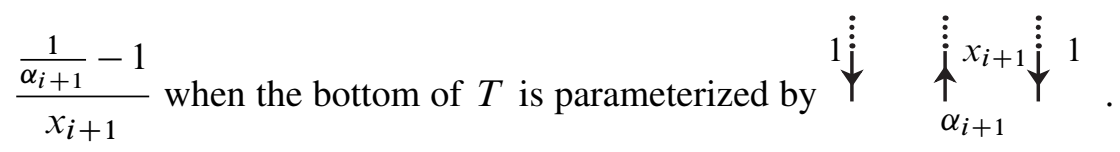

In particular, we can verify that $\widehat{\Phi}_{1}(T)$ does not depend on the orientation of $T$ ignoring the difference of sign. Hence, we obtain the required formula of the lemma.

\subsection{Calculation of the twisted Reidemeister torsion for any two-bridge knot}

In this section, we calculate the twisted Reidemeister torsion $\tau(K)$ for any two-bridge knot $K$, by applying Lemmas 3.1 and 3.3 to (18). 
We define $\Phi(\cdot)$ by $\Phi(\cdot)=\widehat{\Phi}_{1}(\cdot) \Phi_{2}(\cdot)$. Its concrete values are given by

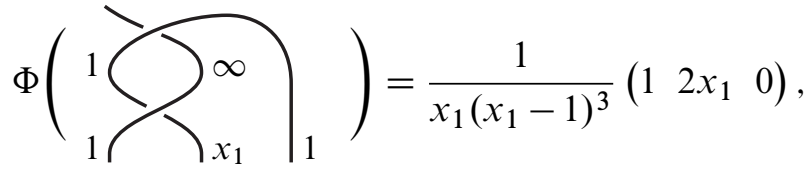

$\Phi\left(\left.\left.\int_{1}^{1}\right|_{x_{i+1}} ^{x_{i}}\right|_{1} ^{1}\right)=\frac{\left(x_{i}-1\right)^{2}}{x_{i+1}\left(x_{i+1}-1\right)^{2}}\left(\begin{array}{ccc}1 & 2 x_{i+1} & 1 \\ 0 & -x_{i+1} & -1 \\ 0 & 0 & 1\end{array}\right)$,

$\Phi\left(\begin{array}{c|c}1 & x_{i} \\ & \end{array} \sum_{x_{i+1}}^{1}{ }_{1}\right)=\frac{x_{i}^{2}}{x_{i+1}}\left(\begin{array}{ccc}1 & 0 & 0 \\ -1 & -x_{i+1} & 0 \\ 1 & 2 x_{i+1} & 1\end{array}\right)$,

$\Phi\left(\begin{array}{c}1 \\ 0\end{array}\right)=x_{m-1}^{2}\left(\begin{array}{c}1 \\ -1 \\ 2\end{array}\right)$,

$\Phi\left(\sum_{1}^{1}{ }^{x_{m-1}}{ }^{1}\right)=\left(x_{m-1}-1\right)^{2}\left(\begin{array}{c}2 \\ -1 \\ 1\end{array}\right)$,

ignoring the difference of sign.

For an elementary tangle diagram $T$, we define $\widehat{\Phi}(T)$ from $\Phi(T)$ by multiplying by

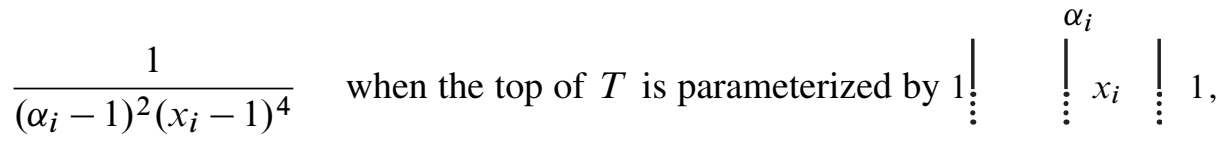

multiplying by

$\left(\alpha_{i+1}-1\right)^{2}\left(x_{i+1}-1\right)^{4}$ when the bottom of $T$ is parameterized by

and dividing the value of the bottom part by $1-\frac{1}{x_{m-1}}$. Its concrete values are given by

$$
\begin{aligned}
& \hat{\Phi}(>_{1}^{\infty} \overbrace{x_{1}})=x_{1}\left(x_{1}-1\right)\left(\begin{array}{lll}
1 & 2 x_{1} & 0
\end{array}\right) \text {, } \\
& \hat{\Phi}\left(\left.\begin{array}{l|l}
1 & x_{i} \\
1
\end{array}\right|_{x_{i+1}} ^{1}\right)=x_{i+1}\left(\begin{array}{ccc}
1 & 2 x_{i+1} & 1 \\
0 & -x_{i+1} & -1 \\
0 & 0 & 1
\end{array}\right) \text {, }
\end{aligned}
$$




$$
\begin{gathered}
\hat{\Phi}\left(\begin{array}{c}
1 \\
1
\end{array}\right)=x_{i+1}\left(\begin{array}{ccc}
1 & 0 & 0 \\
-1 & -x_{i+1} & 0 \\
1 & 2 x_{i+1} & 1
\end{array}\right), \\
\hat{\Phi}\left(\begin{array}{c}
1 \\
x_{i+1}
\end{array}\right)=\frac{x_{m-1}^{3}}{\left(x_{m-1}-1\right)^{3}}\left(\begin{array}{c}
1 \\
-1 \\
2
\end{array}\right), \\
\hat{\Phi}\left(\begin{array}{c}
x_{m-1} \\
1
\end{array}{ }^{1}\right)=\frac{x_{m-1}^{3}}{\left(x_{m-1}-1\right)^{3}}\left(\begin{array}{c}
2 \\
-1 \\
1
\end{array}\right) .
\end{gathered}
$$

By the above construction, $\Phi(D) /\left(1-\frac{1}{x_{m-1}}\right) \doteq \widehat{\Phi}(D)$ for a diagram $D$ of any open two-bridge knot.

Hence, for a diagram $D$ of any open two-bridge knot $K$, we have that

$$
\frac{2}{\tau(K)}=\widehat{\Phi}(D) \text {. }
$$

Example 3.4 We numerically calculate the twisted Reidemeister torsion for the $\overline{5_{2}}$ knot, which is the knot shown in Section 3.2. As shown in Section 3.2, the hyperbolicity equations are presented by

$$
x_{2}=x_{1}^{2}-x_{1}+1, \quad x_{2}+1-\frac{x_{2}}{x_{1}}=0 .
$$

Hence,

$$
x_{1}^{3}-2 x_{1}^{2}+3 x_{1}-1=0 .
$$

Corresponding to the holonomy representation of the hyperbolic structure of the knot complement, we choose a solution

$$
x_{1}=0.784920145 \ldots+\sqrt{-1} \cdot 1.307141278 \ldots,
$$

which gives the complex hyperbolic volume by

$$
\varsigma\left(\overline{5_{2}}\right)=\frac{1}{2 \pi \sqrt{-1}} V\left(x_{1}, x_{2}\right)=0.450109610 \ldots-\sqrt{-1} \cdot 0.4813049796 \ldots
$$

Therefore, by (34),

$$
\begin{aligned}
\frac{2}{\tau\left(\overline{5_{2}}\right)} & =x_{1}\left(x_{1}-1\right)\left(\begin{array}{lll}
1 & 2 x_{1} & 0
\end{array}\right) \cdot x_{2}\left(\begin{array}{ccc}
1 & 0 & 0 \\
-1 & -x_{2} & 0 \\
1 & 2 x_{2} & 1
\end{array}\right) \cdot \frac{x_{2}^{3}}{\left(x_{2}-1\right)^{3}}\left(\begin{array}{c}
1 \\
-1 \\
2
\end{array}\right) \\
& =-0.6323164993 \ldots+\sqrt{-1} \cdot 2.2345852998 \ldots,
\end{aligned}
$$


and hence the value of the twisted Reidemeister torsion of the $\overline{5_{2}}$ knot is given by

$$
\tau\left(\overline{5_{2}}\right)=-0.2344867659 \ldots-\sqrt{-1} \cdot 0.8286683659 \ldots
$$

We can confirm that the above value is also obtained from [26], by transforming the Reidemeister torsion associated with the longitude (of [26]) to the Reidemeister torsion associated with the meridian (the above value) as mentioned in [14].

Example 3.5 We numerically calculate the twisted Reidemeister torsion for the $\overline{6_{1}}$ knot, which is the knot shown in Section 2.2. As shown in Section 2.2, the hyperbolicity equations are presented by

$$
x_{2}=x_{1}^{2}-x_{1}+1, \quad x_{3}=x_{2}+1-\frac{x_{2}}{x_{1}}=0, \quad x_{3}+1-\frac{x_{3}}{x_{2}}=0 .
$$

Hence

$$
x_{1}^{4}-3 x_{1}^{3}+6 x_{1}^{2}-5 x_{1}+2=0 .
$$

Corresponding to the holonomy representation of the hyperbolic structure of the knot complement, we choose a solution

$$
x_{1}=0.8951233822 \ldots+\sqrt{-1} \cdot 1.5524918200 \ldots,
$$

which gives the complex hyperbolic volume by

$$
\varsigma\left(\overline{6_{1}}\right)=\frac{1}{2 \pi \sqrt{-1}} V\left(x_{1}, x_{2}, x_{3}\right)=0.5035603876 \ldots-\sqrt{-1} \cdot 1.0807800768 \ldots
$$

Therefore, by (34),

$$
\begin{aligned}
\frac{2}{\tau\left(\overline{6_{1}}\right)}= & x_{1}\left(x_{1}-1\right)\left(\begin{array}{rrr}
1 & 2 x_{1} & 0
\end{array}\right) \cdot x_{2}\left(\begin{array}{rrr}
1 & 0 & 0 \\
-1 & -x_{2} & 0 \\
1 & 2 x_{2} & 1
\end{array}\right) \\
& \times x_{3}\left(\begin{array}{ccc}
1 & 0 & 0 \\
-1 & -x_{3} & 0 \\
1 & 2 x_{3} & 1
\end{array}\right) \cdot \frac{x_{3}^{3}}{\left(x_{3}-1\right)^{3}}\left(\begin{array}{c}
1 \\
-1 \\
2
\end{array}\right) \\
& =0.9749303264 \ldots-\sqrt{-1} \cdot 3.4760907942 \ldots,
\end{aligned}
$$

and, hence, the value of the twisted Reidemeister torsion of the $\overline{6_{1}}$ knot is given by

$$
\tau\left(\overline{6_{1}}\right)=0.1496015098 \ldots+\sqrt{-1} \cdot 0.5334006103 \ldots
$$

We can confirm that the above value is also obtained from [26], by transforming the Reidemeister torsion associated with the longitude (of [26]) to the Reidemeister torsion associated with the meridian (the above value) as mentioned in [14]. 


\section{Definition and calculation of $\omega_{2}$}

In this section, we define $\omega_{2}(D)$ for an oriented parameterized open knot diagram $D$ in Section 4.1, and show that it is (formally, in general) equal (up to sign) to the square of $\omega(K)$ of the asymptotic expansion (2) of the Kashaev invariant in Section 4.2. Further, we calculate $\omega_{2}(D)$ for open two-bridge knot diagrams in Section 4.3.

\subsection{Definition of $\omega_{2}$}

In this section, we define $\omega_{2}(D)$ for an oriented parameterized open knot diagram $D$ in Definition 4.4, motivated by the square of $\omega(K)$ of the asymptotic expansion (2) of the Kashaev invariant. We show that $\omega_{2}(D)$ is invariant under the RII and RIII moves under a certain assumption on the values of hyperbolicity parameters in Proposition 4.5.

For a parameterized knot diagram $D$, we slice $D$ by horizontal lines in such a way that each region has a crossing or a critical point, we define $\Omega_{1}$ of each region as follows, and we set $\Omega_{1}(D)$ to be the product of them:

$$
\begin{aligned}
& \Omega_{1}\left(|\cdots .| \begin{array}{l}
x \\
\mid x^{\prime}
\end{array} y_{y^{\prime}}^{y}|\cdots .|\right)=\left(1-\frac{x}{x^{\prime}}\right)\left(1-\frac{y^{\prime}}{y}\right) \text {, } \\
& \left.\Omega_{1}\left(\bigcup_{1}^{1}\right\rangle_{y^{\prime}}^{y}|\cdots \cdots|\right)=1-\frac{y^{\prime}}{y}, \\
& \left.\Omega_{1}\left(\begin{array}{l|l}
\mid \cdots & x \\
& x^{\prime}
\end{array}\right\rangle_{1}^{1}\right)=1-\frac{x}{x^{\prime}}, \\
& \Omega_{1}\left(|\cdots .| \begin{array}{l}
x \\
x^{\prime}
\end{array} y_{y^{\prime}}^{y}|\cdots \ldots|\right)=\left(1-\frac{x^{\prime}}{x}\right)\left(1-\frac{y}{y^{\prime}}\right) \text {, }
\end{aligned}
$$

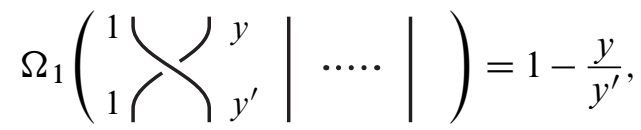

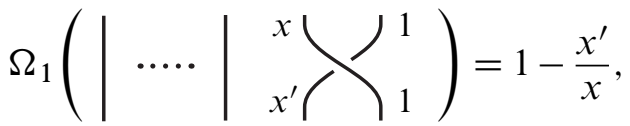

$$
\begin{aligned}
& \Omega_{1}\left(|\cdots \cdot| \bigcap_{\alpha}|\cdots \cdot|\right)=\alpha \text {, }
\end{aligned}
$$




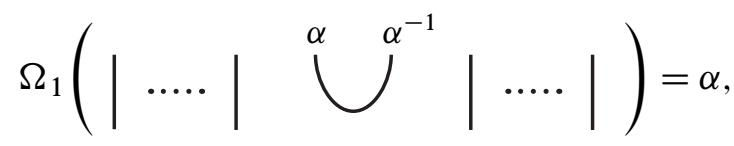

where the parameter $\alpha$ at an end of a strand is defined as in Section 3.3.

Lemma 4.1 For a parameterized knot diagram $D$, the value of $\Omega_{1}(D)$ is determined independently of the way of slicing $D$.

Proof It is sufficient to show that $\Omega_{1}(D)$ is invariant under the following moves:
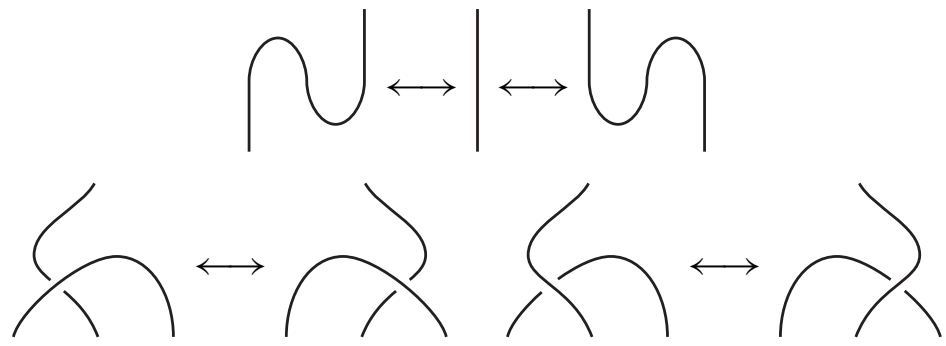

We obtain the invariance under the moves of the first line from the definition of $\Omega_{1}$.

We obtain the invariance under the moves of the second line from the definition of $\Omega_{1}$ and hyperbolicity equations among parameters.

For an oriented parameterized knot diagram $D$, define $\Omega_{2}$ of each crossing as follows, and let $\Omega_{2}(D)$ be the product of them:

$$
\Omega_{2}\left(\begin{array}{l}
x \\
x^{\prime}
\end{array} / \begin{array}{l}
y \\
y^{\prime}
\end{array}\right)=\frac{x^{\prime 2}}{x^{2}},
$$

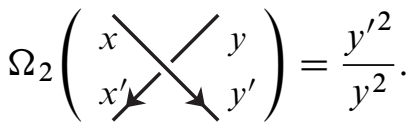

For a parameterized open knot diagram $D$, we recall that the potential function $V$ is defined as in Section 2.2, which is a function of hyperbolicity parameters $x_{i}$ 's. We also recall that a solution of hyperbolicity equations gives a critical point of $V$. We define the Hesse matrix at a critical point of $V$ by

$$
H=\left(\left(x_{i} \frac{\partial}{\partial x_{i}}\right)\left(x_{j} \frac{\partial}{\partial x_{j}}\right) V\right)_{i, j} .
$$

We note that

$$
\left(x \frac{\partial}{\partial x}\right)^{2} \operatorname{Li}_{2}\left(\frac{x}{y}\right)=\left(y \frac{\partial}{\partial y}\right)^{2} \operatorname{Li}_{2}\left(\frac{x}{y}\right)=\frac{x}{y-x}
$$


and

$$
\left(x \frac{\partial}{\partial x}\right)\left(y \frac{\partial}{\partial y}\right) \operatorname{Li}_{2}\left(\frac{x}{y}\right)=-\frac{x}{y-x},
$$

which we obtain from (11). Hence, for example, the Hesse matrix of the potential function of (10) is given by

$$
\begin{aligned}
H & =\left(\begin{array}{ccc}
\frac{x_{1}}{1-x_{1}}-\frac{1}{x_{1}-1}+\frac{x_{2}}{x_{1}-x_{2}} & -\frac{x_{2}}{x_{1}-x_{2}} & 0 \\
-\frac{x_{2}}{x_{1}-x_{2}} & \frac{x_{2}}{x_{1}-x_{2}}-\frac{x_{2}}{1-x_{2}}-\frac{1}{x_{2}-1}+\frac{x_{3}}{x_{2}-x_{3}} & -\frac{x_{3}}{x_{2}-x_{3}} \\
0 & -\frac{x_{3}}{x_{2}-x_{3}} & \frac{x_{3}}{x_{2}-x_{3}}-\frac{x_{3}}{1-x_{3}}-\frac{1}{x_{3}-1}
\end{array}\right) \\
& =\left(\begin{array}{ccc}
\frac{1+x_{1}}{1-x_{1}}+\frac{x_{2}}{x_{1}-x_{2}} & -\frac{x_{2}}{x_{1}-x_{2}} & 0 \\
-\frac{x_{2}}{x_{1}-x_{2}} & \frac{x_{2}}{x_{1}-x_{2}}+1+\frac{x_{3}}{x_{2}-x_{3}} & -\frac{x_{3}}{x_{2}-x_{3}} \\
0 & -\frac{x_{3}}{x_{2}-x_{3}} & \frac{x_{3}}{x_{2}-x_{3}}+1
\end{array}\right) .
\end{aligned}
$$

In order to define $\omega_{2}(D)$, we consider the following assumption. In the proof of Theorem 1.1 in Section 5, we consider an open two-bridge knot diagram obtained as a plat closure of a product of copies of $\sigma_{1}$ and $\sigma_{2}^{-1}$. We show later in Lemma 4.13 that such a diagram with hyperbolicity parameters satisfies this assumption.

Assumption 4.2 We assume that, for an oriented parameterized knot diagram $D$, $\Omega_{1}(D), \Omega_{2}(D)$ and det $H$ are nonzero.

In other words, from the definition of $\Omega_{1}, \Omega_{1}(D)$ is nonzero if parameters of adjacent edges at each crossing are distinct except for the case where both edges are adjacent to an unbounded region. From the definition of $\Omega_{2}, \Omega_{2}(D)$ is nonzero if parameters in the defining formula of $\Omega_{2}$ are nonzero at each crossing. Further, det $H$ is nonzero if $H$ is nondegenerate; in this case, the corresponding solution of the hyperbolicity equations is isolated.

Remark 4.3 In this remark, we explain when Assumption 4.2 is not satisfied. We have the following two cases.

Case 1: The knot diagram is "bad" For example, we consider a knot diagram $D$ having a loop of the RI move. Then it has a crossing with adjacent edges whose parameters are the same, and hence, $\Omega_{1}(D)$ is zero. In general, when a knot diagram has a "redundant" part, such a diagram might not satisfy Assumption 4.2.

Case 2: The knot is "bad" For example, we consider a satellite knot. Then the space of conjugacy classes of parabolic representations of the knot group into $\mathrm{PGL}_{2} \mathbb{C}$ might be higher-dimensional, since we can "bend" a representation along the fundamental group of an essential torus in the knot complement. Since parabolic representations are 
related to solutions of hyperbolicity equations as mentioned in Section 3.1, the space of solutions of hyperbolicity equations might be higher-dimensional. Hence, in such a case, $H$ might be degenerate and Assumption 4.2 might not be satisfied.

Definition 4.4 For an oriented parameterized open knot diagram $D$ which satisfies Assumption 4.2, we define $\omega_{2}(D)$ by

$$
\omega_{2}(D)=\frac{1}{\sqrt{-1} \Omega_{1}(D) \Omega_{2}(D) \operatorname{det} H} .
$$

As we show in Proposition 4.9 later, $\omega_{2}(D)$ (formally, in general) presents $( \pm 1)$ times the square of $\omega(K)$ of the asymptotic expansion (2) of the Kashaev invariant. Hence, we expect that this gives an invariant of an oriented parameterized open knot. The following proposition is a partial evidence of this expectation.

Proposition 4.5 For an oriented parameterized open knot diagram $D$ satisfying Assumption 4.2, $\omega_{2}(D)$ is invariant under the RII and RIII moves if the values of the hyperbolicity parameters at the moves are generic.

Here, "generic" means that both sides of the hyperbolicity equations of the knot diagrams appearing in the RII and RIII moves are always nonzero.

Proof We show the invariance under the RII move, as follows (the following proof works when $\left.x^{\prime} \neq x \neq y \neq y^{\prime}\right)$ :

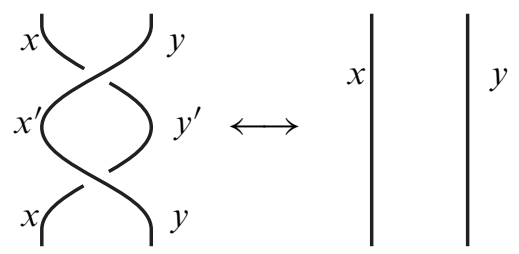

We calculate $\omega_{2}$ of the left-hand side. By definition,

$$
\Omega_{1}(\mathrm{LHS})=\left(1-\frac{x}{x^{\prime}}\right)^{2}\left(1-\frac{y^{\prime}}{y}\right)^{2}
$$

Further, we can verify by definition that

$$
\Omega_{2}(\text { LHS })=\frac{x^{\prime 2} y^{2}}{x^{2} y^{\prime 2}},
$$


independently of a choice of orientations of the strands. The Hesse matrix for the left-hand side is given by the following form:

$\left(\begin{array}{cc|cc|cc}\frac{x}{y-x}-\frac{x}{x^{\prime}-x}+a_{1} & -\frac{x}{y-x}+a_{2} & \frac{x}{x^{\prime}-x} & 0 & c_{1} & c_{2} \\ -\frac{x}{y-x}+a_{2} & \frac{x}{y-x}-\frac{y^{\prime}}{y-y^{\prime}}+a_{3} & 0 & \frac{y^{\prime}}{y-y^{\prime}} & c_{3} & c_{4} \\ \hline \frac{x}{x^{\prime}-x} & 0 & 0 & 0 & -\frac{x}{x^{\prime}-x} & 0 \\ 0 & \frac{y^{\prime}}{y-y^{\prime}} & 0 & 0 & 0 & -\frac{y^{\prime}}{y-y^{\prime}} \\ \hline c_{1} & c_{3} & -\frac{x}{x^{\prime}-x} & 0 & \frac{x}{x^{\prime}-x}-\frac{x}{y-x}+b_{1} & \frac{x}{y-x}+b_{2} \\ c_{2} & c_{4} & 0 & -\frac{y^{\prime}}{y-y^{\prime}} & \frac{x}{y-x}+b_{2} & \frac{y^{\prime}}{y-y^{\prime}}-\frac{x}{y-x}+b_{3}\end{array}\right)$

This matrix can be transformed into the following form by elementary transformations:

$$
\left(\left(\begin{array}{ll}
a_{1} & a_{2} \\
a_{2} & a_{3}
\end{array}\right)+\left(\begin{array}{ll}
b_{1} & b_{2} \\
b_{2} & b_{3}
\end{array}\right)+\left(\begin{array}{ll}
c_{1} & c_{2} \\
c_{3} & c_{4}
\end{array}\right)+\left(\begin{array}{ll}
c_{1} & c_{3} \\
c_{2} & c_{4}
\end{array}\right)\right) \oplus\left(\begin{array}{cc|cc}
0 & 0 & \frac{x}{x^{\prime}-x} & 0 \\
0 & 0 & 0 & \frac{y^{\prime}}{y-y^{\prime}} \\
\hline \frac{x}{x^{\prime}-x} & 0 & 0 & 0 \\
0 & \frac{y^{\prime}}{y-y^{\prime}} & 0 & 0
\end{array}\right)
$$

The first direct summand gives the Hesse matrix of the right-hand side. The determinant of the second direct summand is the error term, and it cancels with $\Omega_{1}$ (LHS) $\Omega_{2}$ (LHS). Hence, $\omega_{2}(D)$ is invariant under the RII move.

We show the invariance under the RIII moves, as follows (the following proof works when both sides of hyperbolicity equations appearing in the knot diagrams in the proof are always nonzero):
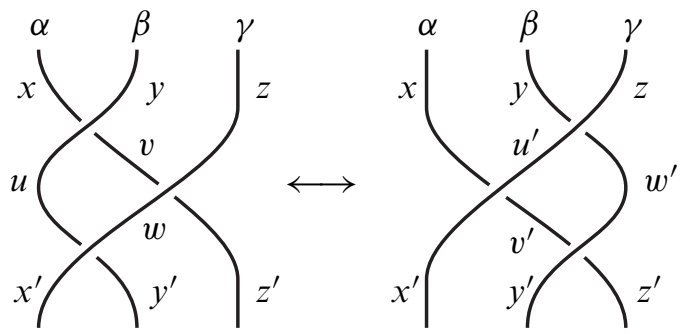

When we give values of $\alpha, \beta, \gamma, x, y, z$, the values of the other parameters are determined by

$$
\begin{aligned}
u & =\frac{x y}{\alpha x+y-\alpha y}, \quad v=\frac{x-y+\beta y}{\beta}, \\
w & =\frac{x z}{\alpha x+y-\alpha y-\beta y+\alpha \beta y+\beta z-\alpha \beta z}, \\
u^{\prime} & =\frac{y z}{\beta y+z-\beta z}, \quad w^{\prime}=\frac{y-z+\gamma z}{\gamma},
\end{aligned}
$$




$$
\begin{aligned}
v^{\prime} & =\frac{\beta x y+x z-\beta x z-\gamma x z+\beta \gamma x z-y z+\gamma y z}{\beta \gamma y}, \\
x^{\prime} & =\frac{x y z}{\alpha \beta x y+\alpha x z-\alpha \beta x z+y z-\alpha y z}, \\
y^{\prime} & =\frac{x(y-z+\gamma z)}{\gamma(\alpha x+y-\alpha y)}, \quad z^{\prime}=\frac{x-y+\beta y-\beta z+\beta \gamma z}{\beta \gamma},
\end{aligned}
$$

noting that the values of $x^{\prime}, y^{\prime}, z^{\prime}$ do not change as functions of $\alpha, \beta, \gamma, x, y, z$ under the RIII move. By definition,

$$
\begin{aligned}
& \Omega_{1}(\text { LHS })=\left(1-\frac{x}{u}\right)\left(1-\frac{v}{y}\right)\left(1-\frac{v}{w}\right)\left(1-\frac{z^{\prime}}{z}\right)\left(1-\frac{u}{x^{\prime}}\right)\left(1-\frac{y^{\prime}}{w}\right), \\
& \Omega_{1}(\mathrm{RHS})=\left(1-\frac{y}{u^{\prime}}\right)\left(1-\frac{w^{\prime}}{z}\right)\left(1-\frac{x}{x^{\prime}}\right)\left(1-\frac{v^{\prime}}{u^{\prime}}\right)\left(1-\frac{v^{\prime}}{y^{\prime}}\right)\left(1-\frac{z^{\prime}}{w^{\prime}}\right) .
\end{aligned}
$$

Further, we can verify by definition that

$$
\frac{\Omega_{2}(\mathrm{LHS})}{\Omega_{2}(\mathrm{RHS})}=\frac{y^{2} w^{2} v^{\prime 2}}{v^{2} y^{\prime 2} u^{\prime 2}},
$$

independently of a choice of orientations of the strands. The Hesse matrix of the left-hand side is given by the form

$$
\left(\begin{array}{c|c|c}
\cdots+A_{1} & B_{1} & C_{1}+\cdots \\
\hline B_{1}^{T} & D_{1} & E_{1} \\
\hline \cdots+C_{1}^{T} & E_{1}^{T} & F_{1}+\cdots
\end{array}\right),
$$

where

$$
\begin{aligned}
A_{1} & =\left(\begin{array}{ccc}
\frac{x}{y-x}-\frac{x}{u-x} & -\frac{x}{y-x} & 0 \\
-\frac{x}{y-x} & \frac{x}{y-x}-\frac{v}{y-v} & 0 \\
0 & 0 & \frac{v}{z-v}-\frac{z^{\prime}}{z-z^{\prime}}
\end{array}\right), \\
B_{1} & =\left(\begin{array}{ccc}
\frac{x}{u-x} & 0 & 0 \\
0 & \frac{v}{y-v} & 0 \\
0 & -\frac{v}{z-v} & 0
\end{array}\right), \\
C_{1} & =\left(\begin{array}{ccc}
0 & 0 & 0 \\
0 & 0 & 0 \\
0 & 0 & \frac{z^{\prime}}{z-z^{\prime}}
\end{array}\right), \\
D_{1} & =\left(\begin{array}{ccc}
\frac{v}{u-v}-\frac{x}{u-x}+\frac{u}{w-u}-\frac{u}{x^{\prime}-u} & \frac{v}{u-v}-\frac{v}{y-v}+\frac{v}{z-v}-\frac{v}{w-v} & -\frac{v}{w-u} \\
-\frac{v}{w-v} & \frac{v}{w-v} & \frac{z^{\prime}}{w-z^{\prime}}-\frac{v}{w-v}+\frac{u}{w-u}-\frac{y^{\prime}}{w-y^{\prime}}
\end{array}\right),
\end{aligned}
$$




$$
\begin{aligned}
E_{1} & =\left(\begin{array}{ccc}
\frac{u}{x^{\prime}-u} & 0 & 0 \\
0 & 0 & 0 \\
0 & \frac{y^{\prime}}{w-y^{\prime}} & -\frac{z^{\prime}}{w-z^{\prime}}
\end{array}\right), \\
F_{1} & =\left(\begin{array}{ccc}
\frac{y^{\prime}}{x^{\prime}-y^{\prime}}-\frac{u}{x^{\prime}-u} & -\frac{y^{\prime}}{x^{\prime}-y^{\prime}} & 0 \\
-\frac{y^{\prime}}{x^{\prime}-y^{\prime}} & \frac{y^{\prime}}{x^{\prime}-y^{\prime}}-\frac{y^{\prime}}{w-y^{\prime}} & 0 \\
0 & 0 & \frac{z^{\prime}}{w-z^{\prime}}-\frac{z^{\prime}}{z-z^{\prime}}
\end{array}\right) .
\end{aligned}
$$

Further, the Hesse matrix of the right-hand side is given by the form

$$
\left(\begin{array}{c|c|c}
\cdots+A_{2} & B_{2} & C_{2}+\cdots \\
\hline B_{2}^{T} & D_{2} & E_{2} \\
\hline \cdots+C_{2}^{T} & E_{2}^{T} & F_{2}+\cdots
\end{array}\right),
$$

where

$A_{2}=\left(\begin{array}{ccc}\frac{x}{u^{\prime}-x}-\frac{x}{x^{\prime}-x} & 0 & 0 \\ 0 & \frac{y}{z-y}-\frac{y}{u^{\prime}-y} & -\frac{y}{z-y} \\ 0 & -\frac{y}{z-y} & \frac{y}{z-y}-\frac{w^{\prime}}{z-w^{\prime}}\end{array}\right)$,

$B_{2}=\left(\begin{array}{ccc}-\frac{x}{u^{\prime}-x} & 0 & 0 \\ \frac{y}{u^{\prime}-y} & 0 & 0 \\ 0 & 0 & \frac{w^{\prime}}{z-w^{\prime}}\end{array}\right)$,

$C_{2}=\left(\begin{array}{ccc}\frac{x}{x^{\prime}-x} & 0 & 0 \\ 0 & 0 & 0 \\ 0 & 0 & 0\end{array}\right)$

$D_{2}$

$=\left(\begin{array}{ccc}\frac{w^{\prime}}{u^{\prime}-w^{\prime}}-\frac{y}{u^{\prime}-y}+\frac{x}{u^{\prime}-x}-\frac{v^{\prime}}{u^{\prime}-v^{\prime}} & \frac{v^{\prime}}{u^{\prime}-v^{\prime}} & -\frac{w^{\prime}}{u^{\prime}-w^{\prime}} \\ \frac{v^{\prime}}{u^{\prime}-v^{\prime}} & \frac{v^{\prime}}{x^{\prime}-v^{\prime}}-\frac{v^{\prime}-v^{\prime}}{u^{\prime}}+\frac{v^{\prime}}{w^{\prime}-v^{\prime}}-\frac{v^{\prime}}{y^{\prime}-v^{\prime}} & -\frac{v^{\prime}}{w^{\prime}-v^{\prime}} \\ -\frac{w^{\prime}}{u^{\prime}-w^{\prime}} & -\frac{v^{\prime}}{w^{\prime}-v^{\prime}} & \frac{w^{\prime}}{u^{\prime}-w^{\prime}}-\frac{w^{\prime}}{z-w^{\prime}}+\frac{v^{\prime}}{w^{\prime}-v^{\prime}}-\frac{z^{\prime}}{w^{\prime}-z^{\prime}}\end{array}\right)$,

$E_{2}=\left(\begin{array}{ccc}0 & 0 & 0 \\ -\frac{v^{\prime}}{x^{\prime}-v^{\prime}} & \frac{v^{\prime}}{y^{\prime}-v^{\prime}} & 0 \\ 0 & 0 & \frac{z^{\prime}}{w^{\prime}-z^{\prime}}\end{array}\right)$,

$F_{2}=\left(\begin{array}{ccc}\frac{v^{\prime}}{x^{\prime}-v^{\prime}}-\frac{x}{x^{\prime}-x} & 0 & 0 \\ 0 & \frac{z^{\prime}}{y^{\prime}-z^{\prime}}-\frac{v^{\prime}}{y^{\prime}-v^{\prime}} & -\frac{z^{\prime}}{y^{\prime}-z^{\prime}} \\ 0 & -\frac{z^{\prime}}{y^{\prime}-z^{\prime}} & \frac{z^{\prime}}{y^{\prime}-z^{\prime}}-\frac{z^{\prime}}{w^{\prime}-z^{\prime}}\end{array}\right)$. 
These two Hesse matrices can be transformed into the following form $(i=1,2)$ by elementary transformations:

$$
\left(\begin{array}{c|c|c}
\cdots+A_{i}-B_{i} D_{i}^{-1} B_{i}^{T} & 0 & C_{i}-B_{i} D_{i}^{-1} E_{i}+\cdots \\
\hline 0 & D_{i} & 0 \\
\hline \cdots+C_{i}^{T}-E_{i}^{T} D_{i}^{-1} B_{i}^{T} & 0 & F_{i}-E_{i}^{T} D_{i}^{-i} E_{i}+\cdots
\end{array}\right)
$$

Here, the parts of "..." are the contributions from the outside of the RIII move, and they are invariant under the RIII move. Further, we can verify by direct calculation that

$$
\begin{aligned}
A_{1}-B_{1} D_{1}^{-1} B_{1}^{T} & =A_{2}-B_{2} D_{2}^{-1} B_{2}^{T}, \\
C_{1}-B_{1} D_{1}^{-1} E_{1} & =C_{2}-B_{2} D_{2}^{-1} E_{2}, \\
F_{1}-E_{1}^{T} D_{1}^{-1} E_{1} & =F_{2}-E_{2}^{T} D_{2}^{-1} E_{2} .
\end{aligned}
$$

(We can verify the first two formulas by direct calculations. Then the third formula can be obtained from the first formula by the symmetry of $\pi$ rotation of the RIII move.) Hence, the change of the determinants of the Hesse matrices is equal to the ratio of $\operatorname{det}\left(D_{1}\right)$ and $\operatorname{det}\left(D_{2}\right)$. Since we can verify by direct calculation that

$$
\operatorname{det}\left(D_{1}\right) \Omega_{1}(\mathrm{LHS}) \Omega_{2}(\mathrm{LHS})=\operatorname{det}\left(D_{2}\right) \Omega_{1}(\mathrm{RHS}) \Omega_{2}(\mathrm{RHS}),
$$

it is shown that $\omega_{2}$ is invariant under the RIII move.

Remark 4.6 As we mentioned in Remark 4.3, Definition 4.4 does not work well for some kinds of knot diagrams. It might be difficult to show that $\omega_{2}(D)$ gives a knot invariant by showing its invariance under the Reidemeister moves.

\subsection{Relation to the Kashaev invariant}

In this section, we explain that $\omega_{2}(D)$ is (formally, in general) equal (up to sign) to the square of $\omega(K)$ of the asymptotic expansion (2) of the Kashaev invariant (Proposition 4.9). We explain this in the following 3 steps. In Step 1, we explain how the calculation of the Kashaev invariant is related to the potential function. In Step 2, by using this relation, we calculate the asymptotic expansion of the Kashaev invariant for the $\overline{6_{1}}$ knot. In Step 3, we extend this case to a general case (Proposition 4.9).

Step 1 In this step, we explain how the calculation of the Kashaev invariant is related to the potential function.

We consider an oriented open knot diagram whose ends are downward oriented. We slice such a knot diagram by horizontal lines in such a way that each region has a 
crossing or a critical point. We consider a section of a knot diagram by such a horizontal line, and associate the $i^{\text {th }}$ strand on the horizontal line with the following color:

$$
k_{i-1}-\frac{n-i}{2}+\left(\begin{array}{l}
\text { the number of upward-oriented strands } \\
\text { in the right of the } i^{\text {th }} \text { strand }
\end{array}\right)
$$

where $n$ is the number of strands on the horizontal line. Here, we put $k_{0}=k_{n-1}=0$. For example, strands are colored by

$$
0 \downarrow k_{1}+\frac{1}{2} \downarrow \quad k_{2} \uparrow k_{3}-\frac{1}{2} \uparrow \quad k_{4} \downarrow k_{5}-\frac{1}{2} \uparrow \quad 0 \downarrow
$$

and

$$
-1 \uparrow k_{1}-\frac{1}{2} \downarrow k_{2}-1 \uparrow k_{3}-\frac{3}{2} \uparrow k_{4}-1 \downarrow k_{5}-\frac{1}{2} \downarrow \quad 0 \downarrow
$$

depending on the orientations of the strands. We regard $k_{i}$ as an integer parameter for even $i$, and regard $k_{i}$ as a half-integer parameter for odd $i$.

Around a maximal point, strands are colored by

$$
k_{i}+c+\frac{1}{2} \sum_{i+1}+c \quad \text { or } \quad k_{i}+c-\frac{1}{2} \sum_{i+1}+c
$$

and, in any case, $k_{i}-\frac{1}{2}=k_{i+1}$. Further, around a minimal point, strands are colored by

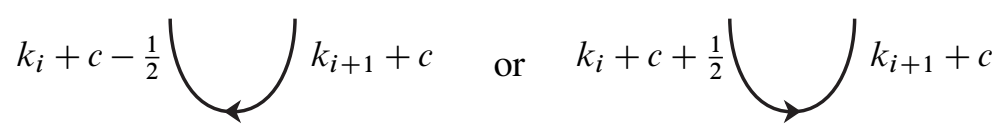

and, in any case, $k_{i}+\frac{1}{2}=k_{i+1}$. These error terms of $\frac{1}{2}$ correspond to the values of $\Omega_{1}(\cdot)^{-1 / 2}$ of critical points defined in Section 4.1 putting $q^{k_{j}}=x_{j}$.

Around a positive crossing, strands are colored by
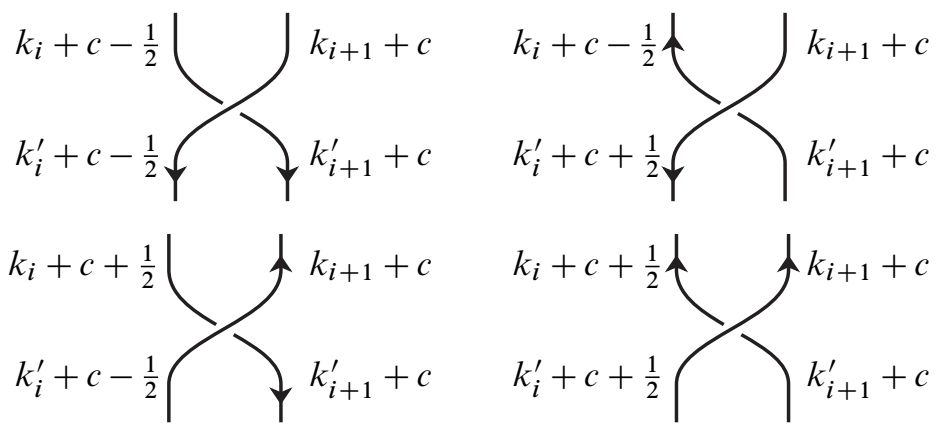
and the corresponding $R$ matrices are given by

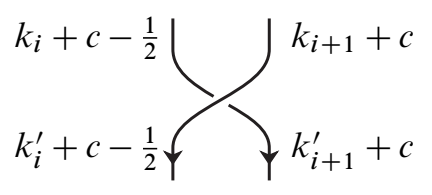

$$
R_{k_{i}^{\prime}+c-1 / 2 k_{i+1}^{\prime}+c}^{k_{i}+c-1 / 2 k_{i+1}+c} \sim \frac{N q^{k_{i}-k_{i}^{\prime}}}{(q)_{k_{i}-k_{i+1}-1 / 2}(\bar{q})_{k_{i+1}-k_{i+1}^{\prime}}(q)_{k_{i+1}^{\prime}-k_{i}^{\prime}-1 / 2}(\bar{q})_{k_{i}^{\prime}-k_{i}}},
$$

$$
\begin{aligned}
& k_{i}+c-\frac{1}{2} \\
& \bar{R}_{k_{i}+c-1 / 2 k_{i}^{\prime}+c+1 / 2}^{k_{i+1}+c k^{\prime}} \sim \frac{N q^{k_{i+1}^{\prime}}-k_{i}^{\prime}}{(\bar{q})_{k_{i+1}-k_{i+1}^{\prime}}(q)_{k_{i+1}^{\prime}-k_{i}^{\prime}-1 / 2}(\bar{q})_{k_{i}^{\prime}-k_{i}}(q)_{k_{i}-k_{i+1}-1 / 2}}, \\
& \overbrace{k_{i}^{\prime}+c-\frac{1}{2}}^{\left.k_{i}^{\prime}+c+\frac{1}{2}\right)^{k_{k+1}+c+\frac{1}{2}}} \underbrace{k_{i+1}+c}_{\substack{k_{i+1}^{\prime}+c \\
k_{i+1}^{\prime}+c}} \\
& \bar{R}_{k_{i+1}^{\prime}+c k_{i+1}+c+1}^{k_{i}^{\prime}+c+1 / 2 k_{i}+c+1 / 2} \sim \frac{N q^{k_{i}-k_{i+1}}}{(\bar{q})_{k_{i}^{\prime}-k_{i}}(q)_{k_{i}-k_{i+1}-1 / 2}(\bar{q})_{k_{i+1}-k_{i+1}^{\prime}}(q)_{k_{i+1}^{\prime}-k_{i}^{\prime}-1 / 2}},
\end{aligned}
$$

$$
\begin{aligned}
& k_{i}+c+\frac{1}{2} f k_{i+1}+c \\
& R_{k_{i+1}+c k_{i}+c+1 / 2}^{k_{i+1}^{\prime}+c k_{i}^{\prime}+c+1 / 2} \sim \frac{N q^{k_{i+1}^{\prime}-k_{i+1}}}{(q)_{k_{i+1}^{\prime}-k_{i}^{\prime}-1 / 2}(\bar{q})_{k_{i}^{\prime}-k_{i}}(q)_{k_{i}-k_{i+1}-1 / 2}(\bar{q})_{k_{i+1}-k_{i+1}^{\prime}}} .
\end{aligned}
$$

The contributions of the " $q$ " " part of the numerators of the right-hand sides to the Kashaev invariant are presented by $\Omega_{2}(\cdot)^{-1 / 2}$ defined in Section 4.1, putting $q^{k_{j}}=x_{j}$ and $q^{k_{j}^{\prime}}=x_{j}^{\prime}$. The contributions of the denominators of the right-hand sides to the Kashaev invariant are equal, and their contributions to the Kashaev invariant are presented by

$\exp \left(\cdots+\varphi\left(t_{i}-t_{i+1}\right)-\varphi\left(1-t_{i+1}+t_{i+1}^{\prime}-\frac{1}{2 N}\right)+\varphi\left(t_{i+1}^{\prime}-t_{i}^{\prime}\right)-\varphi\left(1-t_{i}^{\prime}+t_{i}-\frac{1}{2 N}\right)+\cdots\right)$, 
setting $t_{j}=k_{j} / N$ and $t_{j}^{\prime}=k_{j}^{\prime} / N$. Further, since

$$
\begin{aligned}
\varphi\left(1-t_{i+1}+t_{i+1}^{\prime}-\frac{1}{2 N}\right) & =\varphi\left(1-t_{i+1}+t_{i+1}^{\prime}\right)-\frac{1}{2 N} \varphi^{\prime}\left(1-t_{i+1}+t_{i+1}^{\prime}\right)+O\left(\frac{1}{N^{2}}\right) \\
& =\varphi\left(1-t_{i+1}+t_{i+1}^{\prime}\right)+\frac{1}{2} \log \left(1-\frac{x_{i+1}^{\prime}}{x_{i+1}}\right)+O\left(\frac{1}{N^{2}}\right), \\
\varphi\left(1-t_{i}^{\prime}+t_{i}-\frac{1}{2 N}\right) & =\varphi\left(1-t_{i}^{\prime}+t_{i}\right)+\frac{1}{2} \log \left(1-\frac{x_{i}}{x_{i}^{\prime}}\right)+O\left(\frac{1}{N^{2}}\right),
\end{aligned}
$$

the contributions from the " $\varphi(\cdots-1 / 2 N)$ " parts to the Kashaev invariant are the multiples of $\left(1-x_{i} / x_{i}^{\prime}\right)^{-1 / 2}$ and $\left(1-x_{i+1}^{\prime} / x_{i+1}\right)^{-1 / 2}$, putting $x_{j}=e^{2 \pi \sqrt{-1} t_{j}}$ and $x_{j}^{\prime}=e^{2 \pi \sqrt{-1} t_{j}^{\prime}}$, and they are presented by $\Omega_{1}(\cdot)^{-1 / 2}$ of a crossing defined in Section 4.1. By using the remaining part, we let $\breve{V}$ be the sum of the following form:

$$
\begin{array}{r}
\check{V}=\frac{1}{N}\left(\cdots+\varphi\left(t_{i}-t_{i+1}\right)-\varphi\left(1-t_{i+1}+t_{i+1}^{\prime}\right)+\varphi\left(t_{i+1}^{\prime}-t_{i}^{\prime}\right)-\varphi\left(1-t_{i}^{\prime}+t_{i}\right)+\cdots\right) \\
=\frac{1}{2 \pi \sqrt{-1}}\left(\cdots+\operatorname{Li}_{2}\left(\frac{x_{i}}{x_{i+1}}\right)-\operatorname{Li}_{2}\left(\frac{x_{i+1}^{\prime}}{x_{i+1}}\right)+\operatorname{Li}_{2}\left(\frac{x_{i+1}^{\prime}}{x_{i}^{\prime}}\right)-\operatorname{Li}_{2}\left(\frac{x_{i}}{x_{i}^{\prime}}\right)+\cdots\right) \\
+O\left(\frac{1}{N^{2}}\right),
\end{array}
$$

where we obtain the second equality by (6). By using this $\breve{V}$, we can calculate the asymptotic expansion of the Kashaev invariant, as we explain in Steps 2 and 3 below.

Step 2 In this step, we explain how we calculate the asymptotic expansion of the Kashaev invariant for the $\overline{6_{1}}$ knot, before we explain a general case in Step 3.

The $\overline{6_{1}}$ knot is the following knot, which is the mirror image of the $6_{1}$ knot.

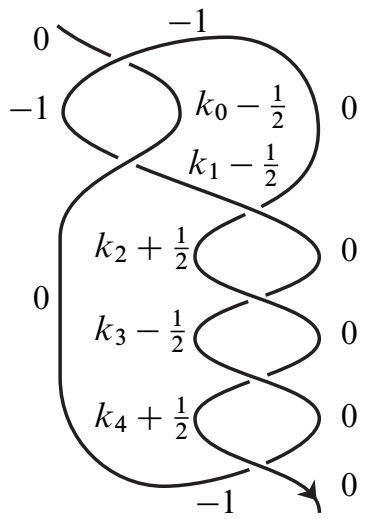

As mentioned in Step 1, the Kashaev invariant of the $\overline{6_{1}}$ knot is presented by the following form, ignoring the $q^{\text {constant }}$ terms: 
$\left\langle\overline{6_{1}}\right\rangle_{N} \sim \sum_{k_{0}, \ldots, k_{4}} \bar{R}_{k_{0}-1 / 20}^{00} \bar{R}_{-10}^{k_{0}-1 / 2 k_{1}-1 / 2} R_{k_{1}-1 / 2 k_{2}+1 / 2}^{00} R_{01}^{k_{3}+1 / 2 k_{2}+1 / 2}$

$$
\begin{aligned}
& \cdot R_{k_{3}-1 / 2 k_{4}+1 / 2}^{00} R_{01}^{0 k_{4}+1 / 2} \\
& \sim \sum_{k_{0}, \ldots, k_{4}} \frac{N}{(\bar{q})_{N-k_{0}-1 / 2}(q)_{k_{0}-1 / 2}} \times \frac{N q^{k_{1}}}{(\bar{q})_{k_{0}-k_{1}}(q)_{k_{1}-1 / 2}(q)_{N-k_{0}-1 / 2}} \\
& \times \frac{N q^{-k_{1}}}{(\bar{q})_{N-k_{2}-1 / 2}(q)_{k_{2}-k_{1}}(\bar{q})_{k_{1}-1 / 2}} \\
& \times \frac{N q^{k_{3}}}{(q)_{k_{3}-k_{2}}(\bar{q})_{k_{2}-1 / 2}(\bar{q})_{N-k_{3}-1 / 2}} \\
& \times \frac{N q^{-k_{3}}}{(\bar{q})_{N-k_{4}-1 / 2}(q)_{k_{4}-k_{3}}(\bar{q})_{k_{3}-1 / 2}} \\
& \times \frac{N}{(q)_{N-k_{4}-1 / 2}(\bar{q})_{k_{4}-1 / 2}} \\
& =\sum_{k_{1}, k_{2}, k_{3}} N^{4} \cdot q^{k_{1}} \cdot q^{-k_{1}} \cdot q^{k_{3}} \cdot q^{-k_{3}} /\left((q)_{k_{1}-1 / 2}(\bar{q})_{k_{1}-1 / 2}(q)_{k_{2}-k_{1}}\right. \\
& \left.\cdot(\bar{q})_{N-k_{2}-1 / 2}(\bar{q})_{k_{2}-1 / 2}(q)_{k_{3}-k_{2}}(\bar{q})_{N-k_{3}-1 / 2}(\bar{q})_{k_{3}-1 / 2}\right),
\end{aligned}
$$

where we obtain the last equality by (3) and (4). Hence, by (5),

$$
\left\langle\overline{6_{1}}\right\rangle_{N} \sim N^{4} \sum_{k_{1}, k_{2}, k_{3}} q^{k_{1}} \cdot q^{-k_{1}} \cdot q^{k_{3}} \cdot q^{-k_{3}} \cdot \exp \left(N \cdot \widehat{V}\left(\frac{k_{1}}{N}, \frac{k_{2}}{N}, \frac{k_{3}}{N}\right)\right),
$$

where we set

$$
\begin{aligned}
\widehat{V}\left(t_{1}, t_{2}, t_{3}\right)=\frac{1}{N}( & \varphi\left(t_{1}\right)-\varphi\left(1-t_{1}\right)+\varphi\left(t_{2}-t_{1}+\frac{1}{2 N}\right)-\varphi\left(t_{2}\right)-\varphi\left(1-t_{2}\right) \\
& \left.\quad+\varphi\left(t_{3}-t_{2}+\frac{1}{2 N}\right)-\varphi\left(t_{3}\right)-\varphi\left(1-t_{3}\right)-3 \varphi\left(\frac{1}{2 N}\right)+5 \varphi\left(1-\frac{1}{2 N}\right)\right) .
\end{aligned}
$$

Further, the " $\varphi\left(\cdots+\frac{1}{2 N}\right)$ " parts are calculated as

$$
\begin{aligned}
\varphi\left(t_{2}-t_{1}+\frac{1}{2 N}\right) & =\varphi\left(t_{2}-t_{1}\right)+\frac{\varphi^{\prime}\left(t_{2}-t_{1}\right)}{2 N}+O\left(\frac{1}{N^{2}}\right) \\
& =\varphi\left(t_{2}-t_{1}\right)-\frac{1}{2} \log \left(1-\frac{x_{2}}{x_{1}}\right)+O\left(\frac{1}{N^{2}}\right), \\
\varphi\left(t_{3}-t_{2}+\frac{1}{2 N}\right) & =\varphi\left(t_{3}-t_{2}\right)-\frac{1}{2} \log \left(1-\frac{x_{3}}{x_{2}}\right)+O\left(\frac{1}{N^{2}}\right) .
\end{aligned}
$$


Hence,

$$
\begin{aligned}
\left\langle\overline{6_{1}}\right\rangle_{N} \sim e^{\pi \sqrt{-1} / 2} \sum_{k_{1}, k_{2}, k_{3}} x_{1} \cdot x_{1}^{-1} \cdot x_{3} \cdot x_{3}^{-1}\left(\left(1-\frac{x_{2}}{x_{1}}\right)\left(1-\frac{x_{3}}{x_{2}}\right)\right)^{-1 / 2} & \cdot \exp \left(N \cdot \check{V}\left(\frac{k_{1}}{N}, \frac{k_{2}}{N}, \frac{k_{3}}{N}\right)\right),
\end{aligned}
$$

where we set

$$
\begin{aligned}
\check{V}\left(t_{1}, t_{2}, t_{3}\right)= & \frac{1}{N}\left(\varphi\left(t_{1}\right)-\varphi\left(1-t_{1}\right)+\varphi\left(t_{2}-t_{1}\right)-\varphi\left(t_{2}\right)\right. \\
& \left.\quad-\varphi\left(1-t_{2}\right)+\varphi\left(t_{3}-t_{2}\right)-\varphi\left(t_{3}\right)-\varphi\left(1-t_{3}\right)\right)+2 \cdot \frac{1}{2 \pi \sqrt{-1}} \cdot \frac{\pi^{2}}{6} \\
= & \frac{1}{2 \pi \sqrt{-1}} V\left(x_{1}, x_{2}, x_{3}\right)+O\left(\frac{1}{N^{2}}\right)
\end{aligned}
$$

putting $x_{i}=e^{2 \pi \sqrt{-1} t_{i}}$. As shown in $[17 ; 18 ; 20]$, for hyperbolic knots with up to 7 crossings, we can calculate the asymptotic expansion of the sum of the above form as

$$
\begin{aligned}
\left\langle\overline{6_{1}}\right\rangle_{N} \sim e^{\pi \sqrt{-1} / 2} N^{3} \int \Omega_{1}(D)^{-1 / 2} \Omega_{2}(D)^{-1 / 2} & \cdot \exp \left(N \cdot \check{V}\left(t_{1}, t_{2}, t_{3}\right)\right) d t_{1} d t_{2} d t_{3} \\
& \sim e^{\pi \sqrt{-1} / 2} N^{3} \Omega_{1}(D)^{-1 / 2} \Omega_{2}(D)^{-1 / 2} e^{N \varsigma\left(\overline{6_{1}}\right)} \frac{(2 \pi)^{3 / 2}}{N^{3 / 2}}(\operatorname{det}(-\check{H}))^{-1 / 2}
\end{aligned}
$$

where $D$ is a diagram of the $\overline{\sigma_{1}}$ knot mentioned above, the first approximation is an approximation of a sum by an integral which is shown by the Poisson summation formula, and the second approximation is obtained by the saddle point method at an appropriate critical point $\left(t_{1 ; c}, t_{2 ; c}, t_{3 ; c}\right)$ of $\check{V}$, letting $\breve{H}$ be the Hesse matrix at this critical point:

$$
\breve{H}=\left(\frac{\partial^{2}}{\partial t_{i} \partial t_{j}} \check{V}\right)_{i, j} .
$$

Hence, we can obtain that

$$
\left\langle\overline{6_{1}}\right\rangle_{N} \sim e^{N \varsigma\left(\overline{6_{1}}\right)} \cdot N^{3 / 2} \cdot \omega\left(\overline{6_{1}}\right),
$$

where

$$
\begin{aligned}
& \varsigma\left(\overline{\sigma_{1}}\right)=\check{V}\left(t_{1 ; c}, t_{2 ; c}, t_{3 ; c}\right), \\
& \omega\left(\overline{\sigma_{1}}\right)=e^{\pi \sqrt{-1} / 2} \Omega_{1}(D)^{-1 / 2} \Omega_{2}(D)^{-1 / 2}(2 \pi)^{3 / 2}(\operatorname{det}(-\breve{H}))^{-1 / 2} .
\end{aligned}
$$


We note that $\varsigma\left(\overline{6_{1}}\right)$ presents the complex hyperbolic volume of the complement of the $\overline{\sigma_{1}}$ knot. By the above formula, we have that

$$
\begin{aligned}
\frac{1}{\omega\left(\overline{6_{1}}\right)^{2}} & =-\Omega_{1}(D) \Omega_{2}(D) \frac{1}{(2 \pi)^{3}} \operatorname{det}(-\check{H}) \\
& \doteq \sqrt{-1} \Omega_{1}(D) \Omega_{2}(D) \operatorname{det} H,
\end{aligned}
$$

where we obtain the second equality, since $x_{i} \frac{\partial}{\partial x_{i}}=\frac{1}{2 \pi \sqrt{-1}} \frac{\partial}{\partial t_{i}}$ and hence

$$
\check{H} \sim 2 \pi \sqrt{-1} H .
$$

Therefore, from the definition of $\omega_{2}$,

$$
\omega\left(\overline{6_{1}}\right)^{2}=\omega_{2}(D) .
$$

This formula is the required formula in this step. See Example 4.11 below, for numerical verification of this formula.

Remark 4.7 In this above calculation of the asymptotic expansion of the Kashaev invariant, we have the following nontrivial two steps in (35) and (36) respectively.

(i) In (35), we approximate a sum by an integral. In fact, in a formal sense, such an approximation is a standard method (and we can guess the form of the resulting formula by formal calculation) but to be precise, this approximation is nontrivial since there is a large parameter $N$ in the exponent of the summand of the sum. As shown in $[17 ; 18 ; 20]$, we can rigorously justify this approximation for hyperbolic knots with up to 7 crossings by using the Poisson summation formula, where we must check some technical inequalities of the assumption of the Poisson summation formula; see [17; $18 ; 20]$ for details. In a general case, it is necessary to check such technical inequalities to justify the approximation of (35).

(ii) In (36), we use the multivariable saddle point method. In fact, we can guess the form of the resulting formula by formal calculation but to be precise, a nontrivial point is to make a concrete homotopy between the original domain of the integral and a new domain containing a critical point of the potential function, which satisfies the assumption of the saddle point method. For hyperbolic knots with up to 7 crossings, such a homotopy is concretely given in $[17 ; 18 ; 20]$. But in general, it is a technically complicated step to make such a homotopy concretely when we have many variables (ie when the knot diagram has many crossings).

Step 3 In this step, we extend the calculation of Step 2 to a general case. The aim of this step is to show Proposition 4.9 below. 
We recall that in the calculation of the asymptotic expansion of the Kashaev invariant, we have nontrivial two steps in (35) and (36). As mentioned in Remark 4.7, we can guess the form of the resulting formula by formal calculation in each of these steps, though, to be precise, we must check some technical inequalities of the assumptions of the Poisson summation formula and the saddle point method to justify these steps; for details see $[17 ; 18 ; 20]$. In the general case, we assume these as follows.

Assumption 4.8 We assume that, a knot diagram $D$ satisfies the above mentioned assumptions of the Poisson summation formula and the saddle point method.

Proposition 4.9 Let $K$ be an open hyperbolic knot, and let $D$ be a parameterized diagram of $K$ satisfying Assumptions 4.2 and 4.8. Then

$$
\omega(K)^{2} \doteq \omega_{2}(D) .
$$

Proof We show the proposition by extending the calculation of Step 2 to a general case, calculating the asymptotic expansion of the Kashaev invariant (by formal calculation in (35) and (36) by Assumption 4.8).

We consider an oriented open knot $K$, and consider a parameterized diagram $D$ of $K$ :

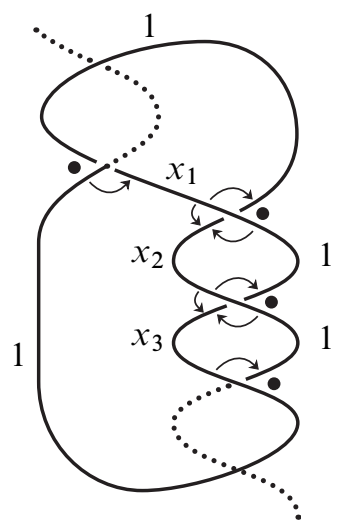

We let $n_{1}$ be the number of counterclockwise angles, let $n_{2}$ be clockwise angles, let $n$ be the number of hyperbolicity parameters, let $n_{c}$ be the number of crossings (ignoring dotted lines), and let $n_{0}$ be the number of edges parameterized by 1 (ignoring dotted lines). Since $n_{0}$ is equal to the number of angles marked by dots in the above picture, we have that

$$
n_{1}+n_{2}+n_{0}=4 n_{c}-4, \quad 2\left(n_{0}+n\right)=4 n_{c}-2 .
$$


Similarly as in the case of the $\overline{\sigma_{1}}$ knot, we can obtain that

$$
\begin{aligned}
\langle K\rangle_{N} \sim e^{\left(n_{2}-n_{1}\right) \pi \sqrt{-1} / 4} N^{n_{c}+n-\left(n_{1}+n_{2}\right) / 2} & \\
& \times \int \Omega_{1}(D)^{-1 / 2} \Omega_{2}(D)^{-1 / 2} \exp \left(N \cdot \check{V}\left(t_{1}, \ldots, t_{n}\right)\right) d t_{1} \cdots d t_{n},
\end{aligned}
$$

where

$$
\check{V}\left(t_{1}, \ldots, t_{n}\right)=\frac{1}{2 \pi \sqrt{-1}} V\left(x_{1}, \ldots, x_{n}\right)+O\left(\frac{1}{N^{2}}\right),
$$

putting $x_{i}=e^{2 \pi \sqrt{-1} t_{i}}$. We note that $n_{c}+n-\left(n_{1}+n_{2}\right) / 2=(n+3) / 2$ by (37). Hence, we obtain the following approximations (formally, in general):

$$
\begin{aligned}
& \langle K\rangle_{N} \sim e^{\left(n_{2}-n_{1}\right) \pi \sqrt{-1} / 4} N^{(n+3) / 2} \\
& \times \int \Omega_{1}(D)^{-1 / 2} \Omega_{2}(D)^{-1 / 2} \exp \left(N \cdot \breve{V}\left(t_{1}, \ldots, t_{n}\right)\right) d t_{1} \cdots d t_{n} \\
& \sim e^{\left(n_{2}-n_{1}\right) \pi \sqrt{-1} / 4} N^{(n+3) / 2} \Omega_{1}(D)^{-1 / 2} \Omega_{2}(D)^{-1 / 2} e^{N \varsigma(K)} \frac{(2 \pi)^{n / 2}}{N^{n / 2}} \\
& \cdot(\operatorname{det}(-\breve{H}))^{-1 / 2} \\
& \sim e^{\left(n_{2}-n_{1}\right) \pi \sqrt{-1} / 4} N^{3 / 2} \Omega_{1}(D)^{-1 / 2} \Omega_{2}(D)^{-1 / 2} e^{N \varsigma(K)}(2 \pi)^{n / 2} \\
& \cdot(\operatorname{det}(-\check{H}))^{-1 / 2} \text {. }
\end{aligned}
$$

Therefore

$$
\langle K\rangle_{N} \sim e^{N \varsigma(K)} \cdot N^{3 / 2} \cdot \omega(K),
$$

where

$$
\begin{aligned}
\varsigma(K) & =\breve{V}\left(t_{1 ; c}, \ldots, t_{n ; c}\right), \\
\omega(K) & =e^{\left(n_{2}-n_{1}\right) \pi \sqrt{-1} / 4} \Omega_{1}(D)^{-1 / 2} \Omega_{2}(D)^{-1 / 2}(2 \pi)^{n / 2}(\operatorname{det}(-\breve{H}))^{-1 / 2} .
\end{aligned}
$$

Further,

$$
\begin{aligned}
\frac{1}{\omega(K)^{2}} & =e^{\left(n_{1}-n_{2}\right) \pi \sqrt{-1} / 2} \Omega_{1}(D) \Omega_{2}(D) \frac{1}{(2 \pi)^{n}} \operatorname{det}(-\check{H}) \\
& =e^{\left(n_{1}-n_{2}-n\right) \pi \sqrt{-1} / 2} \Omega_{1}(D) \Omega_{2}(D) \operatorname{det} H \\
& \doteq \sqrt{-1} \Omega_{1}(D) \Omega_{2}(D) \operatorname{det} H,
\end{aligned}
$$

since $n_{1}-n_{2}-n$ is odd by (37). Hence, from the definition of $\omega_{2}$, we obtain the required formula of the proposition. 
Example 4.10 We numerically verify Proposition 4.9 for the $\overline{5_{2}}$ knot, which is the knot shown in Section 3.2. As shown in Example 3.4, we obtain the values of hyperbolicity parameters and the complex volume. Further, from the definition of $\omega_{2}$, we have that

$$
\begin{gathered}
H=\left(\begin{array}{cc}
\frac{1+x_{1}}{1-x_{1}}+\frac{x_{2}}{x_{1}-x_{2}} & -\frac{x_{2}}{x_{1}-x_{2}} \\
-\frac{x_{2}}{x_{1}-x_{2}} & \frac{x_{2}}{x_{1}-x_{2}}+1
\end{array}\right), \quad \Omega_{1}(D)=1-\frac{x_{2}}{x_{1}}, \\
\omega_{2}(D)=\frac{1}{\sqrt{-1} \Omega_{1}(D) \operatorname{det} H}=-0.4143341829 \ldots+\sqrt{-1} \cdot 0.117243382 \ldots,
\end{gathered}
$$

where $D$ is the $\overline{5_{2}}$ knot diagram shown in Section 3.2. Hence,

$$
\omega\left(\overline{5_{2}}\right)=\omega_{2}(D)^{1 / 2}=0.09019057740 \ldots+\sqrt{-1} \cdot 0.6499757866 \ldots,
$$

where we choose the sign of the square root depending the orientation of the domain of the integral of the saddle point method; for details, see [17]. Further, from the definition of the Kashaev invariant, we have that

$$
\left\langle\overline{5_{2}}\right\rangle_{N}=\sum_{0 \leq i \leq j<N} \frac{N^{3} q^{-1}}{(q)_{i}(\bar{q})_{i}(q)_{j-i}(\bar{q})_{j}(\bar{q})_{N-j-1}} ;
$$

see [17]. By calculating this sum concretely as shown in the following table, we can numerically observe that the limit of $\left\langle\overline{5_{2}}\right\rangle_{N} e^{-N \varsigma\left(\overline{5_{2}}\right)} N^{-3 / 2}$ tends to the above mentioned value of $\omega\left(\overline{5_{2}}\right)$, noting that $q \rightarrow 1$ as $N \rightarrow \infty$ :

\begin{tabular}{c|c}
$N$ & $q\left\langle\overline{5_{2}}\right\rangle_{N} e^{-N \varsigma\left(\overline{5_{2}}\right)} N^{-3 / 2}$ \\
\hline 50 & $0.09574104848 \ldots+\sqrt{-1} \cdot 0.6581517399 \ldots$ \\
100 & $0.09297541546 \ldots+\sqrt{-1} \cdot 0.6540225631 \ldots$ \\
200 & $0.09158517383 \ldots+\sqrt{-1} \cdot 0.6519891312 \ldots$
\end{tabular}

Example 4.11 We numerically verify Proposition 4.9 for the $\overline{6_{1}}$ knot, which is the knot shown in Section 2.2. As shown in Example 3.5, we obtain the values of hyperbolicity parameters and the complex volume. Further, by using the Hesse matrix shown in Section 4.1, we have that

$$
\omega_{2}(D)=\frac{1}{\sqrt{-1} \Omega_{1}(D) \operatorname{det} H}=-0.2667003051 \ldots+\sqrt{-1} \cdot 0.07480075491 \ldots,
$$

where $D$ is the $\overline{\sigma_{1}}$ knot diagram shown in Section 2.2. Hence

$$
\omega\left(\overline{6_{1}}\right)=\left(-\omega_{2}(D)\right)^{1 / 2}=-0.5213883634 \ldots+\sqrt{-1} \cdot 0.07173228265 \ldots,
$$

where we choose the sign of $\omega_{2}(D)$ depending the sign of the formula of Proposition 4.9, and choose the sign of the square root depending the orientation of the domain of the 
integral of the saddle point method; for details, see [20]. Further, from the definition of the Kashaev invariant, we have that

$$
\left\langle\overline{6_{1}}\right\rangle_{N}=\sum_{0 \leq i \leq j \leq k<N} \frac{N^{4} q^{-1}}{(q)_{i}(\bar{q})_{i}(q)_{j-i}(\bar{q})_{j}(\bar{q})_{N-j-1}(q)_{k-j}(\bar{q})_{k}(\bar{q})_{N-k-1}},
$$

as shown before in this section. By calculating this sum concretely as shown in the following table, we can numerically observe that the limit of $\left\langle\overline{6_{1}}\right\rangle_{N} e^{-N_{\varsigma}\left(\overline{6_{1}}\right)} N^{-3 / 2}$ tends to the above mentioned value of $\omega\left(\overline{6_{1}}\right)$ :

\begin{tabular}{c|c}
$N$ & $\left\langle\overline{6_{1}}\right\rangle_{N} e^{-N \varsigma\left(\overline{6_{1}}\right)} N^{-3 / 2}$ \\
\hline 50 & $-0.5121772692 \ldots+\sqrt{-1} \cdot 0.1473909514 \ldots$ \\
100 & $-0.5181425383 \ldots+\sqrt{-1} \cdot 0.1096254180 \ldots$ \\
200 & $-0.5201050838 \ldots+\sqrt{-1} \cdot 0.09068263776 \ldots$
\end{tabular}

\subsection{Calculation of $\omega_{2}$ for open two-bridge knot diagrams}

In this section, we calculate $\omega_{2}$ for open two-bridge knot diagrams. To calculate it, we introduce an operator invariant $\Psi$ and present $\omega_{2}$ in terms of $\Psi$ in Lemma 4.14 below. The aim of this section is to show this lemma.

As we explain in Section 3.3, any open two-bridge knot diagram can be obtained by gluing copies of elementary diagrams (19), ie as a plat closure of a product of copies of $\sigma_{1}$ and $\sigma_{2}^{-1}$. We consider such a diagram with hyperbolicity parameters. The hyperbolicity equation at the strand of $x_{i}$ is given by

$$
\begin{cases}\left(1-x_{i}\right)\left(1-\frac{x_{i+1}}{x_{i}}\right)=\left(1-\frac{x_{i}}{x_{i-1}}\right)\left(1-\frac{1}{x_{i}}\right) & \text { if the strand of } x_{i} \text { is between } \sigma_{1} \\ \left(1-x_{i}\right)\left(1-\frac{1}{x_{i}}\right)=\left(1-\frac{x_{i}}{x_{i-1}}\right)\left(1-\frac{x_{i+1}}{x_{i}}\right) & \text { otherwise }\end{cases}
$$

As mentioned in the definition of a parameterized diagram, we set $x_{0}=\infty$ and $x_{m}=0$, and assume that $x_{i} \in \mathbb{C}-\{0\}$ for any $i=1,2, \ldots, m-1$.

Lemma 4.12 We consider a solution ${ }^{6}$ of the system of the equations in (39) for $i=1,2, \ldots, m-1$ which satisfies that $x_{1} \neq 0,1$. Then $x_{i-1} \neq x_{i} \neq 1$ for any $i=1,2, \ldots, m$.

6 When we introduced the hyperbolicity equation in Section 2.2, we assumed that the values of both sides of the equation are nonzero. In Lemma 4.12, we consider a solution without supposing this assumption. 
Proof If $x_{2}$ were equal to 1 , it follows from (39) that

$$
\left(1-x_{1}\right)\left(1-\frac{1}{x_{1}}\right)=1-\frac{1}{x_{1}},
$$

which contradicts the assumption that $x_{1} \neq 0,1$. Hence, $x_{2} \neq 1$.

We show the lemma by induction on $i$. Assuming that the lemma holds for any $j \leq i$, we show the lemma for the case of $i+1$.

If $x_{i+1}$ was equal to 1 , it follows from (39) that

$$
\left(1-x_{i}\right)\left(1-\frac{1}{x_{i}}\right)=\left(1-\frac{x_{i}}{x_{i-1}}\right)\left(1-\frac{1}{x_{i}}\right) .
$$

Hence, $x_{i}=1$ or $x_{i-1}=1$, which contradicts the assumption of the induction. Therefore, $x_{i+1} \neq 1$.

If $x_{i}$ was equal to $x_{i+1}$, we can show by (39) that $x_{i-1}=x_{i}$ or $x_{i}=1$. This contradicts the assumption of the induction. Hence, $x_{i} \neq x_{i+1}$, as required.

For a solution satisfying Lemma 4.12, it follows from (39) that the values of hyperbolicity parameters $x_{i}$ are recursively determined from $x_{1}\left(\right.$ and $\left.x_{0}=\infty\right)$ by

$$
x_{i+1}= \begin{cases}x_{i}+1-\frac{x_{i}}{x_{i-1}} & \text { if the strand of } x_{i} \text { is between } \sigma_{1} \\ x_{i}+\frac{\left(x_{i}-1\right)^{2}}{1-x_{i} / x_{i-1}} & \text { and } \sigma_{1} \text { or } \sigma_{2}^{-1} \text { and } \sigma_{2}^{-1},\end{cases}
$$

Putting $x_{1}=x$ (and $x_{0}=\infty$ ), we can regard $x_{i}$ as a rational function of $x$. We define this rational function $f_{i}(x)$ by $f_{1}(x)=x$ (and $\left.f_{0}(x)=\infty\right)$ and

$$
f_{i+1}= \begin{cases}f_{i}+1-\frac{f_{i}}{f_{i-1}} & \text { if the strand of } x_{i} \text { is between } \sigma_{1} \\ f_{i}+\frac{\left(f_{i}-1\right)^{2}}{1-f_{i} / f_{i-1}} & \text { and } \sigma_{1} \text { or } \sigma_{2}^{-1} \text { and } \sigma_{2}^{-1},\end{cases}
$$

See Sakuma and Weeks [24] for another construction of this function, and see Appen$\operatorname{dix} \mathrm{C}$ for a construction of the numerator and the denominator of this function. The system of hyperbolicity equations is rewritten $f_{m}(x)=0$.

We choose a root $x_{1}$ of $f_{m}(x)=0$. Then by Lemma D.9, $x_{1} \neq 1$. Hence, by Lemma 4.12, the values of $x_{i}$ are recursively determined by (40), assuming ${ }^{7}$ that $x_{i} \in \mathbb{C}-\{0\}$ for each $i=1,2, \ldots, m-1$, as we did in the definition of hyperbolicity parameters.

${ }^{7}$ For many two-bridge knots, any root of $f_{m}(x)=0$ gives $x_{i} \in \mathbb{C}-\{0\}$ for any $i=1,2, \ldots, m-1$, but there exist some exceptional cases; see Remark D.12 for details. 
Lemma 4.13 The above values of $x_{i}$ satisfy Assumption 4.2.

Proof By Lemma 4.12 and the definition of hyperbolicity parameters, $x_{i-1} \neq x_{i} \neq 0,1$. Hence, from the definition of $\Omega_{1}(D)$ and $\Omega_{2}(D)$, they are nonzero.

It is sufficient to show that the Hesse matrix $H$ is nondegenerate. We recall that entries of $H$ are equal to differential coefficients of hyperbolicity equations. We consider the tangent space of the space determined by each hyperbolicity equation at the root given by the $x_{i}$. The nondegeneracy of $H$ means the transversality of the family of such tangent spaces. This condition is equivalent to the nondegeneracy of $f_{m}(x)=0$ at the root $x$. Since the equation $f_{m}(x)=0$ has no repeated root by Lemma D.8, this condition holds. Hence $H$ is nondegenerate as required.

By Lemma 4.13, we can define $\omega_{2}(D)$ of the above mentioned diagram $D$ of an open two-bridge knot.

Lemma 4.14 Let $D$ be a diagram of any open two-bridge knot, obtained by gluing copies of elementary diagrams. Then

$$
\frac{1}{\sqrt{-1} \omega_{2}(D)}=\Psi(D),
$$

where we define $\Psi(D)$ to be the composition of $\Psi$ of elementary diagrams whose values are given as follows:
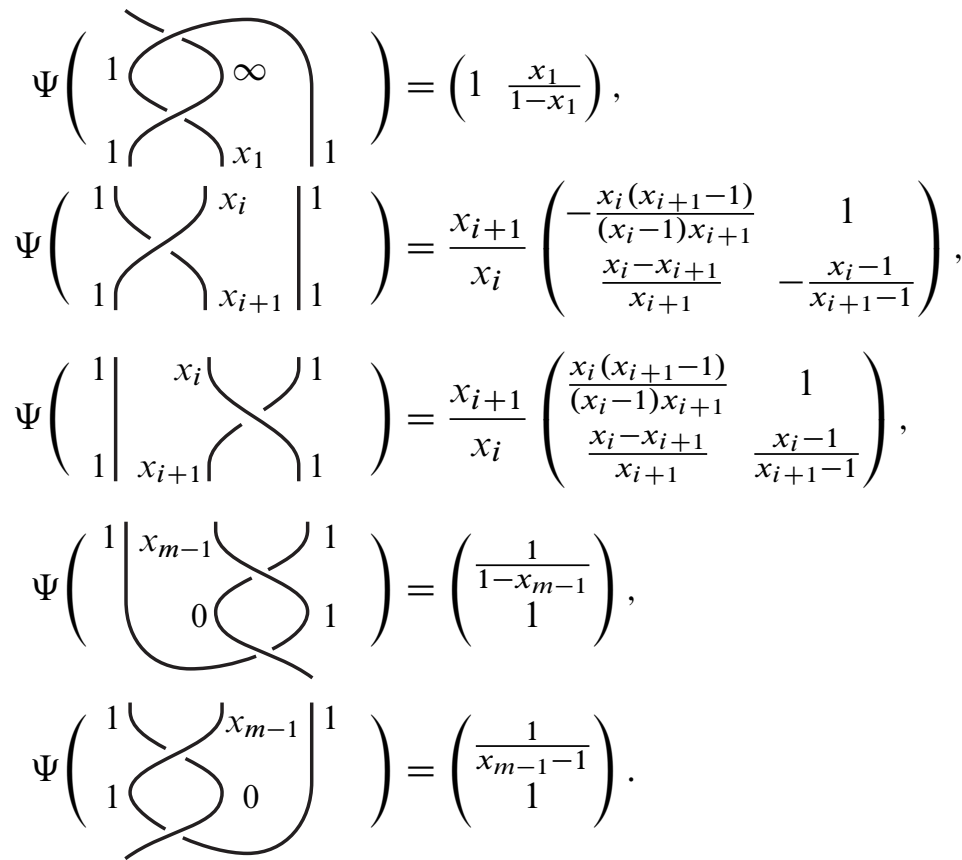
Proof By Definition 4.4, $\omega_{2}(D)$ is defined from $\Omega_{1}(D), \Omega_{2}(D)$ and the determinant of the Hesse matrix $H$. We calculate each of them in terms of elementary diagrams in the following of this proof.

From the definition of $\Omega_{1}, \Omega_{1}(D)$ is equal to the product of $\Omega_{1}$ of elementary diagrams, whose values are given as follows:

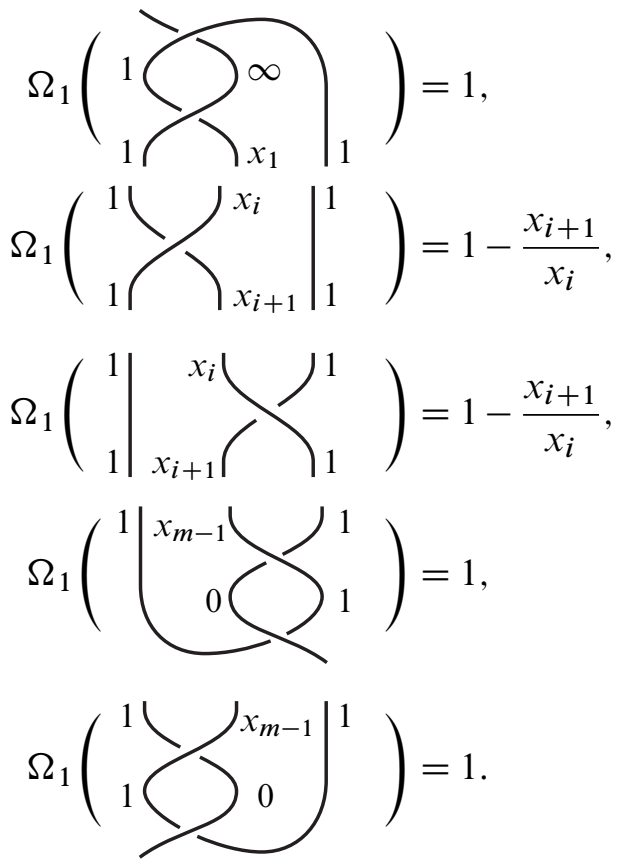

Further, from the definition of $\Omega_{2}, \Omega_{2}(D)$ is equal to the product of $\Omega_{2}$ of elementary diagrams, whose values are given as follows:

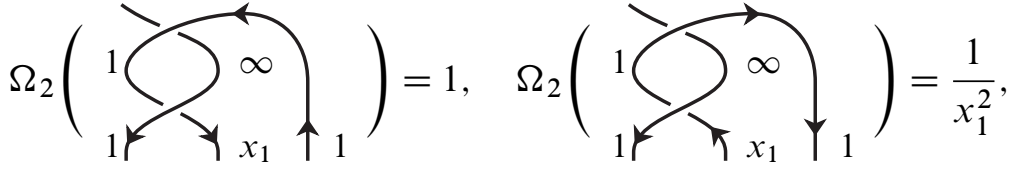

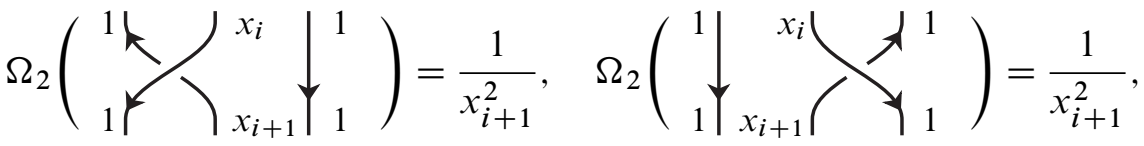

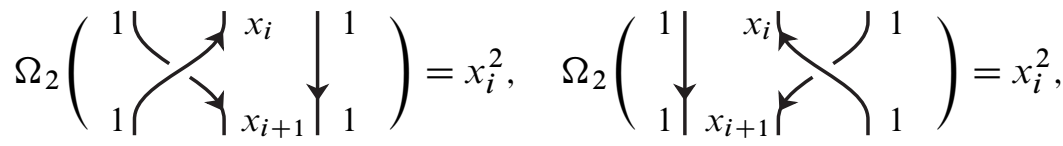




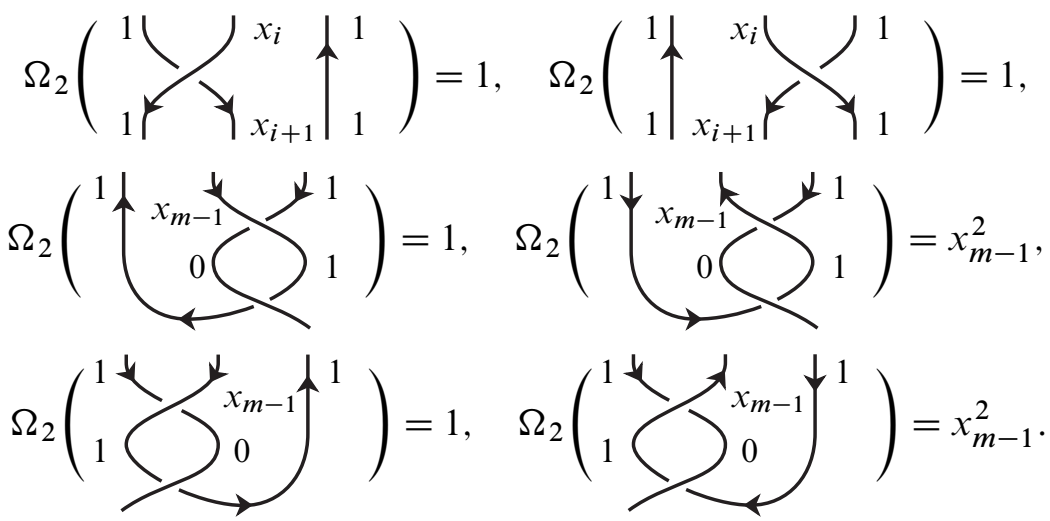

For an elementary tangle diagram $T$, we define $\widehat{\Omega}_{2}(T)$ from $\Omega_{2}(T)$ by multiplying

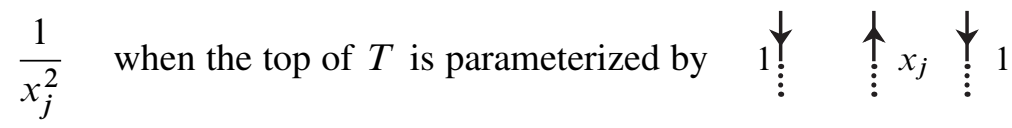
and multiplying

$x_{j+1}^{2}$ when the bottom of $T$ is parameterized by $1_{\grave{j}}^{\vdots} \quad \dot{\vdots}^{x_{j+1}} \dot{\vdots}^{1}$.

Then we can verify $\hat{\Omega}_{2}(T)=1$ for each elementary diagram $T$. Hence, $\Omega_{2}(D)=1$. We calculate the contribution of each elementary diagram to the Hesse matrix. The contribution of the diagram

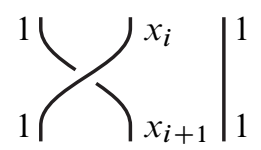

to the potential function is given by

$$
\cdots+\mathrm{Li}_{2}\left(\frac{1}{x_{i}}\right)-\mathrm{Li}_{2}\left(\frac{x_{i+1}}{x_{i}}\right)+\mathrm{Li}_{2}\left(x_{i+1}\right)+\cdots .
$$

Hence its contribution to the Hesse matrix is given by

$$
\left(\begin{array}{cccc}
\ddots & \ddots & & \\
\ddots & \cdots+\frac{1}{x_{i}-1}-\frac{x_{i+1}}{x_{i}-x_{i+1}} & \frac{x_{i+1}}{x_{i}-x_{i+1}} & \\
& \frac{x_{i+1}}{x_{i}-x_{i+1}} & -\frac{x_{i+1}}{x_{i}-x_{i+1}}+\frac{x_{i+1}}{1-x_{i+1}}+\cdots & \ddots \\
& & \ddots & \ddots
\end{array}\right)
$$


We calculate the determinant of a matrix of the above form recursively, as follows. For an indeterminant $y$, we let

$$
\operatorname{det}\left(\begin{array}{cc}
\ddots & \ddots \\
\ddots & \cdots+y
\end{array}\right)=A_{i} y+B_{i}
$$

$$
\operatorname{det}\left(\begin{array}{ccc}
\ddots & \ddots & \\
\ddots & \cdots+\frac{1}{x_{i}-1}-\frac{x_{i+1}}{x_{i}-x_{i+1}} & \frac{x_{i+1}}{x_{i}-x_{i+1}} \\
\frac{x_{i+1}}{x_{i}-x_{i+1}} & -\frac{x_{i+1}}{x_{i}-x_{i+1}}+\frac{x_{i+1}}{1-x_{i+1}}+y
\end{array}\right)=A_{i+1} y+B_{i+1} \text {. }
$$

Then we have that

$$
\begin{aligned}
& \left(\begin{array}{ll}
A_{i+1} & B_{i+1}
\end{array}\right) \\
& =\left(\begin{array}{ll}
A_{i} & B_{i}
\end{array}\right) \\
& \quad\left(\begin{array}{cc}
\frac{1}{x_{i}-1}-\frac{x_{i+1}}{x_{i}-x_{i+1}} & \left(\frac{1}{x_{i}-1}-\frac{x_{i+1}}{x_{i}-x_{i+1}}\right)\left(-\frac{x_{i+1}}{x_{i}-x_{i+1}}+\frac{x_{i+1}}{1-x_{i+1}}\right)-\left(\frac{x_{i+1}}{x_{i}-x_{i+1}}\right)^{2} \\
1 & -\frac{x_{i+1}}{x_{i}-x_{i+1}}+\frac{x_{i+1}}{1-x_{i+1}}
\end{array}\right) .
\end{aligned}
$$

Including the contribution of $\Omega_{1}$, we set

$$
\begin{aligned}
& \Psi\left(\left.\sum_{i+1}^{1}\right|_{1} ^{x_{i}} \mid \begin{array}{l}
1 \\
x_{i}
\end{array}\right) \\
&=\left(1-\frac{x_{i+1}}{x_{i}}\right) \\
& \cdot\left(\begin{array}{cc}
\frac{1}{x_{i}-1}-\frac{x_{i+1}}{x_{i}-x_{i+1}} & \left.\left(\frac{1}{x_{i}-1}-\frac{x_{i+1}}{x_{i}-x_{i+1}}\right)\left(-\frac{x_{i+1}}{x_{i}-x_{i+1}}+\frac{x_{i+1}}{1-x_{i+1}}\right)-\left(\frac{x_{i+1}}{x_{i}-x_{i+1}}\right)^{2}\right) \\
1 & -\frac{x_{i+1}}{x_{i}-x_{i+1}}+\frac{x_{i+1}}{1-x_{i+1}}
\end{array}\right), \\
&= \frac{x_{i+1}}{x_{i}}\left(\begin{array}{cc}
-\frac{x_{i}\left(x_{i+1}-1\right)}{\left(x_{i}-1\right) x_{i+1}} & 1 \\
\frac{x_{i}-x_{i+1}}{x_{i+1}} & -\frac{x_{i}-1}{x_{i+1}-1}
\end{array}\right)
\end{aligned}
$$

as in (43). Similarly, the contribution from the diagram

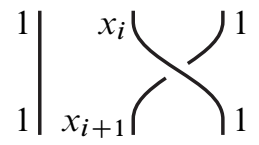


to the Hesse matrix is given by

$$
\left(\begin{array}{cccc}
\ddots & \ddots & & \\
\ddots & \cdots-\frac{1}{x_{i}-1}+\frac{x_{i+1}}{x_{i}-x_{i+1}} & -\frac{x_{i+1}}{x_{i}-x_{i+1}} & \\
& -\frac{x_{i+1}}{x_{i}-x_{i+1}} & \frac{x_{i+1}}{x_{i}-x_{i+1}}-\frac{x_{i+1}}{1-x_{i+1}}+\cdots & \ddots \\
& & \ddots & \ddots
\end{array}\right)
$$

Hence, similarly as above, we set

$$
\begin{aligned}
\Psi\left(\begin{array}{l|}
1 \\
1
\end{array}\right. & x_{i+1}{ }_{1} \\
= & \left(1-\frac{x_{i+1}}{x_{i}}\right) \\
& \cdot\left(\begin{array}{cc}
-\frac{1}{x_{i}-1}+\frac{x_{i+1}}{x_{i}-x_{i+1}} & \left(\frac{1}{x_{i}-1}-\frac{x_{i+1}}{x_{i}-x_{i+1}}\right)\left(-\frac{x_{i+1}}{x_{i}-x_{i+1}}+\frac{x_{i+1}}{1-x_{i+1}}\right)-\left(\frac{x_{i+1}}{x_{i}-x_{i+1}}\right)^{2} \\
1 & \frac{x_{i+1}}{x_{i}-x_{i+1}}-\frac{x_{i+1}}{1-x_{i+1}}
\end{array}\right), \\
= & \frac{x_{i+1}}{x_{i}}\left(\begin{array}{cc}
\frac{x_{i}\left(x_{i+1}-1\right)}{\left(x_{i}-1\right) x_{i+1}} & 1 \\
\frac{x_{i}-x_{i+1}}{x_{i+1}} & \frac{x_{i}-1}{x_{i+1}-1}
\end{array}\right)
\end{aligned}
$$

as in (44). Further, the contribution from the diagram

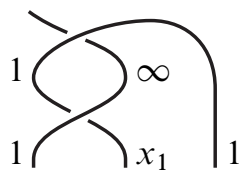

to the Hesse matrix is given by

$$
\left(\begin{array}{cc}
\frac{x_{1}}{1-x_{1}}+\cdots & \ddots \\
\ddots & \ddots
\end{array}\right)
$$

Hence we define $\Psi$ of this diagram by (42). Furthermore, by similar arguments as above, we define $\Psi$ of elementary diagrams of the bottom part by (45) and (46). For an open two-bridge knot diagram $D$ obtained by gluing copies of elementary diagrams, $\Psi(D)$ is defined to be the product of $\Psi$ of such elementary diagrams as in the statement of the lemma.

Therefore, by the above construction of $\Psi$, we have that

$$
\Psi(D)=\Omega_{1}(D) \Omega_{2}(D) \operatorname{det} H .
$$

Hence, from the definition of $\omega_{2}$, we obtain the required formula of the lemma. 


\section{Proof of Theorem 1.1}

In this section, we prove Theorem 1.1. We introduce $\check{\Phi}, \check{\Psi}$ and $\phi_{m}, \psi_{m}$ modifying $\widehat{\Phi}, \Psi$, and reduce the proof of the theorem to Proposition 5.1.

For an open two-bridge knot diagram $D$, we define $\breve{\Phi}(D)$ to be $\widehat{\Phi}$ of the diagram obtained from $D$ by $\pi$ rotation and by exchanging the positive and negative crossings:
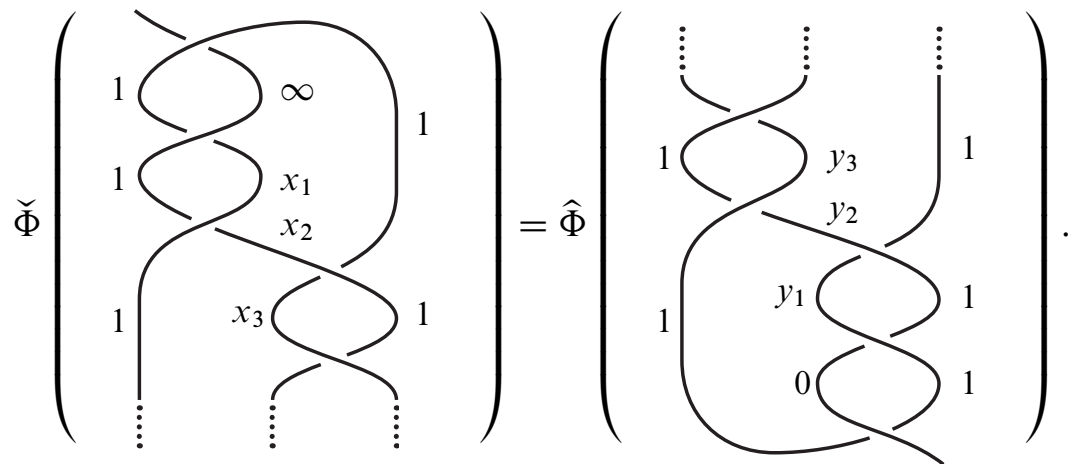

In other words, $\check{\Phi}$ is defined by the following formulas:
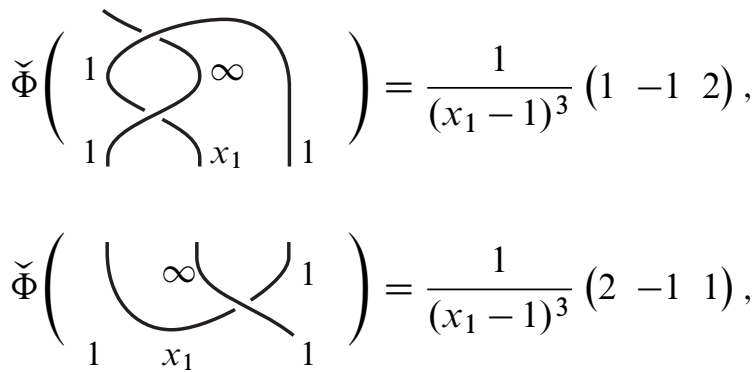

$\check{\Phi}\left(\left.\left.\int_{1}^{1}\right|_{x_{i+1}} ^{x_{i}}\right|_{1} ^{1}\right)=\frac{1}{x_{i}}\left(\begin{array}{ccc}1 & -1 & 1 \\ 0 & -\frac{1}{x_{i}} & \frac{2}{x_{i}} \\ 0 & 0 & 1\end{array}\right)$,

$\check{\Phi}\left(\begin{array}{c|c}1 & x_{i} \\ & \\ 1 & x_{i+1}\end{array} \sum_{1}^{1}{ }_{1}\right)=\frac{1}{x_{i}}\left(\begin{array}{ccc}1 & 0 & 0 \\ \frac{2}{x_{i}} & -\frac{1}{x_{i}} & 0 \\ 1 & -1 & 1\end{array}\right)$,

$\check{\Phi}\left(\begin{array}{c}1 \\ 0\end{array}\right)=\frac{x_{m-1}-1}{x_{m-1}^{2}}\left(\begin{array}{c}1 \\ \frac{2}{x_{m-1}} \\ 0\end{array}\right)$. 
Further, similarly as the calculation in Section 3, we can show that

$$
\left.\check{\Phi}\left({ }_{1}^{1} \bigcup^{x_{m-1}}\right)^{1}\right)=\frac{1-x_{m-1}}{x_{m-1}^{2}}\left(\begin{array}{c}
0 \\
\frac{2}{x_{m-1}} \\
1
\end{array}\right) .
$$

Without assuming that $x_{m}=0$, we can set

$$
\left.\check{\Phi}\left({ }^{1} \zeta^{x_{m}}\right)^{1}\right)=\check{\Phi}\left({ }^{1}\left(x_{m} \zeta^{1}\right)=\frac{x_{m-1}-1}{x_{m-1}}\left(\begin{array}{c}
1 \\
0 \\
-1
\end{array}\right)\right. \text {, }
$$

consistent with the above definition.

We define $\check{\Psi}$ by the formulas
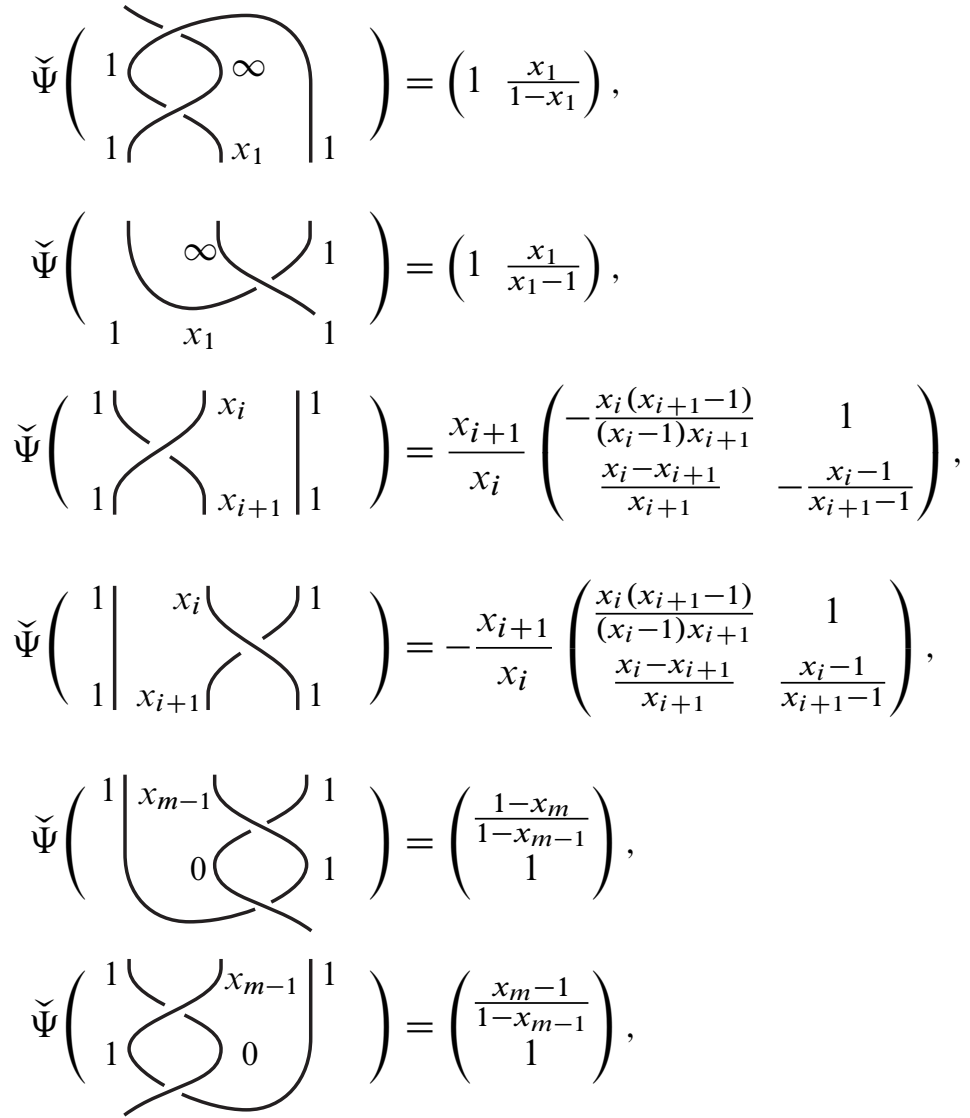

without assuming that $x_{m}=0$. When $x_{m}=0$, this definition is equal to the definition of $\Psi$ except for the sign of $\breve{\Psi}\left(\sigma_{2}^{-1}\right)$. Hence, $\Psi(D) \doteq \breve{\Psi}(D)$ when $x_{m}=0$. 
Without assuming that $x_{m}=0$, we can set

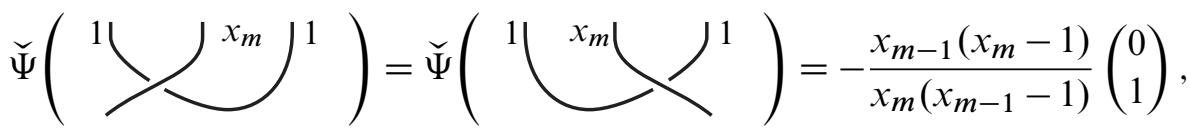

consistent with the above definition.

We recall that our diagram of an open two-bridge knot is a plat closure of a product of copies of $\sigma_{1}$ and $\sigma_{2}^{-1}$. By the hyperbolicity equations, the values of $x_{i}$ are recursively determined by (40). Putting $x_{1}=x$ (and $x_{0}=\infty$ ), we can regard $x_{i}$ as a rational function of $x$; we let it be $f_{i}(x)$. The hyperbolicity equation of the knot is given by $f_{m}(x)=0$.

Without assuming that $x_{m}=0$, we set

$$
\phi_{m}(x)=\left(x_{m}-1\right)^{2} \check{\Phi}(D), \quad \psi_{m}(x)=\frac{1-\frac{x_{m}}{x_{m}-1}}{1-x_{m}} \check{\Psi}(D),
$$

as rational functions of $x$.

Proof of Theorem 1.1 The required formula of the theorem is rewritten as

$$
\frac{2}{\tau(K)}=\frac{1}{\sqrt{-1} \omega_{2}(D)}
$$

for a diagram $D$ of an open two-bridge knot $K$. By (34), the left-hand side is equal to $\widehat{\Phi}(D)$. By Lemma 4.14, the right-hand side is equal to $\Psi(D)$. They are equal to $\breve{\Phi}(D)$ and $\breve{\Psi}(D)$ respectively, as we explained above. Further, they are equal to $\phi_{m}(c)$ and $\psi_{m}(c)$ respectively, for a root $x=c$ of $f_{m}(x)=0$. Since they are equal by Proposition 5.1 below, we obtain the required formula of the theorem.

As mentioned above, the proof of the theorem is reduced to the following proposition.

\section{Proposition 5.1}

$$
\phi_{m}(x)=\psi_{m}(x)
$$

Proof We recall that our diagram of an open two-bridge knot is a plat closure of a product of copies of $\sigma_{1}$ and $\sigma_{2}^{-1}$. We let the end of this product be $\cdots b_{3} b_{2} b_{1} b_{0}$, where $b_{3}, b_{2}, b_{1}, b_{0}=\sigma_{1}$ or $\sigma_{2}^{-1}$. We note that the knot type does not depend on the choice of $b_{0}$. Further, by the symmetry of Lemma 5.2 below, we can assume that $b_{1}=\sigma_{2}^{-1}$. In the following of this proof, we prove the proposition by induction on $m$, in the four cases of the choices of $b_{3}$ and $b_{2}$. The initial cases of the induction (the cases where $m \leq 3$ ) hold by Example 5.3 below. In the following proof, we show the required formula of the case of $m$, assuming the case of $m^{\prime}$ for $m^{\prime}<m$. 
We note that $f_{k}(x)$ is not equal to 1 . (Because, the equation $f_{k}(x)=0$ is the hyperbolicity equation of some two-bridge knot, which is a hyperbolic knot or the $(2, n)$ torus knot. In any case, $f_{k}(x)$ is a nontrivial rational function of $x$. In particular, it is not equal to 1 .)

We further note that $f_{k}(x)$ and $f_{k+1}(x)$ are not equal. (Because, if $f_{k}(x)$ and $f_{k+1}(x)$ were equal, we can show by the recursive formula of $x_{i}$ that $f_{j}(x)=1$ for some $j$, which contradicts the above claim.)

For simplicity, we denote $\phi_{i}(x), \psi_{i}(x), f_{i}(x)$ by $\phi_{i}, \psi_{i}, f_{i}$. By definition, $f_{i}=x_{i}$, without assuming that $x_{m}=0$. We set

$$
\begin{aligned}
& P_{i}=\frac{f_{i+1}}{f_{i}}\left(\begin{array}{cc}
-\frac{f_{i}\left(f_{i+1}-1\right)}{\left(f_{i}-1\right) f_{i+1}} & 1 \\
\frac{f_{i}-f_{i+1}}{f_{i+1}} & -\frac{f_{i}-1}{f_{i+1}-1}
\end{array}\right), \\
& Q_{i}=-\frac{f_{i+1}}{f_{i}}\left(\begin{array}{cc}
\frac{f_{i}\left(f_{i+1}-1\right)}{\left(f_{i}-1\right) f_{i+1}} & 1 \\
\frac{f_{i}-f_{i+1}}{f_{i+1}} & \frac{f_{i}-1}{f_{i+1}-1}
\end{array}\right) \text {, } \\
& v_{m}=\frac{f_{m-1}-f_{m}}{f_{m}\left(f_{m-1}-1\right)}\left(\begin{array}{l}
0 \\
1
\end{array}\right) \text {, } \\
& P_{i}^{\prime}=\frac{1}{f_{i}}\left(\begin{array}{ccc}
1 & -1 & 1 \\
0 & -\frac{1}{f_{i}} & \frac{2}{f_{i}} \\
0 & 0 & 1
\end{array}\right) \text {, } \\
& Q_{i}^{\prime}=\frac{1}{f_{i}}\left(\begin{array}{ccc}
1 & 0 & 0 \\
\frac{2}{f_{i}} & -\frac{1}{f_{i}} & 0 \\
1 & -1 & 1
\end{array}\right) \text {, } \\
& v_{m}^{\prime}=\frac{\left(f_{m-1}-1\right)\left(f_{m}-1\right)^{2}}{f_{m-1}}\left(\begin{array}{c}
1 \\
0 \\
-1
\end{array}\right) \text {. }
\end{aligned}
$$

By definition, $f_{i}$ satisfies the recursive formula (41). For simplicity, in the following of this proof, we write formulas of the case of $m=10$. (We can easily obtain the formulas of general $m$ from them by replacing $10,9,8,7$ with $m, m-1, m-2, m-3$.)

Case 1: $\boldsymbol{b}_{\mathbf{3}}=\boldsymbol{b}_{\mathbf{2}}=\boldsymbol{\sigma}_{\mathbf{2}}^{-1}$ In this case, we have that

$$
f_{10}=f_{9}+1-\frac{f_{9}}{f_{8}}, \quad f_{9}=f_{8}+1-\frac{f_{8}}{f_{7}} .
$$

In the definition of $\breve{\Psi}$, the differences among $\psi_{8}, \psi_{9}, \psi_{10}$ are presented by

$$
v_{8}, \quad Q_{8} v_{9}, \quad Q_{8} Q_{9} v_{10} .
$$


These vectors are linearly dependent. By calculating their coefficient concretely, we have that

$\frac{\left(f_{7}-1\right) f_{9}}{\left(f_{7}-f_{8}\right)\left(f_{9}-1\right)} \cdot v_{8}-\frac{f_{8}+f_{9}-f_{8} f_{9}-f_{8} f_{10}}{\left(f_{8}-f_{9}\right)\left(f_{10}-1\right)} \cdot Q_{8} v_{9}+\frac{f_{9}}{f_{9}-f_{10}} \cdot Q_{8} Q_{9} v_{10}=0$.

This is rewritten as the following linear relation among $\psi_{8}, \psi_{9}, \psi_{10}$ :

$$
\frac{\left(f_{7}-1\right) f_{9}}{\left(f_{7}-f_{8}\right)\left(f_{9}-1\right)} \cdot \psi_{8}-\frac{f_{8}+f_{9}-f_{8} f_{9}-f_{8} f_{10}}{\left(f_{8}-f_{9}\right)\left(f_{10}-1\right)} \cdot \psi_{9}+\frac{f_{9}}{f_{9}-f_{10}} \cdot \psi_{10}=0 .
$$

By (47), this is rewritten as

$$
\frac{f_{9}}{f_{8}} \psi_{8}-2 f_{8} \psi_{9}+f_{8} f_{9} \psi_{10}=0
$$

Similarly as above, we can show the following linear relation among $\psi_{7}, \psi_{8}, \psi_{9}$ :

$$
-\frac{\left(f_{6}-1\right)\left(f_{7}-f_{8}\right) f_{8}}{\left(f_{6}-f_{7}\right)\left(f_{8}-1\right)} \psi_{7}-2 f_{7} \psi_{8}+f_{7} f_{8} \psi_{9}=0
$$

noting that, in this case, we can not use $f_{8}=f_{7}+1-\frac{f_{7}}{f_{6}}$ at the present stage.

Similar to the case of $\check{\Phi}$, we show the following linear relation among $\phi_{7}, \phi_{8}, \phi_{9}, \phi_{10}$ :

$$
\begin{aligned}
& \frac{f_{6}\left(f_{9}-1\right)}{\left(f_{6}-1\right)\left(f_{7}-1\right)^{2} f_{7}} \phi_{7}+\frac{f_{7}\left(f_{9}-2\right)}{\left(f_{7}-1\right)\left(f_{8}-1\right)} \phi_{8} \\
& \quad-\frac{f_{8}^{2}\left(2 f_{8}-1\right)}{\left(f_{8}-1\right)\left(f_{9}-1\right)} \phi_{9}+\frac{f_{8} f_{9}^{3}\left(f_{8}-1\right)}{\left(f_{9}-1\right)\left(f_{10}-1\right)^{2}} \phi_{10}=0 .
\end{aligned}
$$

By (47), this is rewritten as

$$
\frac{f_{6} f_{8}\left(f_{8}-1\right)}{\left(f_{6}-1\right) f_{7}^{3}} \phi_{7}+\left(f_{9}-2\right) \phi_{8}-f_{8}\left(2 f_{8}-1\right) \phi_{9}+f_{8}^{2} f_{9} \phi_{10}=0 .
$$

Since $\psi_{7}=\phi_{7}, \psi_{8}=\phi_{8}, \psi_{9}=\phi_{9}$ by the assumption of the induction, we can eliminate $\phi_{7}, \psi_{8}, \phi_{8}, \psi_{9}, \phi_{9}$ by using (48), (49), (50). Then we obtain

$$
\left(\frac{\left(f_{6}-1\right)\left(f_{7}-f_{8}\right) f_{8}}{\left(f_{6}-f_{7}\right) f_{7}\left(f_{8}-1\right)}+\frac{f_{6} f_{8}\left(f_{8}-1\right)}{\left(f_{6}-1\right) f_{7}^{3}}\right) \psi_{7}=f_{8}^{2} f_{9}\left(\psi_{10}-\phi_{10}\right) .
$$

To show the proposition, it is sufficient to show that $\psi_{10}=\phi_{10}$. Hence, it is sufficient to show that

$$
\frac{\left(f_{6}-1\right)\left(f_{7}-f_{8}\right) f_{8}}{\left(f_{6}-f_{7}\right) f_{7}\left(f_{8}-1\right)}+\frac{f_{6} f_{8}\left(f_{8}-1\right)}{\left(f_{6}-1\right) f_{7}^{3}}=0 .
$$


This is rewritten as

$$
\left(-f_{8}+f_{7}+1-\frac{f_{7}}{f_{6}}\right)\left(-f_{8}+f_{7}+\frac{\left(f_{7}-1\right)^{2}}{1-f_{7} / f_{6}}\right)=0,
$$

which holds by the recursive formula of $f_{i}$. Therefore, we obtain the proposition in this case.

Case 2: $b_{3}=\sigma_{1}$ and $b_{2}=\sigma_{2}^{-1}$ In this case, we have that

$$
f_{10}=f_{9}+1-\frac{f_{9}}{f_{8}}, \quad f_{9}=f_{8}+\frac{\left(f_{8}-1\right)^{2}}{1-f_{8} / f_{7}} .
$$

In the definition of $\breve{\Psi}$, the differences among $\psi_{8}, \psi_{9}, \psi_{10}$ are presented by

$$
v_{8}, \quad Q_{8} v_{9}, \quad Q_{8} Q_{9} v_{10} .
$$

These vectors are linearly dependent. By calculating their coefficient concretely, similarly as in Case 1 , we have that

$$
\frac{\left(f_{7}-1\right) f_{9}}{\left(f_{7}-f_{8}\right)\left(f_{9}-1\right)} \psi_{8}-\frac{f_{8}+f_{9}-f_{8} f_{9}-f_{8} f_{10}}{\left(f_{8}-f_{9}\right)\left(f_{10}-1\right)} \psi_{9}+\frac{f_{9}}{f_{9}-f_{10}} \psi_{10}=0 .
$$

By (52), this is rewritten as

$$
\frac{f_{7}\left(f_{8}-1\right)}{f_{8}\left(f_{7}-f_{8}\right)} \psi_{8}-\frac{2 f_{8}}{f_{9}} \psi_{9}+f_{8} \psi_{10}=0
$$

Similarly, by calculating the coefficients of the linear dependence among

$$
v_{7}, \quad P_{7} v_{8}, \quad P_{7} Q_{8} v_{9}
$$

we have that

$$
-\frac{\left(f_{6}-1\right) f_{8}}{\left(f_{6}-f_{7}\right)\left(f_{8}-1\right)} \psi_{7}+\frac{f_{7}-f_{8}+f_{7} f_{8}-f_{7} f_{9}}{\left(f_{7}-f_{8}\right)\left(f_{9}-1\right)} \psi_{8}+\frac{f_{8}}{f_{8}-f_{9}} \psi_{9}=0 .
$$

By (52), this is rewritten as

$$
\frac{f_{6}-1}{f_{6}-f_{7}} \psi_{7}+\frac{f_{7} f_{8}-2 f_{7}+f_{8}}{\left(f_{7}-f_{8}\right) f_{8}} \psi_{8}+\frac{f_{7}-f_{8}}{f_{7}\left(f_{8}-1\right)} \psi_{9}=0 .
$$

Further, in the definition of $\check{\Phi}$, by calculating the coefficients of the linear dependence among

$$
v_{7}^{\prime}, \quad P_{7}^{\prime} v_{8}^{\prime}, \quad P_{7}^{\prime} Q_{8}^{\prime} v_{9}^{\prime}, \quad P_{7}^{\prime} Q_{8}^{\prime} Q_{9}^{\prime} v_{10}^{\prime},
$$


we have that

$$
\begin{aligned}
& \frac{f_{6}\left(f_{9}-1\right)}{f_{7}\left(f_{6}-1\right)\left(f_{7}-1\right)^{2}} \phi_{7}-\frac{f_{7}\left(1-2 f_{8}+f_{8} f_{9}\right)}{\left(f_{7}-1\right)\left(f_{8}-1\right)^{2}} \phi_{8} \\
& \quad+\frac{f_{8}^{2}\left(2 f_{8}-1\right)}{\left(f_{8}-1\right)\left(f_{9}-1\right)} \phi_{9}-\frac{f_{8} f_{9}^{3}\left(f_{8}-1\right)}{\left(f_{9}-1\right)\left(f_{10}-1\right)^{2}} \phi_{10}=0 .
\end{aligned}
$$

By (52), this is rewritten as

$$
\frac{f_{6}\left(f_{8}-1\right)^{3}}{f_{7}\left(f_{6}-1\right)\left(f_{7}-f_{8}\right)^{2}} \phi_{7}-\frac{f_{7}\left(1-2 f_{8}+f_{8} f_{9}\right)}{f_{8}\left(f_{7}-f_{8}\right)} \phi_{8}+\left(2 f_{8}-1\right) \phi_{9}-f_{8} f_{9} \phi_{10}=0 .
$$

Since $\psi_{7}=\phi_{7}, \psi_{8}=\phi_{8}, \psi_{9}=\phi_{9}$ by the assumption of the induction, we obtain the following relation from (53) and (55):

$$
\frac{f_{6}\left(f_{8}-1\right)^{3}}{f_{7}\left(f_{6}-1\right)\left(f_{7}-f_{8}\right)^{2}} \psi_{7}-\frac{f_{7}\left(1-2 f_{8}+f_{9}\right)}{f_{8}\left(f_{7}-f_{8}\right)} \psi_{8}-\psi_{9}=f_{8} f_{9}\left(\phi_{10}-\psi_{10}\right) .
$$

To show the proposition, it is sufficient to show that $\psi_{10}=\phi_{10}$. Hence, it is sufficient to show that

$$
\frac{f_{6}\left(f_{8}-1\right)^{3}}{f_{7}\left(f_{6}-1\right)\left(f_{7}-f_{8}\right)^{2}} \psi_{7}-\frac{f_{7}\left(1-2 f_{8}+f_{9}\right)}{f_{8}\left(f_{7}-f_{8}\right)} \psi_{8}-\psi_{9}=0 .
$$

By using (52), this is rewritten as

$$
\frac{f_{6}\left(f_{8}-1\right)^{3}}{f_{7}\left(f_{6}-1\right)\left(f_{7}-f_{8}\right)^{2}} \psi_{7}-\frac{f_{7}\left(f_{8}-1\right)\left(f_{7} f_{8}-2 f_{7}-f_{8}\right)}{f_{8}\left(f_{7}-f_{8}\right)^{2}} \psi_{8}-\psi_{9}=0 .
$$

Further, by using (54), we can eliminate $\psi_{8}$ and $\psi_{9}$. Then we obtain

$$
\left(\frac{f_{6}\left(f_{8}-1\right)^{3}}{f_{7}\left(f_{6}-1\right)\left(f_{7}-f_{8}\right)^{2}}+\frac{\left(f_{6}-1\right) f_{7}\left(f_{8}-1\right)}{\left(f_{6}-f_{7}\right)\left(f_{7}-f_{8}\right)}\right) \psi_{7}=0 .
$$

Since the coefficient is $\psi_{7}$ is rewritten as (51), this formula holds similarly as in Case 1 . Therefore, we obtain the proposition in this case.

Case 3: $b_{3}=b_{2}=\sigma_{1}$ In this case, we have that

$$
f_{10}=f_{9}+\frac{\left(f_{9}-1\right)^{2}}{1-f_{9} / f_{8}}, \quad f_{9}=f_{8}+1-\frac{f_{8}}{f_{7}} .
$$

In the definition of $\breve{\Psi}$, the differences among $\psi_{8}, \psi_{9}, \psi_{10}$ are presented by

$$
v_{8}, \quad P_{8} v_{9}, \quad P_{8} Q_{9} v_{10} \text {. }
$$


These vectors are linearly dependent. By calculating their coefficient concretely, similarly as in Case 1, we have that

$$
-\frac{\left(f_{7}-1\right) f_{9}}{\left(f_{7}-f_{8}\right)\left(f_{9}-1\right)} \psi_{8}-\frac{f_{8}-f_{9}+f_{8} f_{9}-f_{8} f_{10}}{\left(f_{8}-f_{9}\right)\left(f_{10}-1\right)} \psi_{9}+\frac{f_{9}}{f_{9}-f_{10}} \psi_{10}=0 .
$$

By (56), this is rewritten as

$$
-\frac{f_{9}}{f_{8}} \psi_{8}+\frac{f_{8} f_{9}-2 f_{8}+f_{9}}{f_{9}-1} \psi_{9}+\frac{f_{9}\left(f_{8}-f_{9}\right)^{2}}{f_{8}\left(f_{9}-1\right)^{2}} \psi_{10}=0 .
$$

Similarly, by calculating the coefficients of the linear dependence among

$$
v_{7}, \quad P_{7} v_{8}, \quad P_{7} P_{8} v_{9}
$$

we have that

$$
\frac{\left(f_{6}-1\right) f_{8}}{\left(f_{6}-f_{7}\right)\left(f_{8}-1\right)} \psi_{7}+\frac{-f_{7}-f_{8}+f_{7} f_{8}+f_{7} f_{9}}{\left(f_{7}-f_{8}\right)\left(f_{9}-1\right)} \psi_{8}+\frac{f_{8}}{f_{8}-f_{9}} \psi_{9}=0 .
$$

By (56), this is rewritten as

$$
\frac{\left(f_{6}-1\right)\left(f_{7}-f_{8}\right)}{\left(f_{6}-f_{7}\right) f_{7}\left(f_{8}-1\right)} \psi_{7}+\frac{2}{f_{8}} \psi_{8}-\psi_{9}=0 .
$$

Further, in the definition of $\breve{\Phi}$, by calculating the coefficients of the linear dependence among

$$
v_{7}^{\prime}, \quad P_{7}^{\prime} v_{8}^{\prime}, \quad P_{7}^{\prime} P_{8}^{\prime} v_{9}^{\prime}, \quad P_{7}^{\prime} P_{8}^{\prime} Q_{9}^{\prime} v_{10}^{\prime},
$$

we have that

$$
\begin{aligned}
-\frac{f_{6}\left(f_{9}-1\right)}{\left(f_{6}-1\right) f_{7}\left(f_{7}-1\right)^{2}\left(f_{8}-1\right)} & \phi_{7}-\frac{f_{7}\left(f_{9}-2\right)}{\left(f_{7}-1\right)\left(f_{8}-1\right)^{2}} \phi_{8} \\
+ & \frac{f_{8}^{2}\left(1-2 f_{8}+f_{8} f_{9}\right)}{\left(f_{8}-1\right)^{2}\left(f_{9}-1\right)^{2}} \phi_{9}+\frac{f_{8} f_{9}^{3}}{\left(f_{9}-1\right)\left(f_{10}-1\right)^{2}} \phi_{10}=0 .
\end{aligned}
$$

By (56), this is rewritten as

$$
-\frac{f_{6}\left(f_{8}-1\right)}{f_{7}^{3}\left(f_{6}-1\right)} \phi_{7}-\frac{f_{9}-2}{f_{8}} \phi_{8}+\frac{1-2 f_{8}+f_{8} f_{9}}{f_{9}-1} \phi_{9}+\frac{f_{9}\left(f_{8}-f_{9}\right)^{2}}{f_{8}\left(f_{9}-1\right)^{2}} \phi_{10}=0 .
$$

Since $\psi_{7}=\phi_{7}, \psi_{8}=\phi_{8}, \psi_{9}=\phi_{9}$ by the assumption of the induction, we obtain the following relation from (57) and (59),

$$
\frac{f_{6}\left(f_{8}-1\right)}{f_{7}^{3}\left(f_{6}-1\right)} \psi_{7}-\frac{2}{f_{8}} \psi_{8}+\psi_{9}=\frac{f_{9}\left(f_{8}-f_{9}\right)^{2}}{f_{8}\left(f_{9}-1\right)^{2}}\left(\phi_{10}-\psi_{10}\right) .
$$


To show the proposition, it is sufficient to show $\psi_{10}=\phi_{10}$. Hence, it is sufficient to show

$$
\frac{f_{6}\left(f_{8}-1\right)}{f_{7}^{3}\left(f_{6}-1\right)} \psi_{7}-\frac{2}{f_{8}} \psi_{8}+\psi_{9}=0 .
$$

By this formula and (58), we can eliminate $\psi_{8}$ and $\psi_{9}$. Then we obtain

$$
\left(\frac{f_{6}\left(f_{8}-1\right)}{f_{7}^{3}\left(f_{6}-1\right)}+\frac{\left(f_{6}-1\right)\left(f_{7}-f_{8}\right)}{\left(f_{6}-f_{7}\right) f_{7}\left(f_{8}-1\right)}\right) \psi_{7}=0 .
$$

Since the coefficient of $\psi_{7}$ is rewritten as (51), this formula holds similarly as in Case 1 . Therefore, we obtain the proposition in this case.

Case 4: $b_{3}=\sigma_{2}^{-1}$ and $b_{2}=\sigma_{1}$ In this case, we have that

$$
f_{10}=f_{9}+\frac{\left(f_{9}-1\right)^{2}}{1-f_{9} / f_{8}}, \quad f_{9}=f_{8}+\frac{\left(f_{8}-1\right)^{2}}{1-f_{8} / f_{7}} .
$$

We set

$$
\psi_{k}^{\prime}=\frac{\psi_{k}}{\left(x_{k-1}-1\right)\left(x_{k}-1\right)^{2}}, \quad \phi_{k}^{\prime}=\frac{\phi_{k}}{\left(x_{k-1}-1\right)\left(x_{k}-1\right)^{2}}
$$

for $k=7,8,9,10$.

In the definition of $\breve{\Psi}$, the differences among $\psi_{8}, \psi_{9}, \psi_{10}$ are the same as in Case 3, and we have that

$$
-\frac{\left(f_{7}-1\right) f_{9}}{\left(f_{7}-f_{8}\right)\left(f_{9}-1\right)} \psi_{8}-\frac{f_{8}-f_{9}+f_{8} f_{9}-f_{8} f_{10}}{\left(f_{8}-f_{9}\right)\left(f_{10}-1\right)} \psi_{9}+\frac{f_{9}}{f_{9}-f_{10}} \psi_{10}=0 .
$$

By replacing $\psi_{k}$ with $\psi_{k}^{\prime}$ and by using (60), this is rewritten as

$$
f_{7} f_{9}\left(f_{8}-1\right) \psi_{8}^{\prime}-f_{8}^{2}\left(-2 f_{8}+f_{9}+f_{8} f_{9}\right) \psi_{9}^{\prime}-f_{8} f_{9}^{3}\left(f_{8}-1\right) \psi_{10}^{\prime}=0 .
$$

Similarly, by calculating the coefficients of the linear dependence among

$$
v_{7}, \quad Q_{7} v_{8}, \quad Q_{7} P_{8} v_{9}
$$

we have that

$$
-\frac{\left(f_{6}-1\right) f_{8}\left(f_{9}-1\right)}{\left(f_{6}-f_{7}\right)\left(f_{8}-1\right)} \psi_{7}+\frac{f_{7}-f_{8}+f_{7} f_{8}-f_{7} f_{9}}{f_{7}-f_{8}} \psi_{8}+\frac{f_{8}\left(f_{9}-1\right)}{f_{8}-f_{9}} \psi_{9}=0 .
$$


By replacing $\psi_{k}$ with $\psi_{k}^{\prime}$ and by using (60), this is rewritten as

$$
\begin{aligned}
\frac{\left(f_{6}-1\right)^{2}\left(f_{7}-1\right)^{2} f_{8}^{2}}{\left(f_{6}-f_{7}\right)\left(f_{8}-1\right)\left(f_{9}-1\right)} \psi_{7}^{\prime}+\left(-2 f_{7}+f_{8}\right. & \left.+f_{7} f_{8}\right) \psi_{8}^{\prime} \\
& +\frac{f_{8}^{2}\left(f_{7}-f_{8}\right)\left(f_{9}-1\right)}{f_{7}\left(f_{8}-1\right)} \psi_{9}^{\prime}=0 .
\end{aligned}
$$

Further, in the definition of $\breve{\Phi}$, by calculating the coefficients of the linear dependence among

$$
v_{7}^{\prime}, \quad Q_{7}^{\prime} v_{8}^{\prime}, \quad Q_{7}^{\prime} P_{8}^{\prime} v_{9}^{\prime}, \quad Q_{7}^{\prime} P_{8}^{\prime} Q_{9}^{\prime} v_{10}^{\prime},
$$

we have that

$$
\begin{aligned}
\frac{f_{6}\left(f_{9}-1\right)}{f_{7}\left(f_{6}-1\right)\left(f_{7}-1\right)^{2}} \phi_{7}- & \frac{f_{7}\left(1-2 f_{8}+f_{8} f_{9}\right)}{\left(f_{7}-1\right)\left(f_{8}-1\right)^{2}} \phi_{8} \\
& +\frac{f_{8}^{2}\left(1-2 f_{8}+f_{8} f_{9}\right)}{\left(f_{8}-1\right)\left(f_{9}-1\right)^{2}} \phi_{9}+\frac{f_{8} f_{9}^{3}\left(f_{8}-1\right)}{\left(f_{9}-1\right)\left(f_{10}-1\right)^{2}} \phi_{10}=0 .
\end{aligned}
$$

By replacing $\phi_{k}$ with $\phi_{k}^{\prime}$ and by using (60), this is rewritten as

$$
\begin{aligned}
\frac{f_{6}\left(f_{9}-1\right)}{f_{7}} \phi_{7}^{\prime}-f_{7}\left(1-2 f_{8}+f_{8} f_{9}\right) \phi_{8}^{\prime}+f_{8}^{2}\left(1-2 f_{8}\right. & \left.+f_{8} f_{9}\right) \phi_{9}^{\prime} \\
& +f_{8} f_{9}^{3}\left(f_{8}-1\right) \phi_{10}^{\prime}=0
\end{aligned}
$$

Since $\psi_{7}^{\prime}=\phi_{7}^{\prime}, \psi_{8}^{\prime}=\phi_{8}^{\prime}, \psi_{9}^{\prime}=\phi_{9}^{\prime}$ by the assumption of the induction, we obtain the following relation from (61) and (63):

$$
\frac{f_{6}\left(f_{9}-1\right)}{f_{7}} \psi_{7}^{\prime}-f_{7}\left(1-2 f_{8}+f_{9}\right) \psi_{8}^{\prime}-f_{8}^{2}\left(f_{9}-1\right) \psi_{9}^{\prime}=f_{8} f_{9}^{3}\left(f_{8}-1\right)\left(\psi_{10}^{\prime}-\phi_{10}^{\prime}\right) .
$$

To show the proposition, it is sufficient to show that $\psi_{10}=\phi_{10}$. Hence, it is sufficient to show that

$$
\frac{f_{6}\left(f_{9}-1\right)}{f_{7}} \psi_{7}^{\prime}-f_{7}\left(1-2 f_{8}+f_{9}\right) \psi_{8}^{\prime}-f_{8}^{2}\left(f_{9}-1\right) \psi_{9}^{\prime}=0 .
$$

By (60), this is rewritten as

$$
\frac{f_{6}\left(f_{7}-f_{8}\right)\left(f_{9}-1\right)}{f_{7}^{2}\left(f_{8}-1\right)} \psi_{7}^{\prime}-\left(-2 f_{7}+f_{8}+f_{7} f_{8}\right) \psi_{8}^{\prime}-\frac{f_{8}^{2}\left(f_{7}-f_{8}\right)\left(f_{9}-1\right)}{f_{7}\left(f_{8}-1\right)} \psi_{9}^{\prime}=0 .
$$


By this formula and (62), we can eliminate $\psi_{8}^{\prime}$ and $\psi_{9}^{\prime}$. Then we obtain

$$
\left(\frac{\left(f_{6}-1\right)^{2}\left(f_{7}-1\right)^{2} f_{8}^{2}}{\left(f_{6}-f_{7}\right)\left(f_{8}-1\right)\left(f_{9}-1\right)}+\frac{f_{6}\left(f_{7}-f_{8}\right)\left(f_{9}-1\right)}{f_{7}^{2}\left(f_{8}-1\right)}\right) \psi_{7}^{\prime}=0 .
$$

Since the coefficient of $\psi_{7}$ is rewritten as (51), this formula holds similarly as in Case 1. Therefore, we obtain the proposition in this case.

The following lemma is used in the proof of the above proposition.

Lemma 5.2 By the reflection of an open two-bridge knot diagram with respect to a vertical line, $\sigma_{1}$ and $\sigma_{2}^{-1}$ are exchanged, and the values of $\phi_{m}$ and $\psi_{m}$ become $(-1)$-multiples of the original values.

Proof In the definition of $\check{\Phi}, \breve{\Phi}\left(\sigma_{1}\right)$ and $\check{\Phi}\left(\sigma_{2}^{-1}\right)$ are conjugate by

$$
M_{1}=\left(\begin{array}{lll}
0 & 0 & 1 \\
0 & 1 & 0 \\
1 & 0 & 0
\end{array}\right) \text {. }
$$

Further, the value of $\check{\Phi}$ of the top part of a two-bridge knot diagram becomes $M_{1}-$ multiple of the original vector. Furthermore, the value of $\check{\Phi}$ of the bottom part of a two-bridge knot diagram becomes $\left(-M_{1}\right)$-multiple of the original vector. Hence, the value of $\phi_{m}$ becomes a $(-1)$-multiple of the original value.

In the definition of $\breve{\Psi}, \breve{\Psi}\left(\sigma_{1}\right)$ and $\breve{\Phi}\left(\sigma_{2}^{-1}\right)$ are conjugate by

$$
M_{2}=\left(\begin{array}{rr}
1 & 0 \\
0 & -1
\end{array}\right) \text {. }
$$

Further, the value of $\check{\Psi}$ of the top part of a two-bridge knot diagram becomes $M_{2}-$ multiple of the original vector. Furthermore, the value of $\breve{\Psi}$ of the bottom part of a two-bridge knot diagram becomes $\left(-M_{2}\right)$-multiple of the original vector. Hence, the value of $\psi_{m}$ becomes $(-1)$-multiple of the original value, as required.

Without assuming that $x_{0}=\infty$, we can set

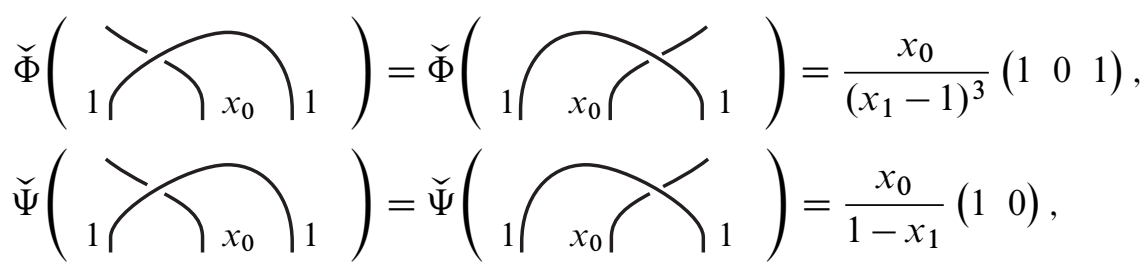


consistently with the definitions of $\check{\Phi}$ and $\breve{\Psi}$. Indeed, we can show Lemma 5.2 without using these formulas, but these formulas are helpful to understand the symmetry of Lemma 5.2.

In the following example, we show the initial cases of the induction of the proof of Proposition 5.1.

Example 5.3 Proposition 5.1 holds for $m \leq 3$.

Proof By the symmetry of Lemma 5.2, it suffices to show the formula of Proposition 5.1 for the plat closures of $\sigma_{1}^{2} \cdot b \cdot b_{0}$ for $b=1, \sigma_{1}, \sigma_{2}^{-1}, \sigma_{1}^{2}, \sigma_{1} \sigma_{2}^{-1}, \sigma_{2}^{-1} \sigma_{1}, \sigma_{2}^{-2}$, recalling that the knot type does not depend on the choice of $b_{0}=\sigma_{1}$ or $\sigma_{2}^{-1}$. We note that some of them are not knots, but 2-component links. We calculate both sides of the formula concretely for these cases.

For the plat closure of $\sigma_{1}^{2} \cdot b_{0}$,

$$
\phi_{1}(x)=\psi_{1}(x)=-\frac{1}{x-1} .
$$

For the plat closure of $\sigma_{1}^{2} \cdot \sigma_{1} \cdot b_{0}$,

$$
\phi_{2}(x)=\psi_{2}(x)=-\frac{2}{x(x-1)} .
$$

For the plat closure of $\sigma_{1}^{2} \cdot \sigma_{2}^{-1} \cdot b_{0}$,

$$
\phi_{2}(x)=\psi_{2}(x)=\frac{x-2}{x} .
$$

For the plat closure of $\sigma_{1}^{2} \cdot \sigma_{1}^{2} \cdot b_{0}$,

$$
\phi_{3}(x)=\psi_{3}(x)=-\frac{3 x-1}{x^{2}(x-1)(x+1)} .
$$

For the plat closure of $\sigma_{1}^{2} \cdot \sigma_{1} \sigma_{2}^{-1} \cdot b_{0}$,

$$
\phi_{3}(x)=\psi_{3}(x)=\frac{x\left(x^{2}-x+2\right)}{(x-1)(x+1)} .
$$

For the plat closure of $\sigma_{1}^{2} \cdot \sigma_{2}^{-1} \sigma_{1} \cdot b_{0}$,

$$
\phi_{3}(x)=\psi_{3}(x)=-\frac{2 x(x+1)}{x^{2}-x+1} .
$$


For the plat closure of $\sigma_{1}^{2} \cdot \sigma_{2}^{-2} \cdot b_{0}$,

$$
\phi_{3}(x)=\psi_{3}(x)=\frac{3 x^{2}-5 x+1}{x^{2}\left(x^{2}-x+1\right)} .
$$

Hence, Proposition 5.1 holds for $m \leq 3$.

\section{Appendix A: Cellular decompositions of the knot complement}

In this appendix, we explain that the cellular decomposition of the knot complement given in Step 2 of Section 3.2 is a modification of the dual of the ideal tetrahedral decomposition of $[25 ; 31]$ mentioned in Section 3.1. We explain this for the $\overline{5_{2}}$ knot.

We briefly review the ideal tetrahedral decomposition of the $\overline{5_{2}}$ knot; for details on this, see $[25 ; 31]$. As mentioned in Section 3.1, we consider 4 tetrahedra at each crossing, and we obtain the knot complement by gluing them; see the left picture below, where we show tetrahedra by triangles. As shown in [25; 31], this tetrahedral decomposition has one ideal vertex and two ordinary vertices. Further, as in $[25 ; 31]$, we collapse a tetrahedron of each dark gray triangle to an interval, and collapse a tetrahedron of each light gray triangle to a triangle. Then we obtain the ideal tetrahedral decomposition of $[25 ; 31]$, which describes the complete hyperbolic structure of the knot complement:
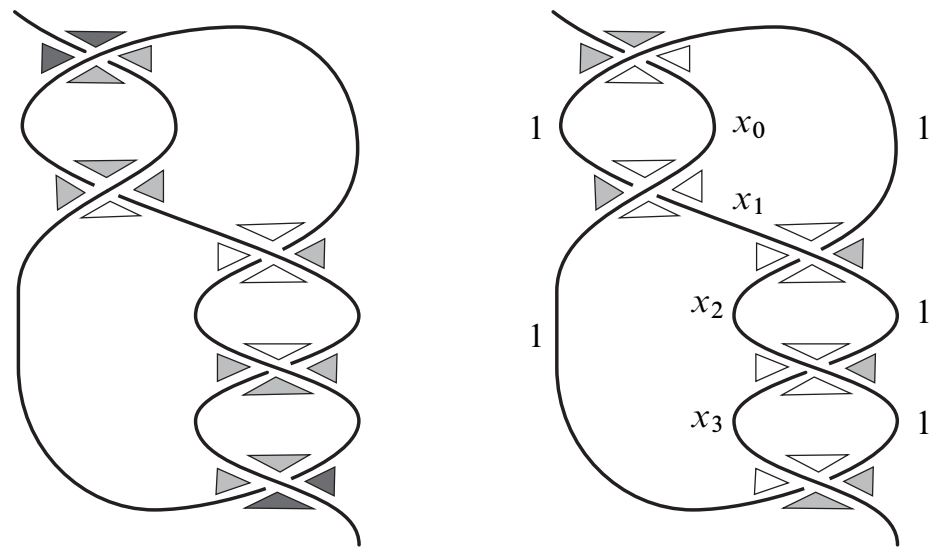

We consider to modify this collapsing, as in the right picture above, ie instead of collapsing of the left picture, we collapse a tetrahedron of each gray triangle of the right picture to a triangle. Then we obtain a tetrahedral decomposition $\mathcal{T}$ with one ideal vertex and one ordinary vertex. These vertices are connected by a single edge $e$. Further, as in (13), we glue tetrahedra at each region of the knot diagram. Then we 
obtain the following five (singular) polyhedra:
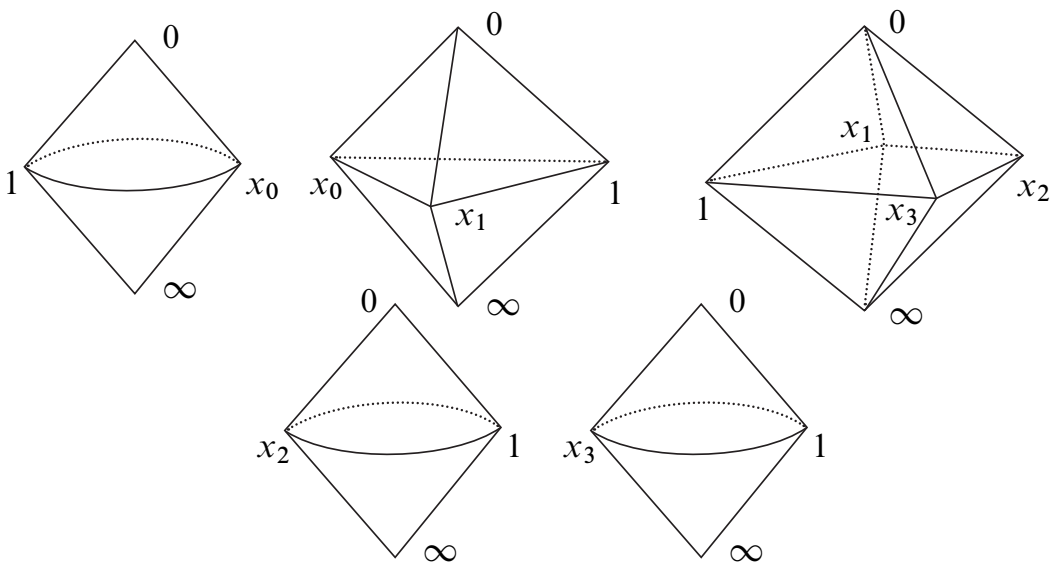

By regarding these polyhedra as 3-cells, we consider a cellular decomposition $\mathcal{T}^{\prime}$ as a modification of $\mathcal{T}$. Further, we consider the dual decomposition $\overline{\mathcal{T}}^{\prime}$ of $\mathcal{T}^{\prime}$. This decomposition has a 3-cell as the dual of the ordinary vertex of $\mathcal{T}^{\prime}$. By collapsing this 3-cell along the edge $e$, we obtain a cellular decomposition $\mathcal{T}^{\prime \prime}$ with no 3-cell. The 1 -cells of $\mathcal{T}^{\prime \prime}$ are the arrows of the following picture, and the 0 -cells are the gray regions.

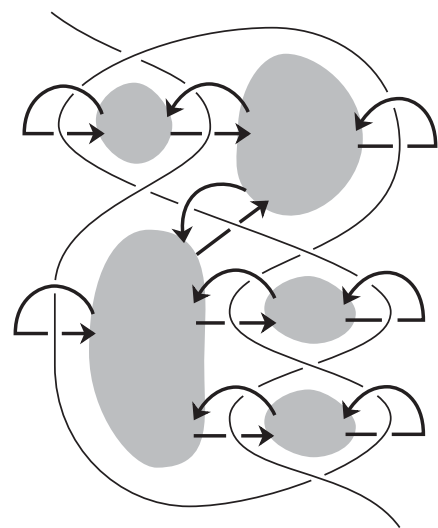

By connecting these 0 -cells along $X_{i}^{\prime}$, we obtain the cellular decomposition of Step 2 of Section 3.2 from $\mathcal{T}^{\prime \prime}$.

\section{Appendix B: Transformation of a basis of $C^{*}$}

In Section 3, we transform a basis of the chain complex $C^{*}$ to find a subcomplex $\widehat{C}^{*}$ of $C^{*}$. In this appendix, we explain a motivation of this transformation. 
We explain the motivation, for example, for the basis $e_{X_{i}}, e_{W_{i}}, e_{Z_{i}}$ of $X_{i}, W_{i}, Z_{i}$ given in (25). We recall that the knot group is generated by meridians, which are taken to $X_{i} X_{i}^{\prime}, W_{i}, Z_{i}$ by the monodromy representation, in this case. By the construction of these matrices, they are parabolic, and each of them fixes the value of the hyperbolicity parameter of the corresponding strand by the Möbius transformation. For example, $X_{i} X_{i}^{\prime}$ fixes $x_{i}$, and hence, the 1-eigenvector of $X_{i} X_{i}^{\prime}$ is given by

$$
\left(\begin{array}{c}
x_{i} \\
1
\end{array}\right) \text {. }
$$

Therefore, the following conjugation of $X_{i} X_{i}^{\prime}$ is equivalent (in $\mathrm{PGL}_{2} \mathbb{C}$ ) to an uppertriangular parabolic matrix,

$$
\left(\begin{array}{cc}
x_{i} & 1 \\
1 & 0
\end{array}\right)^{-1} X_{i} X_{i}^{\prime}\left(\begin{array}{cc}
x_{i} & 1 \\
1 & 0
\end{array}\right) \sim\left(\begin{array}{ll}
1 & * \\
0 & 1
\end{array}\right) .
$$

In similar ways, we have that

$$
\left(\begin{array}{ll}
1 & 1 \\
1 & 0
\end{array}\right)^{-1} W_{i}\left(\begin{array}{ll}
1 & 1 \\
1 & 0
\end{array}\right) \sim\left(\begin{array}{ll}
1 & 1 \\
0 & 1
\end{array}\right), \quad\left(\begin{array}{ll}
1 & 1 \\
1 & 0
\end{array}\right)^{-1} Z_{i}\left(\begin{array}{ll}
1 & 1 \\
1 & 0
\end{array}\right) \sim\left(\begin{array}{rr}
1 & -1 \\
0 & 1
\end{array}\right) .
$$

In such a way, we consider to transform a basis $e_{X_{i}}, e_{W_{i}}, e_{Z_{i}}$ to

$$
e_{X_{i}}^{\prime}=\operatorname{ad}\left(\begin{array}{cc}
x_{i} & 1 \\
1 & 0
\end{array}\right)^{-1} e_{X_{i}}, \quad e_{W_{i}}^{\prime}=\operatorname{ad}\left(\begin{array}{ll}
1 & 1 \\
1 & 0
\end{array}\right)^{-1} e_{W_{i}}, \quad e_{Z_{i}}^{\prime}=\operatorname{ad}\left(\begin{array}{ll}
1 & 1 \\
1 & 0
\end{array}\right)^{-1} e_{Z_{i}},
$$

expecting that this new basis gives a better description of $C^{*}$. Then the coboundary operator $D_{d}$ is transformed to the modified $D_{d}$ as we mentioned in Section 3, and we can find a subcomplex $\widehat{C}^{*}$ with respect to this modified $D_{d}$ as we showed by concrete calculation in Section 3.

\section{Appendix C: The numerators and the denominators of the $f_{i}$}

In Section 4.3, for a given two-bridge knot diagram, we consider hyperbolicity parameters $x_{i}$ as a rational function of $x$ determined by (40), and define it to be $f_{i}$. In this appendix, we characterize the numerator and the denominator of $f_{i}$ by a recursive formula. We set $x=u+1$, and regard $f_{i}$ as a rational function of $u$ in this appendix. We will use results of this appendix in Appendix D.

We explain how we formulate such a recursive formula by using the open two-bridge knot diagram below as an example. For this diagram, the hyperbolicity parameters $f_{i}$ 
are determined by (40), as follows:

$$
\begin{aligned}
& f_{1}=u+1, \\
& f_{2}=u+2, \\
& f_{3}=-u^{3}-3 u^{2}-2 u+1, \\
& f_{4}=-\frac{\left(u^{2}+u-1\right)\left(u^{2}+3 u+3\right)}{u+2}, \\
& f_{5}=\frac{u^{8}+7 u^{7}+19 u^{6}+22 u^{5}+3 u^{4}-14 u^{3}-6 u^{2}+4 u+1}{u+1} .
\end{aligned}
$$

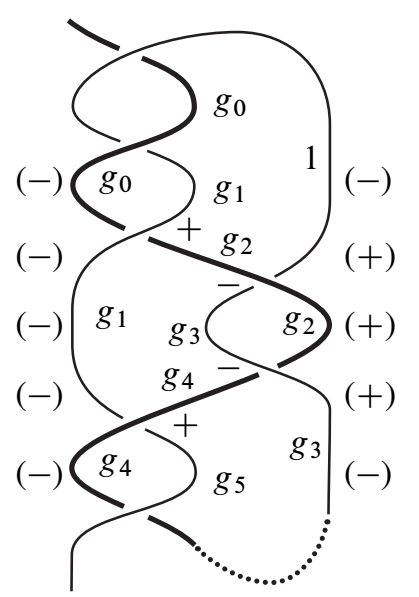

We set ${ }^{8}$ the numerator of $f_{i}$ to be $g_{i}$, and associate $g_{i}$ to the strand of $f_{i}$. Further, when two strands are connected by an overpath, we associate the same parameter to them. For the above diagram, the values of $g_{i}$ are given by

$$
\begin{aligned}
& g_{1}=u+1, \\
& g_{2}=u+2, \\
& g_{3}=u^{3}+3 u^{2}+2 u-1, \\
& g_{4}=\left(u^{2}+u-1\right)\left(u^{2}+3 u+3\right), \\
& g_{5}=u^{8}+7 u^{7}+19 u^{6}+22 u^{5}+3 u^{4}-14 u^{3}-6 u^{2}+4 u+1 .
\end{aligned}
$$

We set $g_{0}=1$. Further, as shown in (1), we depict the strand of the upper end by a thick line. Furthermore, we associate a sign to each crossing, and associate parenthesized signs to the left and right edges as in (1); we determine these signs in the following way.

${ }^{8}$ We redefine $g_{i}$ by a recursive formula (4) later, and show that $g_{i}$ gives the numerator of $f_{i}$ in Lemma C.2. 
We express an open two-bridge knot diagram as a plat closure of a product of copies of $\sigma_{1}$ and $\sigma_{2}^{-1}$. We give signs to each copy of $\sigma_{1}$ or $\sigma_{2}^{-1}$ in either of the following ways:
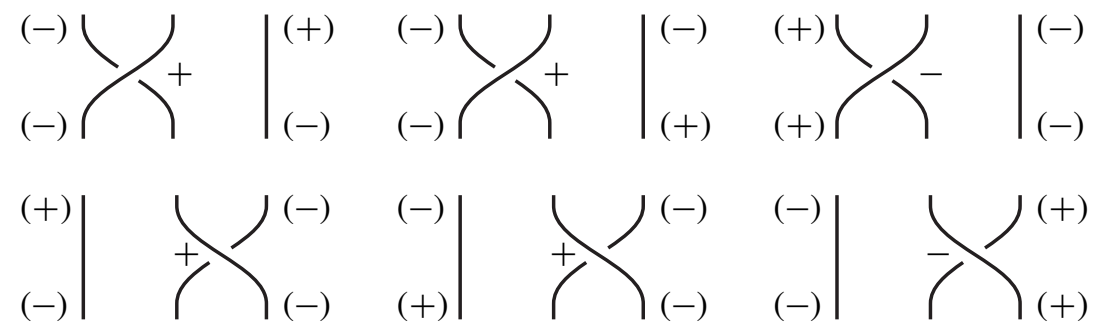

At the top of the two-bridge knot diagram, we give signs as in (1). When two copies of $\sigma_{1}$ (or $\sigma_{2}^{-1}$ ) appear successively, we give the same sign to their crossings, and choose either of (2) in such a way that the parenthesized signs coincide at the connection points. When $\sigma_{1}$ (resp. $\sigma_{2}^{-1}$ ) appears after $\sigma_{2}^{-1}$ (resp. $\sigma_{1}$ ), we choose either of (2) in such a way that the parenthesized signs coincide.

For an open two-bridge knot diagram with such signs, we give a recursive formula of $g_{i}$, as follows. When the $i^{\text {th }}$ copy of $\sigma_{1}$ or $\sigma_{2}^{-1}$ is labeled as in the following picture or its mirror image

we set

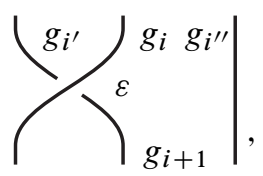

$$
g_{i+1}=v_{i} v_{i^{\prime \prime}} g_{i} g_{i^{\prime \prime}}+\varepsilon g_{i^{\prime}},
$$

where we have

$$
v_{j}= \begin{cases}u & \text { if the strand of } g_{j} \text { is a thick line } \\ 1 & \text { otherwise. }\end{cases}
$$

By (4), we redefine the values of $g_{i}$ recursively.

Lemma C.1 When the top of the $i^{\text {th }}$ copy of $\sigma_{1}$ or $\sigma_{2}^{-1}$ are labeled as in the following picture

$$
(\varepsilon)\left|g_{i^{\prime}} \quad\right| g_{i} g_{i^{\prime \prime}} \mid\left(\varepsilon^{\prime}\right)
$$

we have that

$$
\varepsilon v_{i^{\prime}} g_{i^{\prime}}^{2}+v_{i} g_{i}^{2}+\varepsilon^{\prime} v_{i^{\prime \prime}} g_{i^{\prime \prime}}^{2}=u g_{i^{\prime}} g_{i} g_{i^{\prime \prime}}
$$


Proof We show the lemma by induction on $i$. We show the case of $i+1$, assuming the case of $i$. The $i^{\text {th }}$ copy of $\sigma_{1}$ or $\sigma_{2}^{-1}$ is labeled as in the following picture or its mirror image:

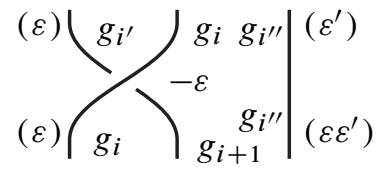

Then

$$
g_{i+1}=v_{i} v_{i^{\prime \prime}} g_{i} g_{i^{\prime \prime}}-\varepsilon g_{i^{\prime}}
$$

Hence,

$$
\begin{aligned}
\varepsilon v_{i} g_{i}^{2}+ & v_{i^{\prime}} g_{i+1}^{2}+\varepsilon \varepsilon^{\prime} v_{i^{\prime \prime}} g_{i^{\prime \prime}}^{2}-u g_{i} g_{i+1} g_{i^{\prime \prime}} \\
& =\varepsilon\left(\varepsilon v_{i^{\prime}} g_{i^{\prime}}^{2}+v_{i} g_{i}^{2}+\varepsilon^{\prime} v_{i^{\prime \prime}} g_{i^{\prime \prime}}^{2}-u g_{i^{\prime}} g_{i} g_{i^{\prime \prime}}\right) \\
& =0,
\end{aligned}
$$

noting that $v_{i^{\prime}} v_{i} v_{i^{\prime \prime}}=u$ by definition. Therefore, we obtain the lemma.

When the $i^{\text {th }}$ copy of $\sigma_{1}$ or $\sigma_{2}^{-1}$ is labeled as in (3), we set

$$
f_{i+1}^{\prime}=\varepsilon \cdot \frac{g_{i+1}}{g_{i^{\prime}}}
$$

Lemma C.2 We have $f_{i}^{\prime}=f_{i}$. That is, $g_{i}$ gives the numerator of $f_{i}$.

Proof We show the lemma by induction on $i$. To simplify the notation, we write the proof for $i=10$, assuming the case of $i<10$. We consider the $7^{\text {th }}, 8^{\text {th }}$ and $9^{\text {th }}$ copies of $\sigma_{1}$ or $\sigma_{2}^{-1}$. Since the recursive formula is invariant under the mirror image exchanging $\sigma_{1}$ and $\sigma_{2}^{-1}$, it is sufficient to consider the case where the $8^{\text {th }}$ copy is $\sigma_{1}$. Hence, there are the following 4 cases.

Case 1: $\boldsymbol{\sigma}_{\mathbf{1}}^{\mathbf{3}}$ The knot diagram of this part is labeled as in the following picture:

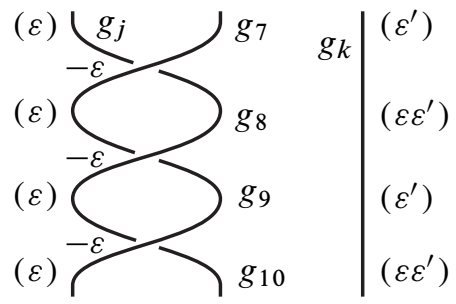

By the definition of $f_{i}^{\prime}$ and (40),

$$
f_{8}^{\prime}=-\varepsilon \cdot \frac{g_{8}}{g_{j}}, \quad f_{9}^{\prime}=-\varepsilon \cdot \frac{g_{9}}{g_{7}}, \quad f_{10}^{\prime}=-\varepsilon \cdot \frac{g_{10}}{g_{8}}, \quad f_{10}=f_{9}+1-\frac{f_{9}}{f_{8}} .
$$


Since $f_{8}^{\prime}=f_{8}$ and $f_{9}^{\prime}=f_{9}$, we can express $f_{10}-f_{10}^{\prime}$ using the $g_{*}$. Further, by (4),

$$
g_{8}=v_{9} v_{k} g_{7} g_{k}-\varepsilon g_{j}, \quad g_{9}=v_{8} v_{k} g_{8} g_{k}-\varepsilon g_{7}, \quad g_{10}=v_{9} v_{k} g_{9} g_{k}-\varepsilon g_{8},
$$

noting that $v_{7}=v_{9}$ by definition. By removing $g_{j}, g_{7}, g_{10}$ from the above mentioned expression of $f_{10}-f_{10}^{\prime}$, we can show by concrete calculation that

$$
f_{10}-f_{10}^{\prime}=1-\varepsilon^{2}=0 .
$$

Hence, the lemma holds for $i=10$ in this case.

Case 2: $\sigma_{1}^{\mathbf{2}} \sigma_{2}^{-1}$ The knot diagram of this part is labeled as in the following picture:

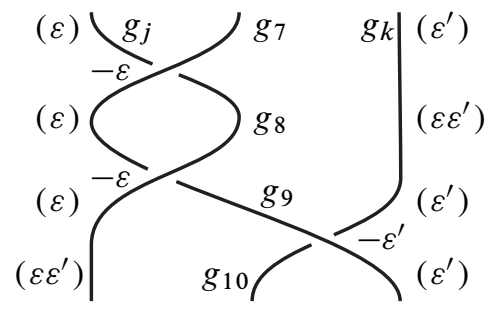

By the definition of $f_{i}^{\prime}$ and (40),

$$
f_{8}^{\prime}=-\varepsilon \cdot \frac{g_{8}}{g_{j}}, \quad f_{9}^{\prime}=-\varepsilon \cdot \frac{g_{9}}{g_{7}}, \quad f_{10}^{\prime}=-\varepsilon^{\prime} \cdot \frac{g_{10}}{g_{k}}, \quad f_{10}=f_{9}+\frac{\left(f_{9}-1\right)^{2}}{1-f_{9} / f_{8}} .
$$

Further, by (4),

$$
g_{8}=v_{9} v_{k} g_{7} g_{k}-\varepsilon g_{j}, \quad g_{9}=v_{8} v_{k} g_{8} g_{k}-\varepsilon g_{7}, \quad g_{10}=v_{8} v_{9} g_{8} g_{9}-\varepsilon^{\prime} g_{k} .
$$

Similarly as the above case, we can show by concrete calculation that

$$
\left(f_{10}-f_{10}^{\prime}\right)\left(1-\frac{f_{9}}{f_{8}}\right) g_{7}=\varepsilon^{\prime} v_{8} v_{9} g_{9}\left(\varepsilon v_{8} g_{8}^{2}+v_{9} g_{9}^{2}+\varepsilon^{\prime} v_{k} g_{k}^{2}-u g_{8} g_{9} g_{k}\right)=0,
$$

where we obtain the last equality by Lemma C.1. Hence, the lemma holds for $i=10$ in this case.

Case 3: $\sigma_{2}^{-1} \sigma_{1}^{2}$ The knot diagram of this part is labeled as in the following picture:

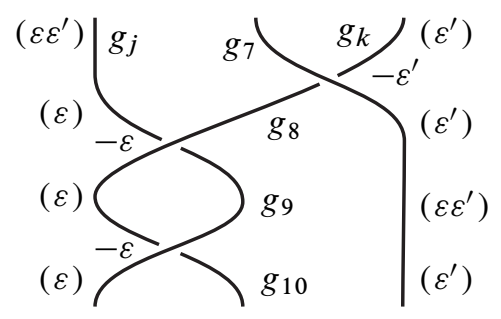


By the definition of $f_{i}^{\prime}$ and (40),

$$
f_{8}^{\prime}=-\varepsilon^{\prime} \cdot \frac{g_{8}}{g_{k}}, \quad f_{9}^{\prime}=-\varepsilon \cdot \frac{g_{9}}{g_{j}}, \quad f_{10}^{\prime}=-\varepsilon \cdot \frac{g_{10}}{g_{8}}, \quad f_{10}=f_{9}+1-\frac{f_{9}}{f_{8}} .
$$

Further, by (4),

$$
g_{8}=v_{9} v_{7} g_{j} g_{7}-\varepsilon^{\prime} g_{k}, \quad g_{9}=v_{7} v_{8} g_{7} g_{8}-\varepsilon g_{j}, \quad g_{10}=v_{7} v_{9} g_{7} g_{9}-\varepsilon g_{8} .
$$

Similar to Case 1, we can show by concrete calculation that

$$
f_{10}-f_{10}^{\prime}=1-\varepsilon^{2}=0 .
$$

Hence, the lemma holds for $i=10$ in this case.

Case 4: $\sigma_{2}^{-1} \sigma_{1} \sigma_{2}^{-1}$ The knot diagram of this part is labeled as in the following picture:

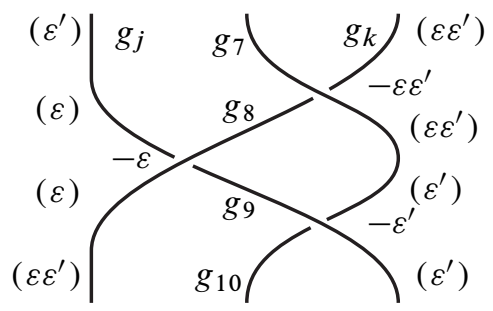

By the definition of $f_{i}^{\prime}$ and (40),

$$
f_{8}^{\prime}=-\varepsilon \varepsilon^{\prime} \cdot \frac{g_{8}}{g_{k}}, \quad f_{9}^{\prime}=-\varepsilon \cdot \frac{g_{9}}{g_{j}}, \quad f_{10}^{\prime}=-\varepsilon^{\prime} \cdot \frac{g_{10}}{g_{7}}, \quad f_{10}=f_{9}+\frac{\left(f_{9}-1\right)^{2}}{1-f_{9} / f_{8}} .
$$

Further, by (4),

$$
g_{8}=v_{9} v_{7} g_{j} g_{7}-\varepsilon \varepsilon^{\prime} g_{k}, \quad g_{9}=v_{7} v_{8} g_{7} g_{8}-\varepsilon g_{j}, \quad g_{10}=v_{8} v_{9} g_{8} g_{9}-\varepsilon^{\prime} g_{7} .
$$

Similar to Case 2, we can show by concrete calculation that

$$
\left(f_{10}-f_{10}^{\prime}\right)\left(1-\frac{f_{9}}{f_{8}}\right) g_{j}=\varepsilon^{\prime} u g_{9}\left(\varepsilon v_{8} g_{8}^{2}+v_{9} g_{9}^{2}+\varepsilon^{\prime} v_{7} g_{7}^{2}-u g_{8} g_{9} g_{7}\right)=0 \text {. }
$$

Hence, the lemma holds for $i=10$ in this case, as required.

\section{Appendix D: Representations of two-bridge knot groups}

It is known [22] that parabolic representations of a two-bridge knot group are characterized by roots of some polynomial equation. In this appendix, we describe this polynomial equation in terms of 3-braids. An aim of this appendix is to show that 
the equation $f_{m}(x)=0$ has no repeated root (Lemma D.8), which means the nondegeneracy of the Hesse matrix discussed in Section 4.3. Further, we see that we can apply Theorem 1.1 to most roots of the equation $f_{m}(x)=0$ for the $(\alpha, \beta)$ two-bridge knot with any $\alpha<50$ in Remark D.12, and to the root corresponding to the complete hyperbolic structure of the knot complement in Remark D.14.

We briefly review the space of conjugacy classes of $\mathrm{SL}_{2} \mathbb{C}$ representations of a twobridge knot group; for details, see Riley [21;22]. Let $\alpha$ and $\beta$ be coprime integers such that $0<\beta<\alpha$ and $\alpha$ is odd. It is known that the $(\alpha, \beta)$ two-bridge knot group is presented by

$$
\langle a, b \mid w a=b w\rangle,
$$

where we set $w=a^{\varepsilon_{1}} b^{\varepsilon_{1}} \cdots a^{\varepsilon_{\alpha-2}} b^{\varepsilon_{\alpha-1}}$ and $\varepsilon_{i}=(-1)^{\lfloor i \beta / \alpha\rfloor}$. Hence, the space of conjugacy classes of $\mathrm{SL}_{2} \mathbb{C}$ representations of the $(\alpha, \beta)$ two-bridge knot group can be identified with

$$
\left\{(A, B) \in \mathrm{SL}_{2} \mathbb{C} \times \mathrm{SL}_{2} \mathbb{C} \mid W A=B W\right\} / \text { conjugation }
$$

where we set $W=A^{\varepsilon_{1}} B^{\varepsilon_{1}} \cdots A^{\varepsilon_{\alpha-2}} B^{\varepsilon_{\alpha-1}}$. It is known [21; 22] that, by letting

$$
t=\operatorname{trace} A=\operatorname{trace} B, \quad u=\operatorname{trace} A B-2,
$$

we have that

$$
W A-B W=\mathcal{P}_{\alpha, \beta}\left(t^{2}, u\right)(A-B)
$$

with some polynomial $\mathcal{P}_{\alpha, \beta}\left(t^{2}, u\right)$ in $t^{2}$ and $u$. Hence, the space (1) can be identified with

$$
\left\{(t, u) \in \mathbb{C}^{2} \mid \mathcal{P}_{\alpha, \beta}\left(t^{2}, u\right)=0\right\} .
$$

In particular, parabolic representations are given by roots of the equation $\mathcal{P}_{\alpha, \beta}(4, u)=0$. It is known [21] that $\mathcal{P}_{\alpha, \beta}(4, u)$ is a polynomial in $u$ of degree $(\alpha-1) / 2$, and the equation $\mathcal{P}_{\alpha, \beta}(4, u)=0$ has $(\alpha-1) / 2$ distinct roots. Hence, $\left(\partial \mathcal{P}_{\alpha, \beta} / \partial u\right)\left(4, u_{0}\right) \neq 0$ for each root $u_{0}$, and the tangent space of (3) at $\left(4, u_{0}\right)$ is 1 -dimensional, which implies the following lemma.

Lemma D.1 At each parabolic representation, $H^{1}\left(C^{*}\right)$ is 1-dimensional.

Proof The cohomology $H^{1}\left(C^{*}\right)$ is isomorphic to the Zariski tangent space of (3) at $\left(4, u_{0}\right)$, where $u_{0}$ is a root of $\mathcal{P}_{\alpha, \beta}(4, u)=0$. Since $\left(\partial \mathcal{P}_{\alpha, \beta} / \partial u\right)\left(4, u_{0}\right) \neq 0$ as mentioned above, the total differential of $\mathcal{P}_{\alpha, \beta}$ does not vanish at $\left(4, u_{0}\right)$. Hence, the Zariski tangent space at $\left(4, u_{0}\right)$ is 1 -dimensional. 
We consider to reconstruct $\mathcal{P}_{\alpha, \beta}(4, u)$ in terms of 3 -braids, while the above construction of $\mathcal{P}_{\alpha, \beta}(4, u)$ is obtained from the normal form of the two-bridge knot. In our notation, the parameter $u$ is presented by

$$
\begin{aligned}
u=\operatorname{trace}\left(W_{0}^{ \pm 1} \cdot X_{0} X_{0}^{\prime}\right)-2 & =\operatorname{trace}\left(\begin{array}{cc}
0 & 1 \\
-1 & 2
\end{array}\right)^{ \pm 1}\left(\begin{array}{cc}
1 & x-1 \\
0 & 1
\end{array}\right)-2 \\
& = \pm(x-1),
\end{aligned}
$$

where we choose the sign in such a way that $W_{0}^{ \pm 1}$ and $X_{0} X_{0}^{\prime}$ correspond to conjugate meridians, which depends on the orientations of strands in the top part of an open two-bridge knot. We consider the continued fraction expansion of $\alpha / \beta$,

$$
\frac{\alpha}{\beta}=n_{1}+\frac{1}{n_{2}+\ddots_{+\frac{1}{n_{\ell}}}}=\left[n_{1}, n_{2}, \ldots, n_{\ell}\right],
$$

where we use the notation of the right-hand side to express this continued fraction expansion. In the following of this appendix (and in Appendix C), we choose the sign $u=x-1$. (When $u=-(x-1)$, the sign of $u$ is changed in the following argument.) It is known, see eg Burde and Zieschang [2], that the $(\alpha, \beta)$ two-bridge knot can be presented by the plat closure of $\sigma_{1}^{n_{1}} \sigma_{2}^{-n_{2}} \cdots \sigma_{1}^{n_{\ell}} j$ if $\ell$ is odd, and $\sigma_{1}^{n_{1}} \sigma_{2}^{-n_{2}} \cdots \sigma_{2}^{-n_{\ell}}$ if $\ell$ is even. For example, for $17 / 5=[3,2,2]$, the $(17,5)$ two-bridge knot can be presented by the following form:

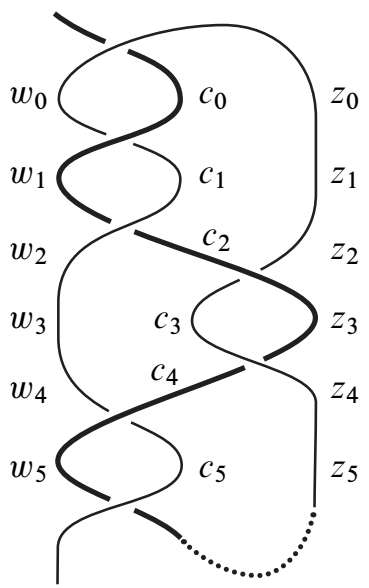

We associate parameters $w_{i}, c_{i}, z_{i}$ to strands of the knot diagram as shown above; we explain their meanings below. We recall that [22] that $\mathcal{P}_{\alpha, \beta}(4, u)$ is obtained by considering the representation $\rho$ of the two-bridge knot group into $\mathrm{SL}_{2} \mathbb{C}$ determined 
by the following two generators:

$$
A=\left(\begin{array}{ll}
1 & 1 \\
0 & 1
\end{array}\right) \underbrace{B=\left(\begin{array}{ll}
1 & 0 \\
u & 1
\end{array}\right)}
$$

We note that $u \neq 0$, since $A$ and $B$ are conjugate. We define the values of $w_{i}, c_{i}, z_{i}$ and $c_{i}^{\prime}$ as polynomials in $u$ by

$$
\begin{aligned}
& w_{i}=\operatorname{trace} \rho\left(\mid \frac{d-2, \quad c_{i}=\operatorname{trace} \rho(-1-1}{\mathrm{l}}-2\right. \text {, } \\
& z_{i}=\operatorname{trace} \rho\left(\frac{1}{1} \mid\right)-2, \quad c_{i}^{\prime}=\operatorname{trace} \rho\left(\begin{array}{ll}
1 & 1 \\
1 & 1
\end{array}\right)-2 \text {, }
\end{aligned}
$$

where each loop is at the height of each parameters. We note that the trace of $\rho$ of a loop does not depend on the base point and the orientation of the loop, since $\rho$ is a $\mathrm{SL}_{2} \mathbb{C}$ representation.

Lemma D.2 For each $i$ we have

$$
c_{i}+c_{i}^{\prime}=4-\left(w_{i}+2\right)\left(z_{i}+2\right) .
$$

Proof We consider the following 3 elements of the fundamental group at the height of $c_{i}$, and define their images by $\rho$ to be $P, Q$ and $R$ :

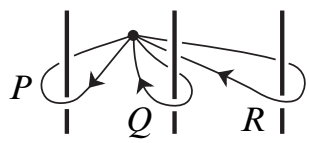

Then from the definitions of $w_{i}, c_{i}, z_{i}, c_{i}^{\prime}$, we have that

$$
\text { trace } P Q=z_{i}+2, \quad \text { trace } Q R=w_{i}+2, \quad \text { trace } P R=c_{i}+2,
$$$$
\text { trace } P Q R Q^{-1}=c_{i}^{\prime}+2, \quad \operatorname{trace} P Q R=\operatorname{trace}\left(\begin{array}{ll}
1 & 1 \\
0 & 1
\end{array}\right)=2 \text {. }
$$

Since $Q$ is a parabolic matrix in $\mathrm{SL}_{2} \mathbb{C}$, we have that $Q^{2}-2 Q+E=0$, where $E$ denotes the identity matrix. Hence,

$$
c_{i}^{\prime}+2=\operatorname{trace} P Q R(2 E-Q)=4-\operatorname{trace} P Q R Q .
$$


Further, since $(Q R)^{2}-\left(w_{i}+2\right) Q R+E=0$, we have that $Q R Q=\left(w_{i}+2\right) Q-R^{-1}=$ $\left(w_{i}+2\right) Q+R-2 E$. Therefore,

$$
c_{i}^{\prime}+2=4-\operatorname{trace} P\left(\left(w_{i}+2\right) Q+R-2 E\right)=4-\left(w_{i}+2\right)\left(z_{i}+2\right)-\left(c_{i}+2\right)+4 .
$$

Hence, we obtain the required formula of the lemma.

Lemma D.3 The values of $w_{i}, c_{i}, z_{i}$ are determined by $\left(w_{0}, c_{0}, z_{0}\right)=\left(-u, u^{2}, u\right)$ and the recursive formula

$$
\begin{aligned}
&\left(w_{i+1}, c_{i+1}, z_{i+1}\right) \\
& \quad= \begin{cases}\left(c_{i}, 4-w_{i}-\left(c_{i}+2\right)\left(z_{i}+2\right), z_{i}\right) & \text { if } \sigma_{1} \text { is between } c_{i} \text { and } c_{i+1}, \\
\left(w_{i}, 4-z_{i}-\left(c_{i}+2\right)\left(w_{i}+2\right), c_{i}\right) & \text { if } \sigma_{2}^{-1} \text { is between } c_{i} \text { and } c_{i+1} .\end{cases}
\end{aligned}
$$

Proof We show the recursive formula in the case where $\sigma_{1}$ is between $c_{i}$ and $c_{i+1}$. In this case, the defining loops of the parameters are related, as follows:
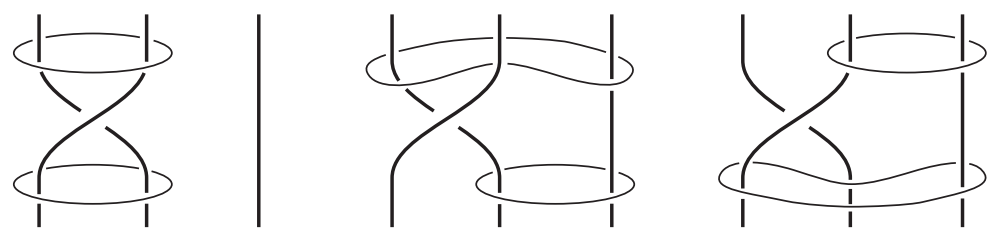

Hence,

$$
z_{i+1}=z_{i}, \quad w_{i+1}=c_{i}, \quad c_{i+1}^{\prime}=w_{i} .
$$

Therefore, we obtain the required recursive formula by Lemma D.2.

In the case where $\sigma_{2}^{-1}$ is between $c_{i}$ and $c_{i+1}$, we can show the required recursive formula in a similar way as above.

Lemma D.4 We assume that the strands are labeled as below in the sense of Lemma C.1 at the height of $w_{i}, c_{i}, z_{i}$ :

$$
(\varepsilon)\left|g_{i^{\prime}} \quad\right| g_{i} g_{i^{\prime \prime}} \mid\left(\varepsilon^{\prime}\right)
$$

Then

$$
w_{i}=-\varepsilon^{\prime} v_{i^{\prime}} u g_{i^{\prime}}^{2}, \quad c_{i}=-\varepsilon \varepsilon^{\prime} v_{i} u g_{i}^{2}, \quad z_{i}=-\varepsilon v_{i^{\prime \prime}} u g_{i^{\prime \prime}}^{2} .
$$

Proof We show the lemma by induction on $i$, proving that the statement holds for $i+1$ assuming that it does for $i$. 
When there is $\sigma_{1}$ between $g_{i}$ and $g_{i+1}$, this part is labeled as in the following form:

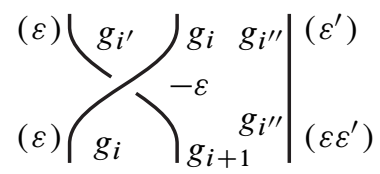

Since $w_{i+1}=c_{i}$ and $z_{i+1}=z_{i}$ by Lemma D.3, we obtain the required formulas for $w_{i+1}$ and $z_{i+1}$. Further, since $c_{i+1}=4-w_{i}-\left(c_{i}+2\right)\left(z_{i}+2\right)$ by Lemma D.3, we have that

$$
\begin{gathered}
c_{i+1}+\varepsilon^{\prime} v_{i^{\prime}} u g_{i+1}^{2}=4+\varepsilon^{\prime} v_{i^{\prime}} u g_{i^{\prime}}^{2}-\left(-\varepsilon \varepsilon^{\prime} v_{i} u g_{i}^{2}+2\right)\left(-\varepsilon v_{i^{\prime \prime}} u g_{i^{\prime \prime}}^{2}+2\right) \\
+\varepsilon^{\prime} v_{i^{\prime}} u\left(v_{i} v_{i^{\prime \prime}} g_{i} g_{i^{\prime \prime}}-\varepsilon g_{i^{\prime}}\right)^{2} \\
=2 \varepsilon \varepsilon^{\prime} u\left(\varepsilon v_{i^{\prime}} g_{i^{\prime}}^{2}+v_{i} g_{i}^{2}+\varepsilon^{\prime} v_{i^{\prime \prime}} g_{i^{\prime \prime}}^{2}-u g_{i^{\prime}} g_{i} g_{i^{\prime \prime}}\right)=0,
\end{gathered}
$$

where we obtain the last equality by Lemma C.1. Hence, we obtain the required formula for $c_{i+1}$, as required.

When there is $\sigma_{2}^{-1}$ between $g_{i}$ and $g_{i+1}$, we can obtain the required formulas in a similar way as above.

We consider the degrees of $w_{i}, c_{i}, z_{i}$ as polynomials in $u$. They are shown for the example (5), as follows:

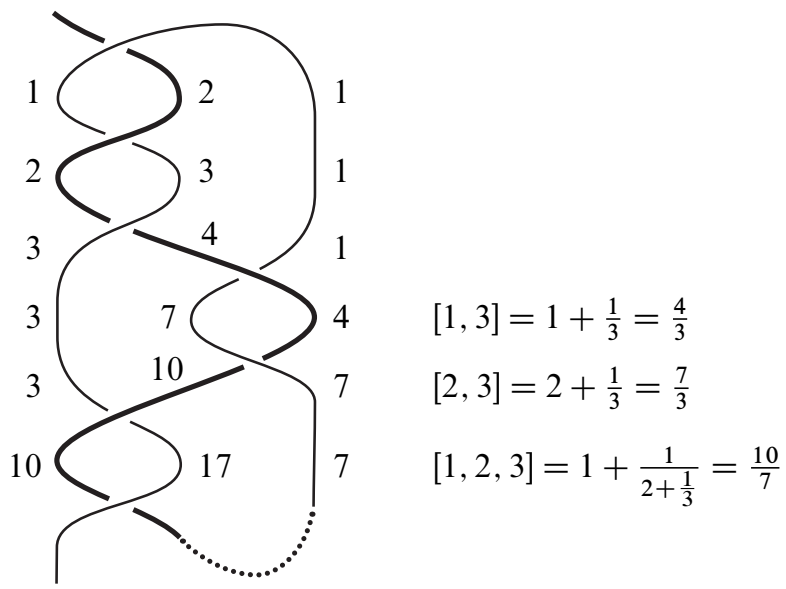

In this picture, we can observe that the degrees of $w_{i}$ and $z_{i}$ can be presented in terms of the numerator and the denominator of the continued fraction expansion corresponding to the 3-braid from the top to the height of $w_{i}$ and $z_{i}$. This observation can be justified for a general case in the following lemma. 
Lemma D.5 We assume that the 3-braid from the top to the height of $w_{i}$ and $z_{i}$ is presented by $\sigma_{1}^{n_{1}} \sigma_{2}^{-n_{2}} \cdots \sigma_{1}^{n_{\ell}}$ if $\ell$ is odd, and $\sigma_{1}^{n_{1}} \sigma_{2}^{-n_{2}} \cdots \sigma_{2}^{-n_{\ell}}$ if $\ell$ is even. Then

$$
\left[n_{\ell}, n_{\ell-1}, \ldots, n_{2}, n_{1}\right]= \begin{cases}\frac{\operatorname{deg} w_{i}}{\operatorname{deg} z_{i}} & \text { if } \ell \text { is odd, } \\ \frac{\operatorname{deg} z_{i}}{\operatorname{deg} w_{i}} & \text { if } \ell \text { is even. }\end{cases}
$$

Proof We note that

$$
\operatorname{deg} c_{i}=\operatorname{deg} w_{i}+\operatorname{deg} z_{i},
$$

which can be shown by induction on $i$ by Lemma D.3. We show the lemma by induction on $n_{1}+n_{2}+\cdots+n_{\ell}$. In the following of this proof, we show the lemma for $\sigma_{1}^{n_{1}} \sigma_{2}^{-n_{2}} \cdots \sigma_{1}^{n_{\ell}} \cdot \sigma_{1}$ and $\sigma_{1}^{n_{1}} \sigma_{2}^{-n_{2}} \cdots \sigma_{1}^{n_{\ell}} \cdot \sigma_{2}^{-1}$, assuming the case of $\sigma_{1}^{n_{1}} \sigma_{2}^{-n_{2}} \cdots \sigma_{1}^{n_{\ell}}$, when $\ell$ is odd. (When $\ell$ is even, we can show this claim similarly.)

For $\sigma_{1}^{n_{1}} \sigma_{2}^{-n_{2}} \cdots \sigma_{1}^{n_{\ell}} \cdot \sigma_{1}$, the continued fraction expansion is given by

$$
\begin{aligned}
{\left[n_{\ell}+1, n_{\ell-1}, \ldots, n_{2}, n_{1}\right] } & =1+\left[n_{\ell}, n_{\ell-1}, \ldots, n_{2}, n_{1}\right] \\
& =1+\frac{\operatorname{deg} w_{i}}{\operatorname{deg} z_{i}}=\frac{\operatorname{deg} w_{i}+\operatorname{deg} z_{i}}{\operatorname{deg} z_{i}}=\frac{\operatorname{deg} w_{i+1}}{\operatorname{deg} z_{i+1}},
\end{aligned}
$$

where we obtain the last equality by (7) and Lemma D.3. Hence, the lemma holds for $\sigma_{1}^{n_{1}} \sigma_{2}^{-n_{2}} \cdots \sigma_{1}^{n_{\ell}} \cdot \sigma_{1}$.

For $\sigma_{1}^{n_{1}} \sigma_{2}^{-n_{2}} \cdots \sigma_{1}^{n_{\ell}} \cdot \sigma_{2}^{-1}$, the continued fraction expansion is given by

$$
\begin{aligned}
{\left[1, n_{\ell}, \ldots, n_{2}, n_{1}\right] } & =1+\frac{1}{\left[n_{\ell}, \ldots, n_{2}, n_{1}\right]} \\
& =1+\frac{\operatorname{deg} z_{i}}{\operatorname{deg} w_{i}}=\frac{\operatorname{deg} w_{i}+\operatorname{deg} z_{i}}{\operatorname{deg} w_{i}}=\frac{\operatorname{deg} z_{i+1}}{\operatorname{deg} w_{i+1}},
\end{aligned}
$$

where we obtain the last equality by (7) and Lemma D.3. Hence, the lemma holds for $\sigma_{1}^{n_{1}} \sigma_{2}^{-n_{2}} \cdots \sigma_{1}^{n_{\ell}} \cdot \sigma_{2}^{-1}$, as required.

As in the notation of Section 4.3 and Appendix C, we consider an open two-bridge knot diagram whose lowest parameter is $g_{m}$.

Lemma D.6 For the $(\alpha, \beta)$ two-bridge knot, $g_{m}$ is of degree $\frac{\alpha-1}{2}$.

Proof Let $\left[n_{1}, n_{2}, \ldots, n_{\ell}\right]$ be the continued fraction expansion corresponding to the 3 -braid from the top to the height of $g_{m}$. Then the $(\alpha, \beta)$ two-bridge knot is given by the plat closure of the 3 -braid corresponding to $\left[n_{1}, n_{2}, \ldots, n_{\ell-1}, n_{\ell}+1\right]=\frac{\alpha}{\beta}$. Since 
the continued fraction expansion of the converse order gives an equivalent two-bridge knot,

$$
\frac{\alpha}{\beta^{\prime}}=\left[n_{\ell}+1, n_{\ell-1}, \ldots, n_{2}, n_{1}\right]=1+\left[n_{\ell}, n_{\ell-1}, \ldots, n_{2}, n_{1}\right],
$$

for some $\beta^{\prime}$ (see [2]). By Lemma D.5, its numerator is given by

$$
\alpha=\operatorname{deg} w_{m}+\operatorname{deg} z_{m}=\operatorname{deg} c_{m}=2 \operatorname{deg} g_{m}+1,
$$

where we obtain the second equality by (7), and obtain the third equality by Lemma D.4. Hence, we obtain the lemma.

Lemma D.7 The parameter $g_{m}$ is equal to a nonzero scalar multiple of $\mathcal{P}_{\alpha, \beta}(4, \varepsilon u)$, where we choose the sign $\varepsilon= \pm 1$ as in (4). In particular, the equation $g_{m}=0$ has no repeated root.

Proof Let $u$ be a root of $\mathcal{P}_{\alpha, \beta}(4, \varepsilon u)$. Then we have a parabolic representation of the knot group determined by (6). It follows from the definition of $c_{m}$ that $c_{m}=0$. Hence, by Lemma D.4, $g_{m}=0$.

We recall [21] that $\mathcal{P}_{\alpha, \beta}(4, u)$ is a polynomial in $u$ of degree $(\alpha-1) / 2$, and the equation $\mathcal{P}_{\alpha, \beta}(4, u)=0$ has $(\alpha-1) / 2$ distinct roots. Since $g_{m}$ is of degree $(\alpha-1) / 2$ by Lemma D.6, we obtain the lemma.

We recall that in Section 4.3, we regarded $x_{m}$ as a rational function of $x$ and defined it to be $f_{m}(x)$.

Lemma D.8 The equation $f_{m}(x)=0$ has no repeated root.

Proof By Lemma C.2, $g_{m}$ gives the numerator of $f_{m}$. Since the equation $g_{m}=0$ has no repeated root by Lemma D.7, we obtain the lemma.

This lemma suggests a positive answer to [24, Conjecture II.5.10(1)].

Lemma D.9 The equation $f_{m}(x)=0$ does not have a root $x=1$.

Proof Since $u \neq 0$ as mentioned at (6), the equation $\mathcal{P}_{\alpha, \beta}(4, \varepsilon u)=0$ does not have a root $u=0$. Hence, by Lemma D.7, the equation $g_{m}=0$ does not have a root $u=0$. Since $g_{m}$ gives the numerator of $f_{m}$, the equation $f_{m}=0$ does not have a root $x=1$, noting that $u= \pm(x-1)$. 
Example D.10 For the $(7,3)$ two-bridge knot, we compare $\mathcal{P}_{\alpha, \beta}(4, u)=0$ and $f_{3}(x)=0$.

In this case, $W=A B A^{-1} B^{-1} A B$. Further, by (2), we have $A^{2}-t A+E=0$, $B^{2}-t B+E=0,(A B)^{2}-(u+2) A B+E=0$, where $E$ denotes the identity matrix. Hence, by calculating $W A-B W$ concretely, we have that

$$
\mathcal{P}_{\alpha, \beta}\left(t^{2}, u\right)=u^{3}+\left(5-t^{2}\right) u^{2}+\left(6-t^{2}\right) u+1 .
$$

Therefore, parabolic representations are given by roots of the equation

$$
\mathcal{P}_{\alpha, \beta}(4, u)=u^{3}+u^{2}+2 u+1=0 .
$$

On the other hand, since $\frac{7}{3}=2+\frac{1}{3}$, this two-bridge knot is isotopic to the plat closure of $\sigma_{1}^{2} \sigma_{2}^{-3}$. Hence, by (40), the hyperbolicity equations can be rewritten as

$$
x_{1}=x, \quad x_{2}=x^{2}-x+1, \quad x_{3}=f_{3}(x)=\frac{x^{3}-2 x^{2}+3 x-1}{x}=0 .
$$

By setting $x=u+1$, we can verify that the last equation is equivalent to the above mentioned equation.

Example D.11 For $45 / 7=[6,2,3]$, we consider the $(45,7)$ two-bridge knot, which is given by the plat closure of $\sigma_{1}^{6} \sigma_{2}^{-2} \sigma_{1}^{3}$. We can obtain by concrete calculation that

$$
f_{9}=\frac{x\left(x^{21}+9 x^{20}+21 x^{19}-34 x^{18}-183 x^{17}+\cdots-102 x^{4}-48 x^{3}-4 x^{2}+8 x+1\right)}{x^{9}+4 x^{8}-13 x^{6}-3 x^{5}+15 x^{4}+2 x^{3}-x^{2}-3 x-1} \text {. }
$$

The equation $f_{9}(x)=0$ has the root $x=0$ and the other 21 roots.

When $x=0$, we can show by concrete calculation that $x_{0}=x_{3}=x_{8}=\infty, x_{1}=x_{4}=$ $x_{9}=0$ and $x_{2}=x_{5}=x_{6}=x_{7}=1$. In this case, we can not apply Theorem 1.1, since there exist hyperbolicity parameters 0 and $\infty$ except for the first and the last parameters. As we see in Remark D.12 below, the corresponding parabolic representation is not faithful in this case.

For each of the other 21 roots, it is a root of an irreducible polynomial of degree 21 . As shown in Appendix C, $x_{i}(0<i<9)$ can be presented by a rational function $f_{i}$ of $x$ whose numerator and denominator are polynomial of degree less than 21 . Hence, $x_{i} \in \mathbb{C}-\{0\}$ for $0<i<9$. Therefore, we can apply Theorem 1.1 for each of these 21 roots.

Remark D.12 As we see in Example D.11, there might exist some exceptional cases where we can not apply Theorem 1.1. We explain when such exceptional cases appear for the $(\alpha, \beta)$ two-bridge knot with $\alpha<50$ in this remark. 
As observed by Riley in [23], for many two-bridge knots, $\mathcal{P}_{\alpha, \beta}(4, u)$ is an irreducible polynomial in $u$. In this case, we can show similarly as in Example D.11 that we can apply Theorem 1.1 to each root of the equation $f_{m}(x)=0$.

There exist some exceptional cases where $\mathcal{P}_{\alpha, \beta}(4, u)$ is reducible. A typical case is a case where the two-bridge knot group has an epimorphism onto another smaller two-bridge knot group (see the first author, Riley and Sakuma [19] for details); in such a case, the $\mathrm{SL}_{2} \mathbb{C}$ representation space has an irreducible component consisting of representations which factor through the smaller two-bridge knot group. By computer search, we can show which two-bridge knot has reducible $\mathcal{P}_{\alpha, \beta}(4, u)$, and check whether $x_{i} \in \mathbb{C}-\{0\}$ for the roots of the hyperbolicity equations for such a two-bridge knot. We recall, see eg [2], that the $(\alpha, \beta)$ two-bridge knot is equivalent (allowing the mirror image) to the $\left(\alpha^{\prime}, \beta^{\prime}\right)$ two-bridge knot if $\alpha=\alpha^{\prime}$ and $\beta^{\prime}= \pm \beta^{ \pm 1}$ in $\mathbb{Z} / \alpha \mathbb{Z}$ (for any choice of signs). We can show by concrete calculation that, for the $(\alpha, \beta)$ two-bridge knot with $\alpha<50$, there exists such a "bad" root only if $\frac{\alpha}{\beta}$ is equivalent (up to the above equivalence) to one of

$$
\begin{gathered}
\frac{27}{5}=[5,2,2]=[6,-2,3], \quad \frac{33}{5}=[6,1,1,2]=[6,2,-3], \\
\frac{39}{7}=[5,1,1,3]=[6,-2,-3], \quad \frac{45}{7}=[6,2,3], \\
\frac{45}{19}=[2,2,1,2,2]=[3,-2,3,-2,3] .
\end{gathered}
$$

As shown in [19], these two-bridge knot groups have epimorphisms onto the knot group of the trefoil knot (the $(3,1)$ two-bridge knot). We can show by concrete calculation similarly as in Example D.11 that the above two-bridge knot has one "bad" root $x=0$ and the other "good" $(\alpha-3) / 2$ roots. Hence, we can apply Theorem 1.1 to most cases for the $(\alpha, \beta)$ two-bridge knot with $\alpha<50$.

Remark D.13 For a "bad" root of the above remark, there exists a continuous map $\eta$ from the complement of the $(\alpha, \beta)$ two-bridge knot $K$ to the complement of a smaller two-bridge knot $K^{\prime}$; see [19]. The parabolic representation corresponding to this root can be induced from a parabolic representation of the knot group of $K^{\prime}$ by pulling back by $\eta$. Hence, the cochain complex of $S^{3}-K$ with the $\mathfrak{s l}_{2} \mathbb{C}$ coefficient twisted by this parabolic representation can be obtained from a cochain complex of $S^{3}-K^{\prime}$ by pulling back by $\eta$.

Remark D.14 In this remark, we explain that we can apply Theorem 1.1 to the root corresponding to the holonomy representation of the complete hyperbolic structure ${ }^{9}$ of the knot complement. We assume that there existed $x_{i}=0$ or $\infty$ for some $i$ with

\footnotetext{
${ }^{9}$ It is known that a two-bridge knot is hyperbolic unless it is the $(2, n)$ torus knot.
} 
$0<i<m$. Then by the construction of a parabolic representation explained in this appendix, we can show that the corresponding parabolic representation is not faithful at the height of such $x_{i}$. Since the holonomy representation of the complete hyperbolic structure is faithful, this is a contradiction. Therefore, $x_{i} \in \mathbb{C}-\{0\}$ for any $i$ with $0<i<m$, and we can apply Theorem 1.1 to the root corresponding to the complete hyperbolic structure.

\section{Appendix E: The case of nonhyperbolic two-bridge knots}

It is known that a nonhyperbolic two-bridge knot is a $(2, n)$ torus knot. In this appendix, we explain about roots of the hyperbolicity equation and representations in the case of the $(2, n)$ torus knot. We note that $n$ is odd.

As a two-bridge knot, the $(2, n)$ torus knot is obtained as a plat closure of $\sigma_{1}^{n}$, whose diagram is parameterized by hyperbolicity parameters $x_{0}, x_{1}, \ldots, x_{m}$, setting $m=n-2$. As mentioned in Appendix $\mathrm{C}$, we consider rational functions $f_{i}(x)$, which is presented by $f_{i}=g_{i} / g_{i-2}$, where the $g_{i}$ are given by $g_{1}=x, g_{2}=x+1$ and

$$
g_{i+1}= \begin{cases}g_{i}+g_{i-1} & \text { if } i \text { is odd, } \\ (x-1) g_{i}+g_{i-1} & \text { if } i \text { is even. }\end{cases}
$$

Hence, setting $x=\zeta-1+\zeta^{-1}$, we can verify the following formula by concrete calculation,

$$
g_{i}= \begin{cases}\frac{\zeta^{i+2}+1}{\zeta^{(i+1) / 2}(\zeta+1)} & \text { if } i \text { is odd } \\ \frac{\zeta^{i+2}-1}{\zeta^{i / 2}(\zeta+1)(\zeta-1)} & \text { if } i \text { is even. }\end{cases}
$$

Therefore, noting that $m$ is odd, the roots of $f_{m}(x)=0$ are given by

$$
x=2 \cos \left(\frac{k \pi}{n}\right)-1 \quad \text { for } k=1,3,5, \ldots, n-2 .
$$

We can verify by concrete calculation that, if $n$ and $k$ are coprime, the roots (1) are "good" roots in the sense of Remark D.12, and we can apply Theorem 1.1 to them.

We comment on the representation of the $(2, n)$ torus knot group induced from the holonomy representation of the 2-dimensional hyperbolic structure of the basis of Seifert fibration of the $(2, n)$ torus knot complement. The basis of this Seifert fibration is a punctured 2-sphere with two cone singularities whose cone angles are $\pi$ and $2 \pi / n$. We consider the hyperbolic structure of this 2-orbifold such that the puncture is a cusp; this hyperbolic structure is obtained by gluing two copies of a hyperbolic triangle whose angles are $\pi / 2, \pi / n$ and 0 . The holonomy representation of this hyperbolic 
structure induces a $\mathrm{PSL}_{2} \mathbb{R}$ representation of the $(2, n)$ torus knot group. We can show by concrete calculation that the root (1) of $k=n-2$ gives this representation. Since $n$ and $n-2$ are coprime, we can apply Theorem 1.1 to this root.

\section{References}

[1] J E Andersen, S K Hansen, Asymptotics of the quantum invariants for surgeries on the figure 8 knot, J. Knot Theory Ramifications 15 (2006) 479-548 MR2221531

[2] G Burde, H Zieschang, Knots, de Gruyter Studies in Math. 5, de Gruyter, Berlin (1985) MR808776

[3] T Dimofte, S Garoufalidis, The quantum content of the gluing equations, Geom. Topol. 17 (2013) 1253-1315 MR3073925

[4] T Dimofte, S Gukov, J Lenells, D Zagier, Exact results for perturbative ChernSimons theory with complex gauge group, Commun. Number Theory Phys. 3 (2009) 363-443 MR2551896

[5] J Dubois, Computational aspects in Reidemeister torsion and Chern-Simons theories, from: "Chern-Simons gauge theory: 20 years after", (J E Andersen, H U Boden, A Hahn, B Himpel, editors), AMS/IP Stud. Adv. Math. 50, Amer. Math. Soc. (2011) 43-64 MR2809446

[6] J Dubois, V Huynh, Y Yamaguchi, Nonabelian Reidemeister torsion for twist knots, J. Knot Theory Ramifications 18 (2009) 303-341 MR2514847

[7] L D Faddeev, Discrete Heisenberg-Weyl group and modular group, Lett. Math. Phys. 34 (1995) 249-254 MR1345554

[8] L D Faddeev, R M Kashaev, A Y Volkov, Strongly coupled quantum discrete Liouville theory, I: Algebraic approach and duality, Comm. Math. Phys. 219 (2001) 199-219 MR1828812

[9] S Gukov, Three-dimensional quantum gravity, Chern-Simons theory, and the Apolynomial, Comm. Math. Phys. 255 (2005) 577-627 MR2134725

[10] S Gukov, H Murakami, SL(2, C) Chern-Simons theory and the asymptotic behavior of the colored Jones polynomial, Lett. Math. Phys. 86 (2008) 79-98 MR2465747

[11] R M Kashaev, Quantum dilogarithm as a 6j-symbol, Modern Phys. Lett. A 9 (1994) 3757-3768 MR1317945

[12] R M Kashaev, A link invariant from quantum dilogarithm, Modern Phys. Lett. A 10 (1995) 1409-1418 MR1341338

[13] R M Kashaev, The hyperbolic volume of knots from the quantum dilogarithm, Lett. Math. Phys. 39 (1997) 269-275 MR1434238

[14] H Murakami, The coloured Jones polynomial, the Chern-Simons invariant and the Reidemeister torsion of the figure-eight knot, J. Topol. 6 (2013) 193-216 MR3029425 
[15] H Murakami, J Murakami, The colored Jones polynomials and the simplicial volume of a knot, Acta Math. 186 (2001) 85-104 MR1828373

[16] H Murakami, J Murakami, M Okamoto, T Takata, Y Yokota, Kashaev's conjecture and the Chern-Simons invariants of knots and links, Experiment. Math. 11 (2002) 427435 MR1959752

[17] T Ohtsuki, On the asymptotic expansion of the Kashaev invariant of the $5_{2}$ knot, preprint Available at http://www.kurims.kyoto-u.ac.jp/ tomotada/paper/ $\mathrm{ki} 52 \cdot \mathrm{pdf}$

[18] T Ohtsuki, On the asymptotic expansion of the Kashaev invariant of the hyperbolic knots with 7 crossings, in preparation

[19] T Ohtsuki, R Riley, M Sakuma, Epimorphisms between 2-bridge link groups, from: "The Zieschang Gedenkschrift", (M Boileau, M Scharlemann, R Weidmann, editors), Geom. Topol. Monogr. 14 (2008) 417-450 MR2484712

[20] T Ohtsuki, Y Yokota, On the asymptotic expansion of the Kashaev invariant of the knots with 6 crossings, preprint

[21] R Riley, Parabolic representations of knot groups, I, Proc. London Math. Soc. 24 (1972) 217-242 MR0300267

[22] R Riley, Nonabelian representations of 2-bridge knot groups, Quart. J. Math. Oxford Ser. 35 (1984) 191-208 MR745421

[23] R Riley, Algebra for Heckoid groups, Trans. Amer. Math. Soc. 334 (1992) 389-409 MR1107029

[24] M Sakuma, J Weeks, Examples of canonical decompositions of hyperbolic link complements, Japan. J. Math. 21 (1995) 393-439 MR1364387

[25] D P Thurston, Hyperbolic volume and the Jones polynomial Available at http:// pages.iu.edu/ dpthurst/speaking/Grenoble.pdf

[26] A T Tran, Twisted Alexander polynomials with the adjoint action for some classes of knots, J. Knot Theory Ramifications 23 (2014) MR3277960

[27] V G Turaev, Reidemeister torsion in knot theory, Uspekhi Mat. Nauk 41 (1986) 97-147, 240 MR832411 In Russian; translated in Russian Math. Surveys 41 (1986) 119-182

[28] E Witten, Quantum field theory and the Jones polynomial, Comm. Math. Phys. 121 (1989) 351-399 MR990772

[29] E Witten, Analytic continuation of Chern-Simons theory, from: "Chern-Simons gauge theory: 20 years after”, (J E Andersen, H U Boden, A Hahn, B Himpel, editors), AMS/IP Stud. Adv. Math. 50, Amer. Math. Soc. (2011) 347-446 MR2809462

[30] S L Woronowicz, Quantum exponential function, Rev. Math. Phys. 12 (2000) 873-920 MR1770545

[31] Y Yokota, On the volume conjecture for hyperbolic knots arXiv:math.QA/0009165 
[32] Y Yokota, From the Jones polynomial to the A-polynomial of hyperbolic knots, from: "Proceedings of the Winter Workshop of Topology/Workshop of Topology and Computer", Interdiscip. Inform. Sci. 9 (2003) 11-21 MR2023102

[33] D Zagier, Quantum modular forms, from: "Quanta of maths", (E Blanchard, D Ellwood, M Khalkhali, M Marcolli, S Popa, editors), Clay Math. Proc. 11, Amer. Math. Soc. (2010) 659-675 MR2757599

Research Institute for Mathematical Sciences, Kyoto University

Sakyo-ku, Kyoto, 606-8502, Japan

Faculty of Mathematics, Kyushu University

Fukuoka, 819-0395, Japan

tomotada@kurims.kyoto-u.ac.jp, ttakata@math.kyushu-u.ac.jp

http://www.kurims.kyoto-u.ac.jp/ tomotada/

Proposed: Shigeyuki Morita

Received: 4 August 2013

Seconded: Vaughan Jones, Jean-Pierre Otal

Revised: 8 April 2014 